Aus der Abteilung Allgemeinmedizin

(Prof. Dr. med. M. M. Kochen, MPH, FRCGP)

im Zentrum Innere Medizin

der Medizinischen Fakultät der Universität Göttingen

\title{
Allgemeinärztliche Beurteilungen und Einstellungen zur Sterbehilfe
}

Eine nationale Erhebung

\author{
INAUGURAL - DISSERTATION \\ zur Erlangung des Doktorgrades \\ der Medizinischen Fakultät \\ der Georg-August-Universität zu Göttingen \\ vorgelegt von \\ Anja Harfst \\ aus \\ Rüsselsheim
}

Göttingen 2004 
I. Berichterstatter: Prof. Dr. med. M. M. Kochen, MPH, FRCGP

II. Berichterstatterlin: Prof. Dr. med. D. Kettler

III. Berichterstatterlin:

Tag der mündlichen Prüfung: 7. Juni 2005 


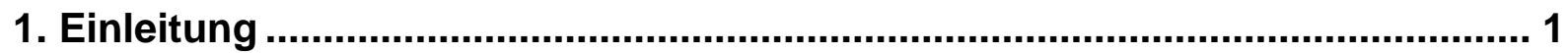

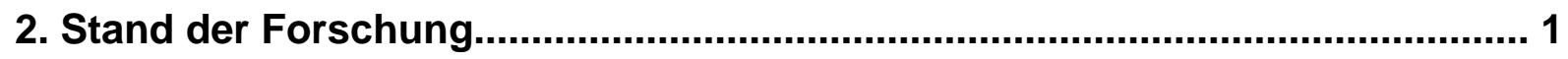

2.1 Internationale Untersuchungen zur Haltung von Ärzten zur Sterbehilfe .................................... 1

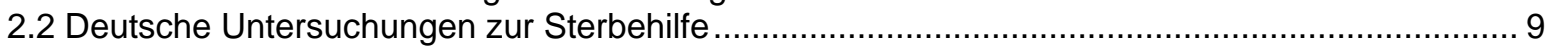

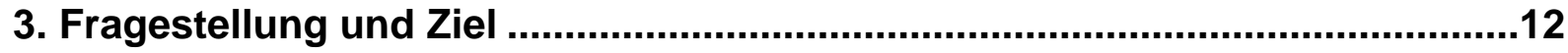

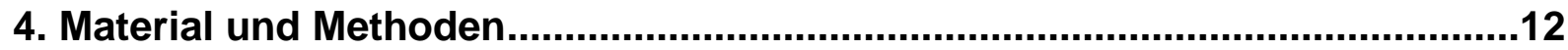

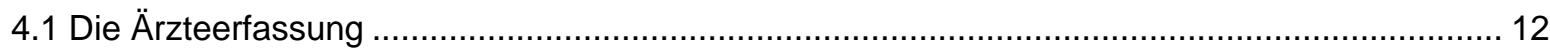

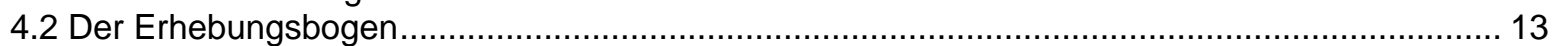

4.3 Pilotstudie und Versenden der Fragebögen ................................................................. 15

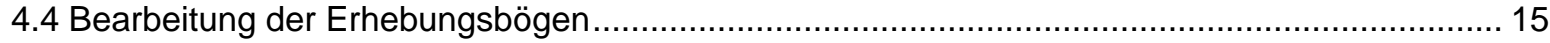

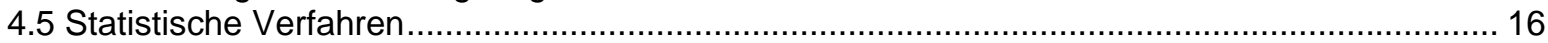

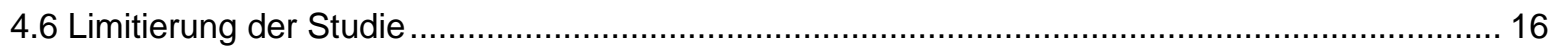

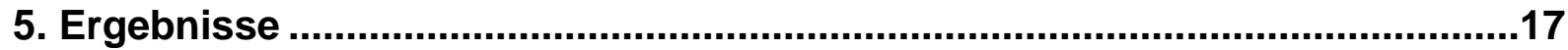

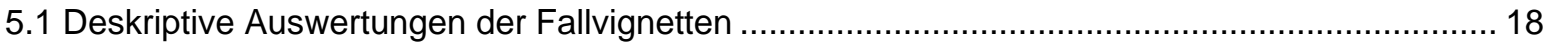

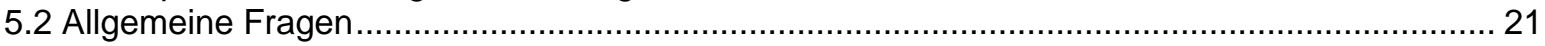

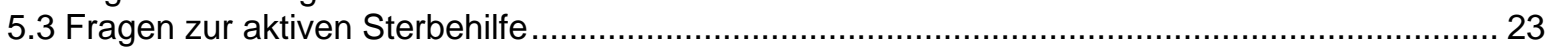

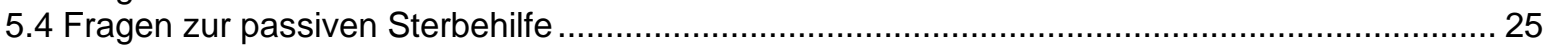

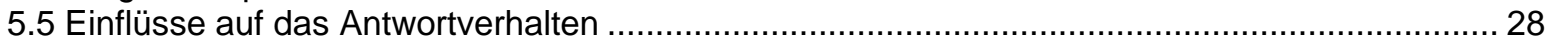

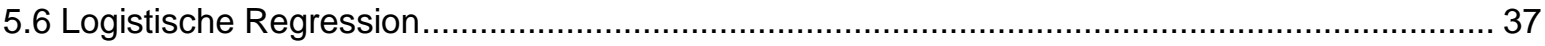

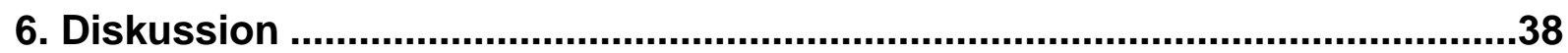

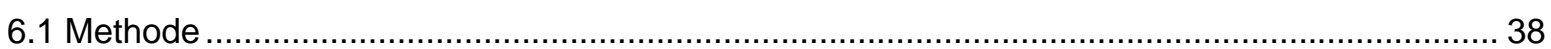

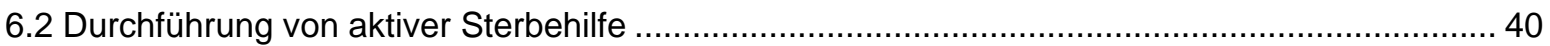

6.3 Gründe für das Durchführen von aktiver Sterbehilfe ....................................................... 41

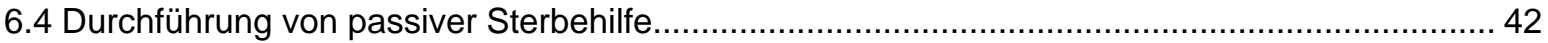

6.5 Thematisierung von Sterbehilfe in der medizinischen Ausbildung ......................................... 44

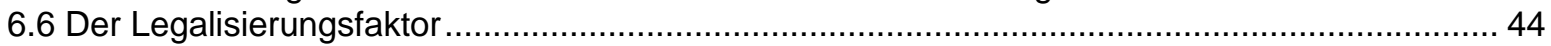

6.7 Beziehung zwischen Euthanasie im sog. "Dritten Reich" und ärztlicher Sterbehilfe ................... 46

6.8 Einflussfaktoren bezüglich Einstellungen zur Sterbehilfe ................................................ 47

6.9 Vergleich mit den Niederlanden bei der Nachfrage nach Sterbehilfe ...................................... 48

6.10 Einstellung zur Sterbehilfe im internationalen Vergleich ................................................ 49

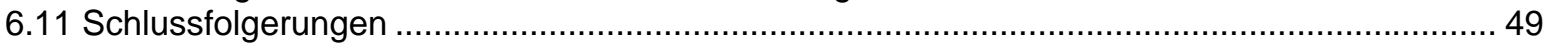

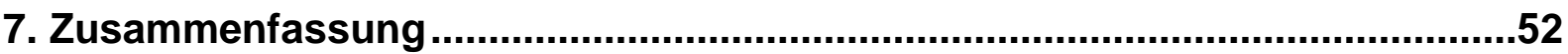

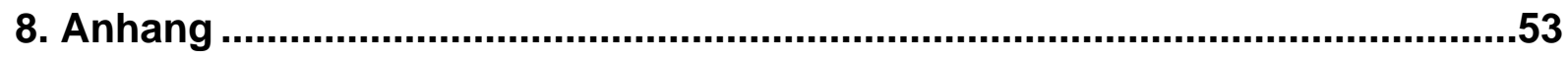

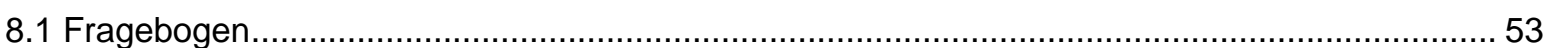

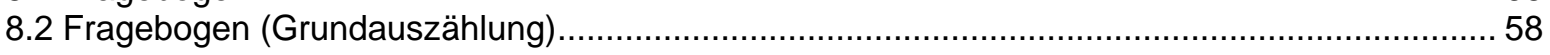

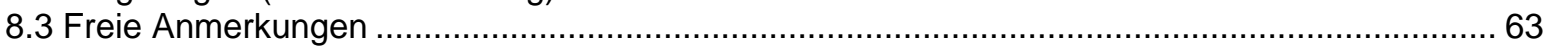

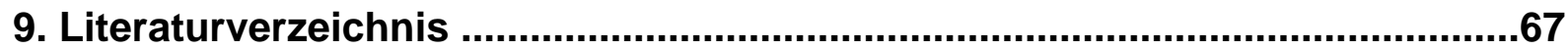

\section{Anmerkung}

Der besseren Lesbarkeit wegen wird im Text die männliche Sprachform gewählt. Selbstverständlich sind beide Geschlechter gemeint. 


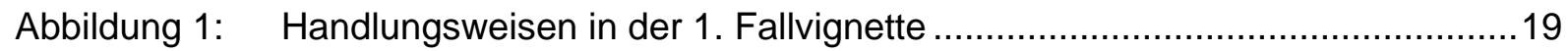

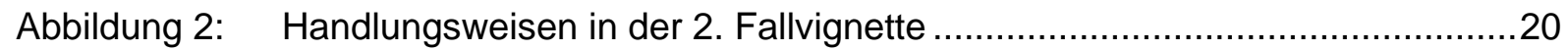

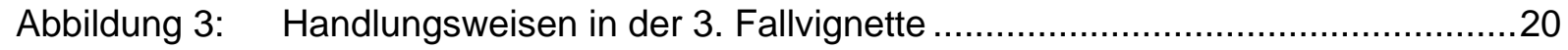

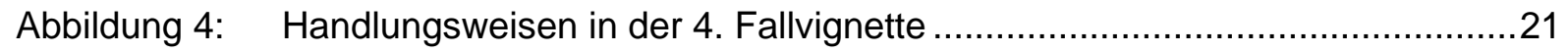

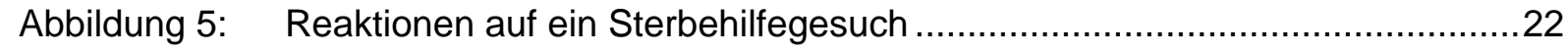

Abbildung 6: Erhaltene Nachfragen nach aktiver Sterbehilfe in den letzten zehn Jahren .23

Abbildung 7: Beweggründe für die Durchführung von aktiver Sterbehilfe ........................23

Abbildung 8: Beweggründe gegen die Durchführung von aktiver Sterbehilfe....................24

Abbildung 9: Gründe, die die Durchführung aktiver Sterbehilfe erleichtern würden ..........25

Abbildung 10: Nachfragen nach passiver Sterbehilfe in den letzten zehn Jahren..............26

Abbildung 11: Beweggründe zur Durchführung passiver Sterbehilfe ................................ 26

Abbildung 12: Beweggründe gegen die Durchführung der passiven Sterbehilfe................27

Abbildung 13: Gründe, die die Durchführung von passiver Sterbehilfe erleichtern würden.28

Abbildung 14: Zustimmung zur aktiven und passiven Sterbehilfe in Abhängigkeit von der Konfession

Abbildung 15: Bereitschaft zur Durchführung von aktiver und passiver Sterbehilfe in Abhängigkeit von der Größe der Praxis.... .30

Abbildung 16: Ablehnung der Durchführung von Sterbehilfe in Abhängigkeit vom Jahr der Approbation

Abbildung 17: Akzeptanz der aktiven Sterbehilfe in Abhängigkeit von erhaltenen Nachfragen

Abbildung 18: Bereitschaft aktive bzw. passive Sterbehilfe durchzuführen in Abhängigkeit von erhaltenen Nachfragen

Abbildung 19: Akzeptanz von aktiver und passiver Sterbehilfe in Abhängigkeit von einer (mit)erlebten lebensbedrohlichen Krankheit

Abbildung 20: Bereitschaft zur Durchführung aktiver und passiver Sterbehilfe in Abhängigkeit von einer (mit)erlebten lebensbedrohlichen Krankheit 33

Abbildung 21: Akzeptanz der aktiven und passiven Sterbehilfe bei Ärzten, die einen Vergleich zwischen der Euthanasie im sog. "Dritten Reich" und der heutigen Sterbehilfe für gerechtfertigt halten.

Abbildung 22: Bereitschaft zur Durchführung aktiver und passiver Sterbehilfe in Abhängigkeit individueller gesetzlicher Voraussetzungen ..... 35

Abbildung 23: Einfluss der Beschäftigung mit den Themen "Sterben" und "Sterbehilfe" während der Ausbildung auf die Akzeptanz aktiver und passiver Sterbehilfe .. 
Abbildung 24: Verhalten von Ärzten, die gegenwärtig die Durchführung von Sterbehilfe ablehnen, im Falle einer Legalisierung

Abbildung 25: Bereitschaft zur Durchführung aktiver bzw. passiver Sterbehilfe in der zur Zeit geltenden Rechtslage bezogen auf die einzelnen Fallvignetten.

Tabelle 1: $\quad$ Untersuchungen von Allgemeinärzten zur Sterbehilfe 3

Tabelle 2: Deutsche Untersuchungen zur Sterbehilfe 6

Tabelle 3: $\quad$ Charakteristika der teilnehmenden Ärzte 17

Tabelle 4: $\quad$ Akzeptanz aktiver und passiver Sterbehilfe bei Ärzten mit und ohne Kinder. 29

Tabelle 5: Bereitschaft zur Durchführung von aktiver und passiver Sterbehilfe in Abhängigkeit einer erfolgten onkologischen Tätigkeit. 29

Tabelle 6: $\quad$ Akzeptanz der aktiven und passiven Sterbehilfe in Abhängigkeit einer bereits erlebten geriatrischen Tätigkeit. 30

Tabelle 7: Bereitschaft aktive oder passive Sterbehilfe durchzuführen in Abhängigkeit von der Größe des Ortes der Praxis 


\section{Einleitung}

Das 2001 in den Niederlanden in Kraft getretene „Gesetz zur Überprüfung der Lebensbeendigung auf Verlangen und der Hilfe bei der Selbsttötung“ hat mit der teilweisen Legalisierung von Sterbehilfe international für Aufsehen gesorgt. Obgleich weltweit in einer Vielzahl von Arbeiten die Akzeptanz und Position von Ärzten zur Sterbehilfe untersucht ist, stellt sie in Deutschland ein noch weitgehend unbearbeitetes Gebiet dar. Die Ursachen hierfür liegen im Unklaren, obwohl seit Mitte der 80er Jahre die Verbrechen der „Euthanasie“ im sog. „Dritten Reich" systematisch aufgearbeitet und in einer Vielzahl von Beiträgen Stellungnahmen zur Sterbehilfe verfasst wurden.

In mehreren Veröffentlichungen wurden zudem in einer Debatte des letzten Jahrzehnts ethische, moralische und philosophische Stellungnahmen zur Sterbehilfe dargestellt (Hegselmann und Merkel 1991). In einer weithin diskutierten Stellungnahme veröffentlichte in diesem Zusammenhang auch die Bundesärztekammer "Grundsätze zur ärztlichen Sterbebegleitung" (Bundesärztekammer 1998). Einerseits wurde hier festgehalten, dass aktive Sterbehilfe auch auf Verlangen des Patienten unzulässig sei und dem ärztlichen Ethos widerspräche. Auf der anderen Seite wurde allerdings auch festgestellt, dass "die ärztliche Verpflichtung zur Lebenserhaltung (...) nicht unter allen Umständen" bestünde und "Begrenzung geboten sein kann". Auch dürfe in bestimmten Situationen eine "möglicherweise unvermeidbare Lebensverkürzung" durch Maßnahmen zur Linderung eines Leidens hingenommen werden (Bundesärztekammer 1998, A-2366).

Ungeachtet dieser klaren Positionen ist auffällig, dass gerade im Vergleich zum Ausland in Deutschland nur sehr wenige Untersuchungen durchgeführt wurden, die sich mit der Position deutscher Ärzte zur Sterbehilfe befassen.

\section{Stand der Forschung}

\subsection{Internationale Untersuchungen zur Haltung von Ärzten zur Sterbehilfe}

Es existieren viele Veröffentlichungen zum Thema Sterbehilfe. Eine im Januar 2002 durchgeführte Literaturrecherche über Medline erbrachte alleine unter dem Suchbegriff „euthanasia“ 3098 Treffer für die letzten 10 Jahre aus den verschiedensten Ländern. Untersuchungen zu verwandten Themenbereichen haben gezeigt, dass die Einstellungen zur ärztlichen Tätigkeit am Lebensende von Patienten in Abhängigkeit vom soziokulturellen und nationalen Kontext variieren (Molloy et al. 1991). Ungeachtet dessen existieren keine länderübergreifenden Erhebungen zu Einstellungen von Ärzten zur Sterbehilfe. Nur eine geringe Zahl an Studien untersucht explizit die Haltung von Allgemeinärzten. 
Ausgehend von der Vielzahl unterschiedlichster Untersuchungen im Ausland stellen die vorgetragenen Ergebnisse der nationalen Studien nur eine Auswahl dar. Die vorliegende Arbeit beschränkt sich auf Erhebungen, bei denen die Position von Allgemeinärzten gesondert berücksichtigt wurde, die Befragung nicht nur auf Fallbeispielen basierte und eine Vollerhebung oder Randomisation der Ärzte erfolgte. Eine Übersicht der untersuchten Studien gibt Tabelle 1. 
Tabelle 1: Untersuchungen von Allgemeinärzten (Allg. Ärzte) zur Sterbehilfe ${ }^{1}$

Allgemeinärztliche Positionen, wo gesondert gekennzeichnet $\left(^{*}\right)$.

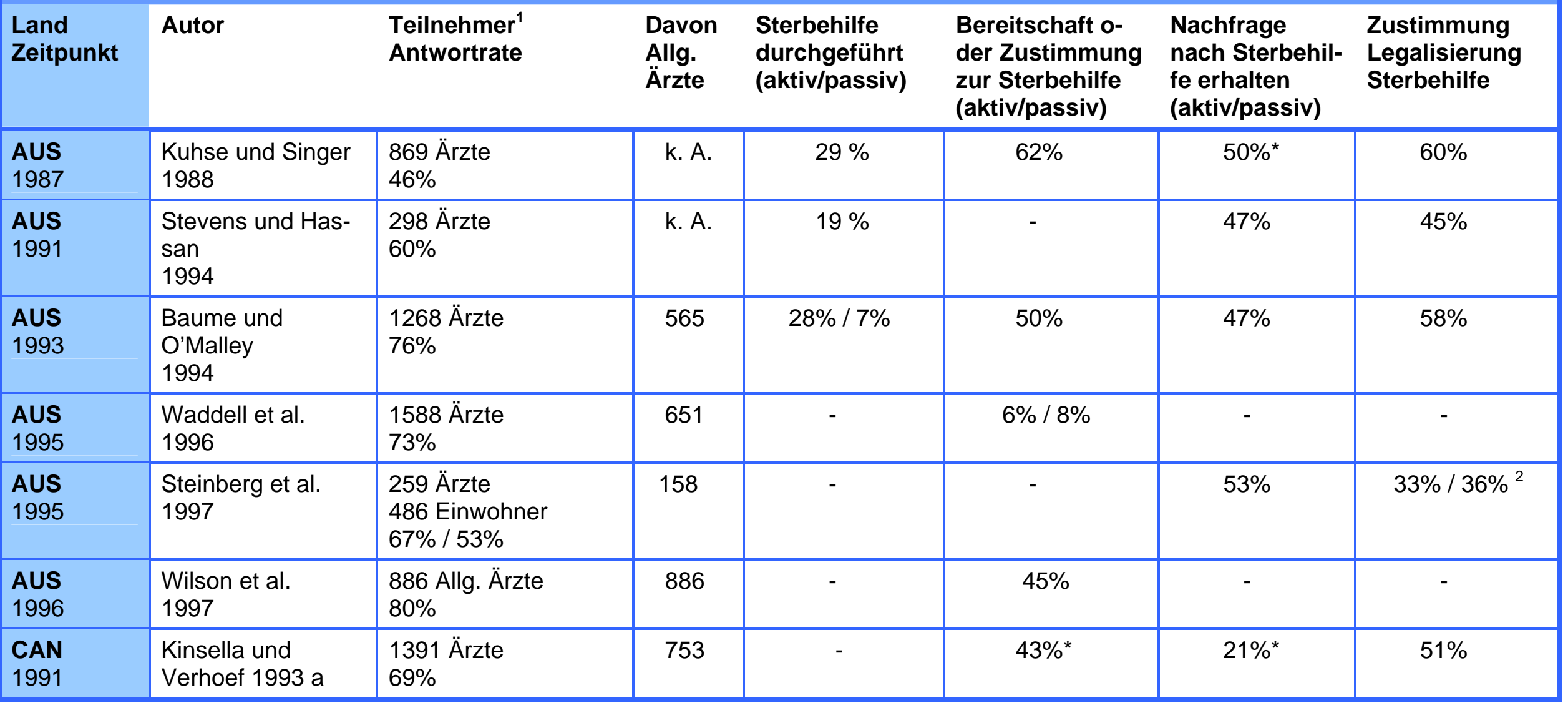

\footnotetext{
${ }^{1}$ Angabe der verwertbaren Antworten

${ }^{2}$ Aktive / passive Sterbehilfe
} 


\begin{tabular}{|c|c|c|c|c|c|c|c|}
\hline $\begin{array}{l}\text { CAN } \\
1994\end{array}$ & $\begin{array}{l}\text { Verhoef und Kin- } \\
\text { sella } \\
1996\end{array}$ & $\begin{array}{l}866 \text { Ärzte } \\
77 \%\end{array}$ & 471 & - & $42 \%$ & $19 \%$ & $37 \%$ \\
\hline $\begin{array}{l}\text { DK } \\
1995\end{array}$ & $\begin{array}{l}\text { Folker et al. } \\
1996^{3}\end{array}$ & $\begin{array}{l}314 \text { Ärzte } \\
64 \%\end{array}$ & 141 & $5 \% / 12-80 \%$ & $10 \% / 10 \%$ & $30 \% / 22-74 \%$ & $29 \% / 13-95 \%$ \\
\hline $\begin{array}{l}F \\
2002\end{array}$ & $\begin{array}{l}\text { Peretti-Watel et al. } \\
2003\end{array}$ & $\begin{array}{l}\text { 502/1004 Allg. Ärzte } \\
50 \%{ }^{4}\end{array}$ & 502 & - & - & - & $44,8 \%$ * \\
\hline $\begin{array}{l}\text { GB } \\
1980\end{array}$ & $\begin{array}{l}\text { Keane et al. } \\
1983\end{array}$ & $\begin{array}{l}\text { 313/950 Allg. Ärzte } \\
\text { 33\% }\end{array}$ & 313 & - & - & $3 \%$ & $12 \%$ \\
\hline $\begin{array}{l}\text { GB } \\
1992-1993\end{array}$ & $\begin{array}{l}\text { Ward BJ und Tate } \\
1994\end{array}$ & $\begin{array}{l}203 \text { Chefärzte } \\
221 \text { Allg. Ärzte } \\
73,6 \%\end{array}$ & 221 & $32 \% *$ & $88 \% *$ & $64 \% *$ & $48 \%$ * \\
\hline $\begin{array}{l}\text { GB } \\
\text { k. A. }\end{array}$ & $\begin{array}{l}\text { McGlade et. al. } \\
2000\end{array}$ & 1053 Allg. Ärzte & 1053 & $10 \% / 55 \%{ }^{5}$ & $49 \%$ & $30 \%$ & - \\
\hline $\begin{array}{l}\mathbf{N} \\
1993\end{array}$ & $\begin{array}{l}\text { Forde et al. } \\
1997\end{array}$ & $\begin{array}{l}980 \text { Ärzte } \\
66 \%\end{array}$ & 247 & $6 \%$ & $18 \%^{6}$ & - & - \\
\hline $\begin{array}{l}\text { NL } \\
1990-1991\end{array}$ & $\begin{array}{l}\text { Van der Maas et } \\
\text { al. } \\
1991\end{array}$ & $\begin{array}{l}405 \text { Ärzte } \\
100 \%^{7}\end{array}$ & 152 & $62 \% *$ & $90 \% *$ & - & - \\
\hline $\begin{array}{l}\text { NL } \\
\text { k. A. }\end{array}$ & $\begin{array}{l}\text { Onwuteaka et al. } \\
1995\end{array}$ & $\begin{array}{l}686 \text { Allg. Ärzte } \\
613 \text { Ärzte einer } \\
\text { Privatklinik } \\
67 \% / 86 \%\end{array}$ & 686 & - & - & - & $23 \%$ \\
\hline
\end{tabular}

\footnotetext{
${ }_{4}^{3}$ Die differenten prozentualen Angabe dieser Untersuchung basieren auf unterschiedlichen Zustimmungsraten zu verschiedenen Fallvignetten.

${ }^{4}$ Telephonische Befragung

${ }^{5}$ Bezug auf die 107 Allgemeinärzte, die zu dieser Frage Stellung bezogen

${ }^{6}$ Bei präterminalen Patienten

${ }^{7}$ Interviews
} 


\begin{tabular}{|c|c|c|c|c|c|c|c|}
\hline $\begin{array}{l}\text { NL } \\
1986-1989\end{array}$ & $\begin{array}{l}\text { Verhoef und van } \\
\text { der Waal. } \\
1997\end{array}$ & $\begin{array}{l}405 \text { Allg. Ärzte } \\
67 \%\end{array}$ & 405 & $24 \%$ & - & $73 \%$ & - \\
\hline $\begin{array}{l}\text { USA } \\
1991\end{array}$ & $\begin{array}{l}\text { Shapiro et al. } \\
1994\end{array}$ & $\begin{array}{l}740 \text { Ärzte } \\
33 \%\end{array}$ & 402 & $2,2 \% /-$ & $54,3 \% *$ & $48,2 \% /-$ & - \\
\hline $\begin{array}{l}\text { USA } \\
1991\end{array}$ & $\begin{array}{l}\text { Duberstein et al. } \\
1995\end{array}$ & $\begin{array}{l}114 \text { Ärzte } \\
61 \%\end{array}$ & 31 & - & $-/ 51 \% *$ & - & $31 \%$ \\
\hline $\begin{array}{l}\text { USA } \\
1992\end{array}$ & $\begin{array}{l}\text { Cohen et al. } \\
1994\end{array}$ & $\begin{array}{l}938 \text { Ärzte } \\
69 \%\end{array}$ & 188 & - & $42 \% / 50 \%$ & - & $53 \%$ \\
\hline $\begin{array}{l}\text { USA } \\
1994-1995\end{array}$ & $\begin{array}{l}\text { Bachmann et al. } \\
1996\end{array}$ & $\begin{array}{l}1119 \text { Ärzte } \\
998 \text { Einwohner } \\
74 \% / 76 \%\end{array}$ & 632 & - & $39 \% * /-$ & - & $54 \% *$ \\
\hline $\begin{array}{l}\text { USA } \\
1994-1995\end{array}$ & $\begin{array}{l}\text { Back et al. } \\
1996\end{array}$ & $\begin{array}{l}828 \text { Ärzte } \\
57 \%\end{array}$ & 258 & $24 \% / 24 \%{ }^{8}$ & - & $-/ 26 \%$ & - \\
\hline $\begin{array}{l}\text { USA } \\
1995\end{array}$ & $\begin{array}{l}\text { Lee et al. } \\
1996\end{array}$ & $\begin{array}{l}2761 \text { Ärzte } \\
70 \%\end{array}$ & 754 & $-/ 7 \%$ & $-/ 46 \%$ & $-/ 21 \%$ & $60 \%$ \\
\hline $\begin{array}{l}\text { USA } \\
1996\end{array}$ & Meier et al. 1998 & $\begin{array}{l}275 \text { Onkologen; } 71 \% \\
1902 \text { Nicht- } \\
\text { Onkologen; } 61 \%\end{array}$ & 197 & $4 \% * / 2 \% *$ & $7 \% \%^{*} / 10 \% *$ & $8 \% * / 15 \% *$ & - \\
\hline $\begin{array}{l}\text { YUG } \\
1998\end{array}$ & $\begin{array}{l}\text { Radulovic und Mo- } \\
\text { jsilovic } \\
1998\end{array}$ & $\begin{array}{l}61 \text { Ärzte, } 31 \text { Medizin- } \\
\text { studenten, } 31 \\
\text { Rechtsanwälte } \\
\text { - }\end{array}$ & 31 & - & $11 \%$ * & - & $30 \% *$ \\
\hline
\end{tabular}

\footnotetext{
${ }^{8}$ In Bezug auf 147 Ärzte
} 


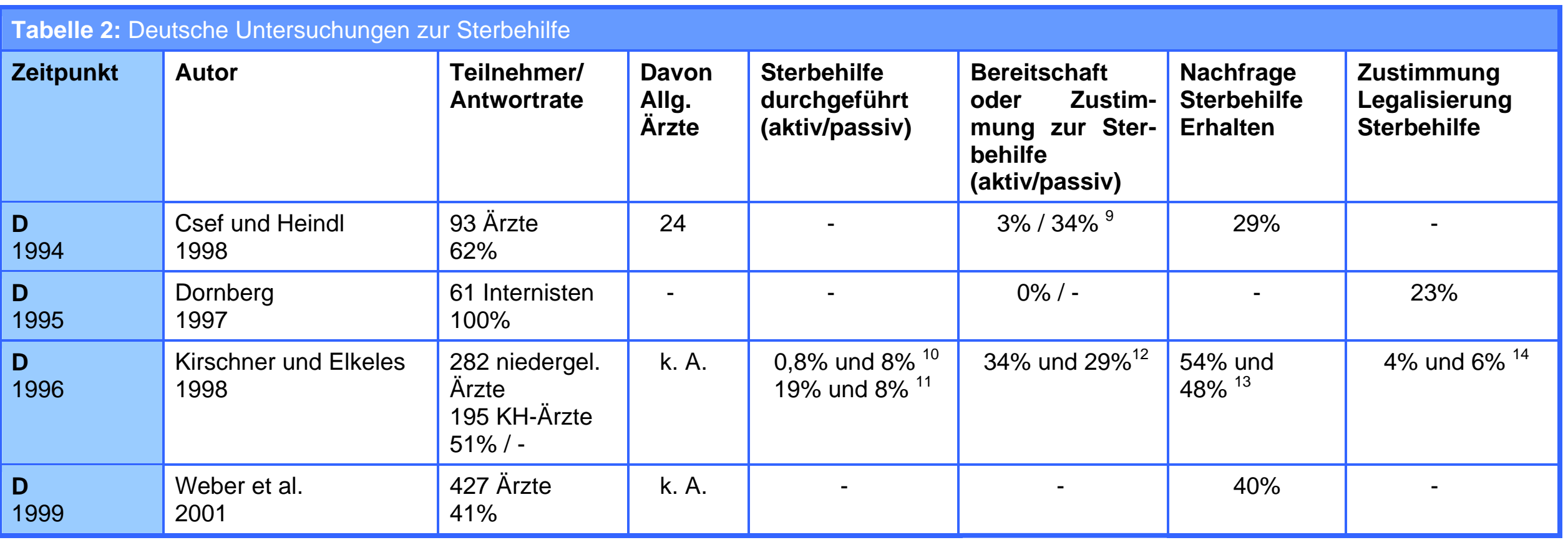

\footnotetext{
${ }^{9} 50 \%$ Zustimmung zur Sterbehilfe unter bestimmten Bedingungen

${ }^{10}$ Aktive Sterbehilfe Krankenhaus- und niedergelassene Ärzte

${ }^{11}$ Indirekte Sterbehilfe Krankenhaus- und niedergelassene Ärzte

${ }^{12}$ Krankenhaus- und niedergelassene Ärzte. „Akzeptanz“ bei 54\% Krankenhausärzte und $45 \%$ niedergelassene Ärzte

${ }^{13}$ Sterbehilfe Krankenhaus- und niedergelassene Ärzte

${ }^{14}$ Sterbehilfe Krankenhaus- und niedergelassene Ärzte
} 


\subsubsection{Europa}

Die meisten europäischen Befragungen zur Sterbehilfe wurden in den Niederlanden und in Großbritannien durchgeführt. Bereits 1980 konnten Keane et al. in einer Erhebung unter britischen Allgemeinärzten eine Zustimmung von $12 \%$ für die Legalisierung von Sterbehilfe feststellen (Keane et al. 1983). 1992 - 1994 führten Ward BJ und Tate eine Erhebung unter 221 Allgemeinärzten und 203 Chefärzten durch. 64\% der Allgemeinärzte (entspricht 76\% aller Ärzte) gaben an, schon Nachfragen nach Sterbehilfe erhalten zu haben. Von den Allgemeinärzten hatten $32 \%$ bereits aktive Sterbehilfe durchgeführt und 88\% äußerten die Bereitschaft zu passiver Sterbehilfe (Ward BJ und Tate 1994). In einer aktuellen Untersuchung unter Allgemeinärzten in Nordirland hielten $70 \%$ der Teilnehmer passive Sterbehilfe für moralisch und ethisch akzeptabel. 49\% waren zur Durchführung passiver Sterbehilfe bereit. Aktive Sterbehilfe und Beihilfe zur Selbsttötung wurde hingegen von der Mehrheit abgelehnt (Mc Glade et al. 2000).

In der Sterbehilfe nehmen die Niederlande eine Sonderstellung in Europa ein. Schon vor der Legalisierung wurde die Diskussion mit großer Offenheit geführt und die meisten europäischen Studien sind in Holland angesiedelt. In einer mehrteiligen Studie führten van der Maas und Mitarbeiter an, dass 62\% Teilnehmer aktive Sterbehilfe praktiziert oder Beihilfe zur Selbsttötung geleistet hatten. Weitere $28 \%$ erklärten sich unter gewissen Bedingungen hierzu bereit. Lediglich $3 \%$ der Hausärzte wollten keinesfalls im Sinne einer Sterbehilfe tätig werden (van der Maas et al. 1991). In einer weiteren Erhebung von Onwuteaka- Philipsen et al. wurde zwar nur von 23\% der teilnehmenden Ärzte die Legalisierung gefordert; aber auch hier äußerten sich lediglich $10 \%$ ablehnend zur Sterbehilfe. Diese Position wurde überwiegend von Älteren, religiös Aktiven sowie Ärzten, die noch nie Sterbehilfe durchgeführt hatten, vertreten (Onwuteaka- Philipsen et al. 1995). Verhoef und van der Waal führten von 1986 - 1987 eine Befragung nur unter Hausärzten durch. Ein Viertel der Teilnehmer gab an, bereits Sterbehilfe durchgeführt zu haben. 73\% hatten bereits Nachfragen hierzu erhalten, wobei aktive Sterbehilfe 3,5mal häufiger erbeten wurde als Beihilfe zur Selbsttötung (Verhoef und van der Waal 1997).

Weitere europäische Beiträge zur Sterbehilfe stammen aus den skandinavischen Ländern. In einer 1995 durchgeführten Untersuchung bei dänischen Ärzten wurde Sterbehilfe nur von einer Minderheit befürwortet. Ungeachtet einer moralischen Akzeptanz bei 1/3 der Teilnehmer war aktive Sterbehilfe nur von $5 \%$ durchgeführt worden, allerdings votierten $29 \%$ für eine Legalisierung von Sterbehilfe (Folker et al. 1996). Ähnlich wurde bei einer Erhebung unter norwegischen Ärzten aus dem Jahr 1993 nur von 6\% der Ärzte berichtet, Sterbehilfe geleistet zu haben. Hingegen schilderten $76 \%$, dass sie Patienten weiterbehandelt hätten, obgleich sie einen Behandlungsabbruch befürwortet hätten. Die Allgemeinärzte der Untersuchung äußerten im Vergleich zu anderen Arztgruppen eine negativere Einstellung zur Sterbehilfe (Forde et al. 1997). 
In der 2003 erschienenen Studie von Peretti- Watel und Mitarbeitern aus Frankreich hingegen votierten $45 \%$ der befragten Allgemeinärzte für eine Legalisierung von Sterbehilfe (Peretti- Watel et al. 2003).

Aus dem südeuropäischen Raum existiert nur eine kleine Untersuchung an 123 Ärzten aus Belgrad, die sich mit der Haltung von Ärzten zur Sterbehilfe auseinandersetzt. Von den Allgemeinärzten äußerten 11\% Bereitschaft zur Sterbehilfe und 30\% traten für die Legalisierung von Sterbehilfe ein (Radulovic und Mojsilovic 1998).

\subsubsection{Australien}

Wie die Niederlande nimmt Australien eine Sonderstellung ein. 1995 wurde der „Northern Territory Rights of the Terminally III Act“ verabschiedet, mit dem die ärztliche Durchführung von Sterbehilfe legalisiert wurde. Die von Kuhse und Singer 1987 durchgeführte Erhebung gehört zu einer der meistzitierten Studien, die später auch wesentliche Gesetzesmaßnahmen begründete. 1/3 der befragten Ärzte hatte bereits Sterbehilfe durchgeführt und 62\% äußerten eine generelle Zustimmung. Die Hälfte der Allgemeinärzte hatte Nachfragen nach Sterbehilfe erhalten und war häufiger zur Durchführung bereit (Kuhse und Singer 1988). In einer weiteren australischen Studie wurde diese Quote bestätigt: 1991 äußerten 59\% der Ärzte Zustimmung zur Sterbehilfe. 28\% hatten bereits Sterbehilfe und 7\% Beihilfe zur Selbsttötung geleistet. Bei der Mehrheit bestand der Wunsch nach Legalisierung von Sterbehilfe (Baume und O' Malley 1994). Bis auf eine Ausnahme (Waddell et al. 1996) stützen weitere Studien diese Position mit ähnlich hohen Quoten (Steinberg et al. 1997; Stevens und Hassan 1994). Auch in der 1997 von Wilson durchgeführten Erhebung unter australischen Hausärzten äußerten 45\% der Teilnehmer Zustimmung zu der Option Sterbehilfe als Teil ärztlicher Versorgung (Wilson et al. 1997).

\subsubsection{Nordamerika}

Auch in den USA wurde in der Folge von Diskussionen um Sterbehilfe 1994 in Oregon mit knapper Mehrheit der „Death with Dignity Act“ verabschiedet. Mit diesem Gesetz wurde die Beihilfe zur Selbsttötung - nicht aber aktive Sterbehilfe - bei terminal Kranken straffrei gestellt. Allerdings wurde das Gesetz 1995 durch ein Bundesgericht für ungültig erklärt und aufgehoben. Während dieser Zeit erschien eine ganze Reihe von Erhebungen zu Einstellungen von Ärzten zur Sterbehilfe (Emanuel et al. 1996, S. 1805) .

Eine 1991 in Wisconsin durchgeführte Untersuchung ergab eine Zustimmung zur Sterbehilfe bei 34\% der Allgemeinärzte, die häufiger zur Durchführung von Sterbehilfe bereit waren als andere Ärztegruppen (Shapiro et al. 1994). Eine im nächsten Jahr in Washington State durchgeführte Erhebung erbrachte ebenfalls eine Zustimmung zur Sterbehilfe bei $42 \%$ der Befragten. Mehr als die Hälfte war der Meinung, dass Sterbehilfe in bestimmten Situationen legal sein sollte und 1/3 war 
bereit, selbst Sterbehilfe durchzuführen. Besondere Unterschiede zwischen den Arztgruppen ergaben sich insoweit, als Onkologen eher gegen und Psychiater eher für Sterbehilfe plädierten (Cohen et al. 1994). Eine ähnliche hohe Zustimmung von 52\% fand sich in einer 1993 in den Südstaaten durchgeführten Untersuchung (Dickinson et al. 1996). Die 1994 - 1995 durchgeführte Studie von Back et al. zeigte, dass Allgemeinärzte im Vergleich am häufigsten Nachfragen nach Sterbehilfe erhielten (Back et al. 1996). Eine weitere Erhebung im Staate New York unter „primary- care“Ärzten erbrachte eine Zustimmung für Beihilfe zur Selbsttötung bei 31\% der Befragten (Duberstein et al. 1995). Noch vor der Aufhebung des „Death with Dignity Act“ wurde 1995 in Oregon eine Befragung durchgeführt, in der $46 \%$ die Beihilfe zur Selbsttötung für ethisch vertretbar hielten (Lee et al. 1996). Meier et al. konnten in ihrer 1998 veröffentlichten Studie zeigen, dass 4\% bzw. 2\% der befragten Allgemeinärzte bereits aktive bzw. passive Sterbehilfe durchgeführt hatten (Meier et al. 1998).

Die in Kanada im Verlauf mehrerer Jahre durchgeführten Studien belegen, dass auch hier die Zustimmung zur Sterbehilfe vergleichsweise hoch ist. In den Untersuchungen von Kinsella und Verhoef wurden 1991 und 1994 Zustimmungsquoten zur Sterbehilfe von 43\% bzw. 42\% festgestellt, wobei der Wunsch nach Legalisierung und die Bereitschaft zur Durchführung aktiver Sterbehilfe fiel (von 29\% 1991 auf 15\% 1994)(Kinsella und Verhoef 1993 a; Kinsella und Verhoef 1993 b; Kinsella und Verhoef 1995; Kinsella und Verhoef 1996).

\subsection{Deutsche Untersuchungen zur Sterbehilfe}

Im Gegensatz zur internationalen Situation ist in Deutschland nur wenig zur Haltung von Ärzten in der Sterbehilfe bekannt.

In der ersten veröffentlichten Untersuchung aus dem Jahr 1997 von Martin Dornberg wurden Einstellungen deutscher Ärzte zur Sterbehilfe abgefragt. Es wurden hierzu Interviews mit Internisten in südbadischen Krankenhäusern durchgeführt. Bei allen befragten 61 Ärzten herrschte eine starke Ablehnung gegen aktive Sterbehilfe vor; die Durchführung entsprechender Maßnahmen wurde als „extrem selten“ eingestuft. Bei Vorliegen eines „mutmaßlichen Willens" durch Patiententestament bzw. Angehörige sprach sich eine Mehrheit (92\% bzw. 82\%) für den Behandlungsverzicht bei terminal erkrankten Patienten aus. Auch bei nicht bekanntem Patientenwillen befürworteten $69 \%$ der Befragten die Beendigung medizinischer Maßnahmen bei terminal Kranken und 69\% bei „nicht terminal Erkrankten mit schlechter Prognose“. 23\% äußerten den Wunsch nach einer rechtlichen Regelung von aktiver und passiver Sterbehilfe (Dornberg 1997, S. 99). In dieser Dissertation wurde besonders auf die Problematik des "Grauzonenbereichs“ aufmerksam gemacht, nämlich auf Schwierigkeiten bei der exakten Abgrenzung der Begriffe aktive vs. indirekte vs. passive Sterbehilfe, sowie auf die „Dammbruchproblematik“ und auf das Problem des „Handelns unter Unsicherheit“ und dessen Regulierbarkeit (z.B. Patiententestament) (Dornberg 1997, S. 99). Dass bei den meis- 
ten Ärzten Unklarheit über den juristischen Tatbestand von aktiver oder passiver Sterbehilfe herrscht, kommt weiter hinzu. Eingeschränkt ist diese Studie in ihrer generellen Aussagekraft durch die selektive Auswahl südbadischer internistischer Krankenhausärzte.

Eine weitere deutsche Untersuchung von Kirschner und Elkeles entstand 1996 im Auftrag des Verlages Gruner und Jahr (Kirschner und Elkeles 1998). Nach einem Pretest an 50 Ärzten mittels "Schneeballverfahren" wurden bundesweit 282 niedergelassene praktische Ärzte, Allgemeinärzte und Internisten und 191 Krankenhausärzte verschiedener Fachrichtungen über Stichproben ausgewählt. Diese wurden entweder schriftlich, telefonisch oder mündlich zur Sterbehilfe befragt. 48\% der niedergelassenen Ärzte hatten schon Bitten um aktive Sterbehilfe erhalten. 11\% hatten bereits Fälle von Sterbehilfe erlebt und $8 \%$ (bei Krankenhaus-Ärzten 0,8\%) hatten diese selbst durchgeführt. Im Durchschnitt hatten die Ärzte alle zwei Jahre eine Nachfrage nach aktiver Sterbehilfe erhalten; Onkologen doppelt so häufig. Wurde die aktive Sterbehilfe abgelehnt, waren als Hauptgründe hierfür genannt (Kirschner und Elkeles 1998, S. 251):

- „Frage der Berufsethik“

- „strafbares Handeln“

- "christlicher Glaube“

- „andere Möglichkeiten“ (passive oder indirekte Sterbehilfe)

Im Vergleich zur aktiven Sterbehilfe lag die Akzeptanz der indirekten Sterbehilfe deutlich höher: Indirekte Sterbehilfe wurde von $45 \%$ der niedergelassenen Ärzte nicht nur befürwortet, sondern war auch von 50\% durchgeführt worden. Lediglich 24\% der Ärzte lehnten indirekte Sterbehilfe ab. 15\% der Niedergelassenen sowie 11\% der Krankenhausärzte hatten bereits Nachfragen nach Beihilfe zum Suizid erhalten. Diese erfolgten aber in deutlich geringerem Maße als Bitten um aktive Sterbehilfe. Weiterhin konnten die Autoren aufzeigen, dass Krankenhaus- und niedergelassene Ärzte übereinstimmend sowohl den Begriff der Sterbehilfe $(77,8 \%$ der Krankenhausärzte und 85,3\% der Niedergelassenen) als auch den Begriff der Euthanasie (93,1\% der Krankenhausärzte und $84,3 \%$ der Niedergelassenen) für ungeeignet hielten. Schon bevor die Diskussion um Sterbehilfe in den deutschen Medien verstärkt thematisiert wurde, äußerten 50\% der Teilnehmer der im Herbst 1996 durchgeführten Studie einen gesteigerten Diskussionsbedarf. Die Untersuchung kann zwar als die erste bundesweit durchgeführte Studie zur Sterbehilfe angesehen werden, die sich jedoch - wie die folgenden Untersuchungen - nicht mit Einflüssen zur Einstellung gegenüber der Sterbehilfe befasst.

H. Csef aus der medizinischen Poliklinik in Würzburg und B. Heindl aus der Abteilung für allgemeine klinische und psychosomatische Medizin in Heidelberg veröffentlichten 1998 ihre Untersuchung zu Einstellungen deutscher Ärzte zur Sterbehilfe (Csef und Heindl 1998). Von den per Zufallsverfahren ausgewählten 93 Ärzten (44,1\% Klinikärzte; 45,2\% Niedergelassene) des ärztlichen Kreis- 
verbandes Würzburg (darunter 24 Allgemeinärzte) äußerten 34\% in dem durchgeführten halbstandardisierten Interview Zustimmung zur Durchführung passiver Sterbehilfe; 51\% unter bestimmten Bedingungen. Genannte Bedingungen waren hierbei vor allem die „Ausschöpfung aller anderen therapeutischen Maßnahmen“ sowie die „Aufklärung des Patienten über die potentiell lebensverkürzende Wirkung des Analgetikums“ und „eine Dosis, so hoch als nötig, aber so niedrig als möglich“ (Csef und Heindl 1998, S. 1503). 29\% der Ärzte hatten bereits Nachfragen nach aktiver Sterbehilfe erhalten. Die überwiegende Mehrheit (82\%) bezog gegen aktive Sterbehilfe Stellung; 9,7\% konnten sich hingegen aktive Sterbehilfe bei moribunden Patienten vorstellen. Alle Ärzte lehnten Sterbehilfe bei nicht moribunden Patienten ab. Fast die Hälfte der befragten Ärzte (44\%) äußerte die Ansicht, wonach es „Situationen gäbe, in denen menschliches Leben nicht lebenswert sei“ (Csef und Heindl 1998, S. 1503). Auch diese Studie ist in ihrer Aussagekraft durch die Vermengung verschiedener Fachrichtungen insofern eingeschränkt, als dass eine selektive Auswertung von Besonderheiten einzelner Fachdisziplinen erschwert wird. Weiterhin ist diese Studie durch die selektive Auswahl von Ärzten des Kreisverbandes Würzburg eingeschränkt.

Eine weitere Erhebung unter allen Ärzten, die an Fortbildungen des Tumorzentrums RheinlandPfalz teilgenommen hatten, wurde im Herbst 2001 von M. Weber et al. veröffentlicht (Weber et al. 2001). Von den 772 Teilnehmern waren 276 niedergelassene Ärzte. 86\% aller Befragten sprachen sich für die Möglichkeit der Beendigung lebensverlängernder Maßnahmen aus; $40 \%$ hatten bereits den Wunsch nach Sterbehilfe von Patientenseite erhalten. Die weit überwiegende Mehrheit (94\%) gab an, dass Sterbehilfe nur in Übereinstimmung mit dem Willen des Patienten durchgeführt werden dürfe. Als „sehr wichtig“ wurde die Ermittlung des „mutmaßlichen Willen(s) des Patienten“ durch eine Patientenverfügung von 79\% der Ärzte erachtet (Weber et al. 2001, A 3186). Nur 13\% der Ärzte hätten für sich selbst eine Patientenverfügung ausgestellt. Zwischen niedergelassenen und Krankenhausärzten ergaben sich in der Haltung zur Sterbehilfe keine relevanten Unterschiede. Die Autoren folgerten aber, dass bei den befragten Ärzten „große Meinungsunterschiede und Unterschiede bestehen, welche Maßnahmen denn tatsächlich beendet werden dürfen, und was zur unverzichtbaren Basisbetreuung gehört. Erhebliche Unsicherheiten bestehen auch hinsichtlich der juristischen Konsequenzen“ (Weber et al. 2001, A 3188). Diese Studie zeigt, dass sowohl in der Basisbetreuung wie auch in der Beendigung lebenserhaltender Maßnahmen und der Sterbehilfe erhebliche Unsicherheiten bestehen und weitere Diskussionen zum Thema geboten sind. Allerdings sind die Ergebnisse der Studie durch systematische Fehler eingeschränkt: Mit der ausschließlichen Heranziehung von Ärzten, die an Fortbildungen des Tumorzentrums Rheinland-Pfalz teilgenommen hatten, werden Defizite ohnehin schon überdurchschnittlich geschulter Ärzte geschildert. Insofern kann angenommen werden, dass Unsicherheiten bei der Gesamtheit deutscher Ärzte noch weitreichender sind. Zudem weisen die Autoren keine Ergebnisse vor, die Bezüge zur persönlichen Situation der teilnehmenden Ärzte erlauben und zur Beurteilung der Ergebnisse erforderlich sind. 
Diese Einschränkung konnte auch in der Auswertung der aktuellen Studie von Müller- Busch aus dem Jahre 2002 (Müller- Busch et al. 2003) festgestellt werden. In dieser Erhebung wurden Daten an einem selektionierten Kollektiv mit besonderen Erfahrungen in der Versorgung Schwerstkranker und sterbender Patienten erhoben. Hierzu wurden 730 Mitglieder bzw. 147 assoziierte Mitglieder der Deutschen Gesellschaft für Palliativmedizin per Fragebogenerhebung befragt. Eine deutliche Mehrheit von diesen lehnte die Legalisierung der aktiven Sterbehilfe (90\% bzw. 73\%) sowie die Beihilfe zum Suizid (74\% bzw. 59\%) ab. Die Hälfte (50\%) aller teilnehmenden Ärzte wünschte sich eine gesetzliche Regelung für eine Therapiebeendigung bei infausten Prognosen, in denen keine ausdrückliche Willensbekundung (Patientenverfügung, Vorsorgevollmacht) vorliegt. 3\% bzw. 1\% gaben an, bereits selbst aktive Sterbehilfe oder Beihilfe zum Suizid durchgeführt zu haben. Die historischen Erfahrungen mit Tötungen im Nationalsozialismus hatten in dieser Befragung keinen signifikanten Einfluss auf das Antwortverhalten der befragten Ärzte.

\section{Fragestellung und Ziel}

Die beschriebenen internationalen Studien und wenigen Untersuchungen aus Deutschland machen deutlich, dass ein großer Klärungsbedarf zum Thema "Sterbehilfe" besteht. Vom medizinischen Standpunkt aus soll diese Arbeit die momentan noch zurückhaltend geführte Diskussion zum Thema fortsetzen und Daten zur Realität von Behandlungsbegrenzung und -verzicht darstellen. Ebenso muss diskutiert werden, ob die aktuellen Regelungen zur Sterbehilfe der derzeitigen Versorgungsrealität entsprechen. Denn zumindest die passive Sterbehilfe wird - wie bereits gezeigt - von vielen Ärzten befürwortet. Diese Dissertation soll empirisch Einstellungen und Verhaltensweisen deutscher Hausärzte zur Sterbehilfe untersuchen, da in Deutschland bislang noch keine systematisch wissenschaftlich ausgewerteten Daten mit Rückschlussmöglichkeiten auf die Einflussfaktoren existieren (vgl. Wienke 2000). Insbesondere gibt es keine gesonderte Erhebung zur Haltung von Allgemein- und Hausärzten zur Sterbehilfe. Es sind ebenso keine Daten zur Häufigkeit von Nachfragen und Durchführung von aktiver und passiver Sterbehilfe im hausärztlichen/ familienmedizinischen Bereich vorhanden.

Die Beschäftigung mit dem Thema "Sterbehilfe" erweist sich rasch als komplexe Angelegenheit: der Zugang ist notwendigerweise interdisziplinär und schließt juristische und historische Überlegungen mit ein, die auch in der vorliegenden Arbeit berücksichtigt werden sollen.

\section{Material und Methoden}

\subsection{Die Ärzteerfassung}

Aus dem Gesamtkollektiv der niedergelassenen 32435 Allgemeinärzte in der Bundesrepublik Deutschland wurden über eine CD-ROM alle bis zum April 2001 verzeichneten Ärzte erfasst. Aus 
diesem Kollektiv wurden per EDV-automatisiertes Zufallsverfahren (Randomisation) 500 Ärzte ausgewählt und um Teilnahme an den Untersuchungen gebeten.

\subsection{Der Erhebungsbogen}

Die Erhebung erfolgte über einen Fragebogen, der im Rahmen der vorliegenden Arbeit entwickelt wurde. Neben der Erhebung über die Haltung deutscher Allgemeinärzte zur Sterbehilfe sollte auch die Möglichkeit des Vergleichs mit Ärzten aus anderen Nationen gegeben sein. Daher wurde sich neben den neu erarbeiteten Fragen und Passagen auch an Fragen bereits durchgeführter Erhebungen in anderen Ländern orientiert. Insbesondere die Fallvignetten wurden aus Gründen der Vergleichbarkeit nach Übersetzung in Fragestellung und Antwortmöglichkeiten wortgleich übernommen (Emanuel et al. 1996). Da im deutschen Sprachraum überwiegend die Unterscheidung von aktiver und passiver Sterbehilfe gebräuchlich ist, fanden nur diese Begriffe im Fragebogen Verwendung. Der im Angelsächsischen verwendete Begriff des „physician-assisted suicide“- der Beihilfe zur Selbsttötung - blieb daher unerwähnt.

In den meisten internationalen Arbeiten zur aktiven und passiven Sterbehilfe, zur Euthanasie oder der ärztlichen Beihilfe zum Suizid fehlen einheitliche Definitionen. Auch in den wenigen deutschen Untersuchungen zum Thema wurde festgestellt, dass viele deutsche Ärzte Probleme mit der Abgrenzung der Begriffe aktive und passive Sterbehilfe haben (Kirschner und Elkeles 1998, MüllerBusch et al. 2003). Es war daher notwendig, als Ausgangsposition eine Beschreibung von Sachverhalten und Definitionen zu finden, die eine allgemeine Akzeptanz vermuten ließen. In Zusammenarbeit mit der Abteilung für Ethik und Geschichte der Medizin der Universität Göttingen wurden in Anlehnung an internationale Publikationen (Council of the Royal College of Physicians 2001) die folgenden Definitionen zur aktiven und passiven Sterbehilfe entwickelt. Diese waren dem Fragebogen zur begrifflichen Klärung vorangestellt.

Angesichts der problematischen Definition der Sterbehilfe wurden die teilnehmenden Ärzte gebeten, den Fragebogen unter Zugrundelegung der gegebenen Definitionen zu beantworten.

Aktive und passive Sterbehilfe wurde wie folgt definiert:

Aktive Sterbehilfe:

„Als aktive Sterbehilfe gelten diejenigen ärztlichen Maßnahmen, bei denen durch aktives Handeln und Eingreifen des Arztes der Tod des Patienten ausgelöst wird, nachdem dieser den Arzt darum gebeten hat. Beispiele hierfür sind die „aktive“ Injektion oder Verabreichung eines todbringenden Medikamentes durch den Arzt."

Passive Sterbehilfe:

„Als passive Sterbehilfe gelten diejenigen ärztlichen Maßnahmen, bei denen einem sterbewilligen Patienten die Mittel zur Herbeiführung seines Todes an die Hand gegeben werden, ohne dass der 
Arzt selbst handelnd tätig wird. Beispiele hierfür sind die Überlassung starker Analgetika, bei deren Einnahme die Möglichkeit besteht, dass der Patient durch Nebenwirkungen (z.B. Atemdepression) verstirbt."

Der eigentliche Fragebogen bestand aus vier Teilen.

Im ersten Teil des Erhebungsbogens wurden die Ärzte gebeten, zu insgesamt vier hypothetischen Fallvignetten Stellung zu nehmen. Die Angaben sollten unter der Annahme erfolgen, dass sowohl aktive als auch passive Sterbehilfe legal seien. Diese Fallvignetten orientierten sich an internationalen Untersuchungen und fragen in abgestufter Form Entscheidungen zur Sterbehilfe ab (Emanuel et al. 1996). Allen Fallvignetten gemeinsam war das Thema eines zurechnungsfähigen, nicht depressiven Patienten, der von seinem Arzt bereits lange betreut wird und inm gut bekannt ist. Er leidet an einem fortgeschrittenen Malignom und hat nur noch wenige Monate zu leben. Über diese Konstanten hinaus variierten die Fallvignetten voneinander in folgenden Punkten:

In der ersten Fallvignette leidet der Patient unter nicht therapierbaren Schmerzen durch Metastasen und bittet deshalb den betreuenden Arzt um Sterbehilfe.

Im zweiten Fall steht die Sorge um die Belastung der Familie durch die fortschreitende Erkrankung im Vordergrund.

In der dritten Vignette bittet der Patient um Sterbehilfe, da zunehmende Schwierigkeiten bei der eigenen Versorgung befürchtet werden.

Im letzten Fallbeispiel sieht der Patient keinen Sinn darin, weiter zu leben und bittet deshalb um die Durchführung von Sterbehilfe.

Zu diesen Fallvignetten konnten die Teilnehmer aus fünf Antwortmöglichkeiten eine wählen, die sich aus Gründen der Vergleichbarkeit ebenfalls an den Antwortmöglichkeiten internationaler Untersuchungen orientierten:

1. Durchführung palliativer Maßnahmen; Ablehnung von Sterbehilfe

2. Verordnung von Morphium und Instruktion der letalen Einnahme

3. Verzicht auf weitere Behandlung

4. Verabreichung einer tödlichen Medikamenteninjektion

5. Darüber hinaus war die Möglichkeit gegeben, in Form von Freitext Vorschläge zu äußern, falls keine der vorangegangenen Antwortmöglichkeiten auf Zustimmung stoßen sollte

Im zweiten Teil des Fragebogens wurden insgesamt 13 allgemeine Fragen zum Thema Sterben und Sterbehilfe gestellt. Hier sollten Häufigkeiten der Nachfrage nach Sterbehilfe sowie persönliche Einschätzungen und Beweggründe erfragt werden. Ein weiterer Teil beinhaltete Fragen zu 
Wünschen nach gesetzlichen Regelungen in Deutschland und Fragen zur Beurteilung internationaler Modelle zur Sterbehilfe. Hierbei gab es sowohl Einfach- als auch Mehrfachantwortmöglichkeiten mit der zusätzlichen Option eines eigenen Kommentars.

Im letzten Teil wurden soziodemographische Angaben der Teilnehmer erfragt. Insbesondere fanden Alter und Geschlecht, Familienstand, Konfessionszugehörigkeit sowie Tätigkeitsbereiche und Angaben zur eigenen Praxis Berücksichtigung. Auch hier wurde am Ende des Bogens noch Platz für eigene Anmerkungen gelassen. Eine Ausfertigung des Fragebogens ist im Anhang beigefügt.

\subsection{Pilotstudie und Versenden der Fragebögen}

Die Pilotierung des Fragebogen-Entwurfes erfolgte im Forschungskurs der Deutschen Gesellschaft für Allgemeinmedizin und Familienmedizin in Freiburg im Mai 2001. Es wurden hierbei an 25 Allgemeinärzte Fragebögen verteilt, von denen 17 zurückgesandt wurden. Diese gingen in die Vorauswertung ein. Nach erneuter Überarbeitung des Bogens wurde die endgültige Fassung des Bogens erstellt. Dieser wurde im Juli 2001 an die im zuvor genannten Zufallsverfahren ausgewählten Ärzte versandt. Den Fragebögen wurden je ein Anschreiben sowie Hinweise zur Beantwortung des Fragebogens mit den genannten Definitionen zur Sterbehilfe beigelegt. In dem Anschreiben wurden die potentiellen Teilnehmer über Hintergrund und Durchführung der Untersuchung informiert. Außerdem wurde ihnen ausdrücklich die vollständige Anonymisierung ihrer Daten zugesichert. Die Rücksendung konnte mittels eines frankierten Rückumschlags erfolgen. Sollten Teilnehmer Interesse an den Ergebnissen der Untersuchungen bekunden, so wurde aus Anonymisierungsgründen zusätzlich ein separater Faxvordruck hinzugefügt. Die Faxantwort gab dem angeschriebenen Arzt zudem die Möglichkeit um einen Rückruf zu bitten, falls es Unklarheiten bei der Beantwortung der Fragen gab oder noch zusätzliche Informationen zum Thema gewünscht wurden.

Um die Antwortrate zu erhöhen, wurde einen Monat später ein Erinnerungsschreiben an alle Ärzte verschickt, das ebenfalls aus Anschreiben, Fragebogen, Rückumschlag und Faxantwort bestand. Zwei Monate nach diesem Erinnerungsschreiben und insgesamt drei Monate nach dem ersten Anschreiben wurde mit den Auswertungen begonnen.

Drei Fragebögen, die nach dieser Zeit eintrafen, wurden nicht mehr in die Auswertung miteinbezogen.

\subsection{Bearbeitung der Erhebungsbögen}

Die Erfassung und die statistischen Analysen der zurückgesandten Fragebögen erfolgte EDVgestützt (SAS 8.1; SAS Institute Cary, NC 27513, USA). 
Bei einigen Antwortmöglichkeiten bestand zusätzlich die Möglichkeit des freien Kommentars. Dieser wurde separat ausgewertet. Eine Mehrfachangabe bei vorgesehener Einfachauswahl wurde als ungültig gewertet.

\subsection{Statistische Verfahren}

Die Analyse des statistischen Zusammenhangs von Merkmalen der untersuchten Ärztegruppe wurde mit Hilfe des Chi-Quadrat-Tests durchgenommen. Es wurde zweiseitig mit einer Irrtumswahrscheinlichkeit von $\alpha=0,05$ getestet.

Faktoren, die alleine getestet einen signifikanten Einfluss auf die Akzeptanz aktiver bzw. passiver Sterbehilfe zeigten, sowie Faktoren, deren Kontrolle für die Beantwortung der Frage wichtig erschienen, wurden in einer logistischen Regression erneut auf ihre Bedeutung hin überprüft. Abhängige Variable war in beiden Modellen die Antwort auf die Frage, inwiefern die Ärzte aktive bzw. passive Sterbehilfe für ein akzeptables Vorgehen halten (Frage 5). Als abhängige Variablen wurde folgende ausgewählt: Das (Mit)erleben einer lebensbedrohlichen Krankheit (Frage 1), erhaltene Nachfragen nach aktiver bzw. passiver Sterbehilfe (Frage 2), das Alter (kategorisiert in Quartile), Geschlecht, Familienstand, eigene Kinder, die Konfession und ob die Ärzte bereits medizinische Erfahrungen in den Bereichen Onkologie oder Geriatrie gesammelt hatten. Es wurde eine Rückwärtselektion mit einem Signifikanzlevel für die Variablenselektion von $\alpha=0,05 \mathrm{im}$ Likelihood ratio test gewählt. Die Ergebnisse werden in Odds ratios mit 95\% Konfidenzintervallen dargestellt.

\subsection{Limitierung der Studie}

Die vorliegende Arbeit versucht, repräsentativ Haltungen und Einstellungen von Allgemeinärzten der gesamten Bundesrepublik zur Sterbehilfe darzustellen.

Allerdings ist die Auswahl der teilnehmenden Ärzte dadurch eingeschränkt, dass aufgrund datenschutzrechtlicher Bedenken die Kassenärztlichen Vereinigungen keine Liste deutscher Allgemeinärzte zur Verfügung stellen konnten. Als Ersatzverfahren erfolgte die randomisierte Auswahl der Ärzte über ein telephonisches Gesamtverzeichnis (Deutsche Telekom) aller deutschen Allgemeinärzte. Es muss daher berücksichtigt werden, dass mit dieser Untersuchung diejenigen Ärzte nicht erreicht werden konnten, die über keinen Telefonanschluß in ihrer Praxis verfügen. Angesichts heutiger Kommunikationsstrukturen darf jedoch mit einer hinreichenden Sicherheit angenommen werden, dass allenfalls in Ausnahmefällen Ärzte in Deutschland nicht im Telefonverzeichnis vermerkt sind. Ebenfalls keine Berücksichtigung findet der sicher zahlenmäßig zu vernachlässigende Anteil rein privatärztlich tätiger Allgemeinärzte. 
Ausgehend von einer Gesamtzahl von 32435 Allgemeinärzten in Deutschland und einem gewünschten Recall von 50\% wurde eine Gesamtzahl von 500 Ärzten einkalkuliert, um etwa 1\% aller deutschen Allgemeinärzte zu erreichen.

\section{Ergebnisse}

Von insgesamt 481 Fragebögen sind 233 auswertbare zurückgesendet worden. Das entsprach einer Antwortrate von 48,44\%.61,80\% der Faxantworten wurden mit der Bitte um Zusendung der Ergebnisse der Studie wieder zurückgesendet. 19 Fragebögen kamen ungelesen wieder zurück. Die erkennbaren Gründe hierfür waren vielschichtig: einige Adressaten waren unbekannt verzogen $(n=5)$, innerhalb der letzten Monate pensioniert $(n=5)$, hatten die Fachrichtung geändert $(n=3)$ oder waren erkrankt $(n=6)$.

Das durchschnittliche Alter der teilnehmenden Ärzte betrug 51 Jahre (Spannweite 29-77 Jahre), $67,67 \%$ waren männlich, $79,82 \%$ verheiratet und $84,05 \%$ hatten Kinder. Jeder Dritte $(32,67 \%)$ war katholisch, 38,36\% evangelisch und ein Viertel (25,43\%) hatte keine Konfession. Drei Viertel $(74,67 \%)$ der Ärzte arbeiteten in einer Einzelpraxis. Eine Mehrheit $(65,95 \%)$ gab an sie selbst oder Verwandte hätten bereits an einer lebensbedrohlichen Krankheit gelitten.

Einen Überblick über soziodemographische Daten und weitere für die Befragung relevante Charakteristika der teilnehmenden Ärzte gibt Tabelle 3.

\begin{tabular}{|c|c|c|}
\hline Stichprobe (Antwortrate) & $n=233 / 481(48,44 \%)$ & \\
\hline Alter & Durchschnitt: 51 Jahre (Spannweite & 29-77), s= 3 Jahre \\
\hline Geschlecht & $\begin{array}{l}\text { männlich } \\
\text { weiblich }\end{array}$ & $\begin{array}{l}n=157(67,67 \%) \\
n=75(32,33 \%)\end{array}$ \\
\hline Familienstand & $\begin{array}{l}\text { verheiratet / eheähnl. Gemeinschaft } \\
\text { ledig } \\
\text { geschieden/ getrennt lebend } \\
\text { verwitwet }\end{array}$ & 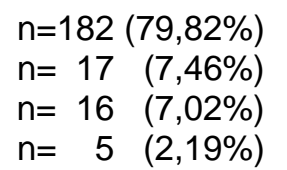 \\
\hline Kinder & $\begin{array}{l}\text { mit Kindern } \\
\text { ohne Kinder }\end{array}$ & $\begin{array}{l}n=195(84,05 \%) \\
n=37(15,95 \%)\end{array}$ \\
\hline Religion & $\begin{array}{l}\text { katholisch } \\
\text { evangelisch } \\
\text { andere } \\
\text { keine }\end{array}$ & $\begin{array}{l}\mathrm{n}=76(32,76 \%) \\
\mathrm{n}=89(38,36 \%) \\
\mathrm{n}=8(3,45 \%) \\
\mathrm{n}=59(25,43 \%)\end{array}$ \\
\hline Religionsausübung & $\begin{array}{l}\text { aktiv } \\
\text { gelegentlich aktiv } \\
\text { nicht aktiv }\end{array}$ & $\begin{array}{l}n=38(19,69 \%) \\
n=64(33,16 \%) \\
n=91(47,15 \%)\end{array}$ \\
\hline
\end{tabular}




\begin{tabular}{|c|c|c|}
\hline Medizinische Tätigkeitsbereiche/Erfahrungen & $\begin{array}{l}\text { Innere Medizin } \\
\text { Geriatrie } \\
\text { Onkologie } \\
\text { Psychiatrie } \\
\text { andere }\end{array}$ & $\begin{array}{l}n=168(74,34 \%) \\
n=94(41,59 \%) \\
n=70(30,84 \%) \\
n=39(17,26 \%) \\
n=124(54,87 \%)\end{array}$ \\
\hline Jahr der Approbation & $\begin{array}{l}<18 \text { Jahre zurückliegend } \\
18-36 \text { Jahre zurückliegend } \\
>36 \text { Jahre zurückliegend }\end{array}$ & $\begin{array}{l}\mathrm{n}=63(27,88 \%) \\
\mathrm{n}=139(61,50 \%) \\
\mathrm{n}=24(10,62 \%)\end{array}$ \\
\hline Tätigkeit in der eigenen Praxis & \multicolumn{2}{|c|}{ Durchschnitt: 15 Jahre (Spannweite 0,5-40), s= 1 Jahr } \\
\hline Art der Praxis & $\begin{array}{l}\text { Einzelpraxis } \\
\text { Gemeinschaftspraxis } \\
\text { Praxisgemeinschaft }\end{array}$ & $\begin{array}{l}n=171(74,67 \%) \\
n=43(18,78 \%) \\
n=15(6,55 \%)\end{array}$ \\
\hline Ort der Praxis (Einwohner) & $\begin{array}{l}<10.000 \\
11-50.000 \\
51-100.000 \\
>100.000\end{array}$ & $\begin{array}{l}n=85(36,64 \%) \\
n=89(38,38 \%) \\
n=18 \quad(7,76 \%) \\
n=40 \quad(17,24 \%)\end{array}$ \\
\hline Praxisgröße & \multicolumn{2}{|c|}{$\begin{array}{l}\text { Durchschnitt: } 1.137 \text { Patienten/ Quartal } \\
\text { (Spannweite 100- 3.000), s= } 76 \text { Patienten/ Quartal }\end{array}$} \\
\hline
\end{tabular}

\subsection{Deskriptive Auswertungen der Fallvignetten}

Den Antworten bei allen vier Fallvignetten war gemeinsam, dass die Möglichkeit „Ich verzichte auf die weitere Behandlung des Patienten“ nie angekreuzt worden ist. Die Durchführung palliativer Maßnahmen dagegen war in jedem Fallbeispiel die favorisierte Antwortmöglichkeit. Häufig wurde bei der Beantwortung einer der Fallvignetten die Möglichkeit eines "anderen", d.h. freien Antworttextes genutzt (1. Fallvignette: 9,42\%; 2. Fallvignette: 10,30\%; 3. Fallvignette: 6,44\%; 4. Fallvignette: 5,56\%). Diese Kommentare beinhalteten in jedem der vier Fallbeispiele primär die Durchführung noch intensiverer palliativer Maßnahmen als die bereits im Beispiel vorgegebenen, sinnfindende Gespräche mit dem Patienten, Einbindung der Familie und des Hospizes sowie die Klärung letzter offener persönlicher Angelegenheiten.

\section{Fallvignette}

" Ein voll zurechnungsfähiger Patient leidet an einem fortgeschrittenem Prostata-Karzinom mit Knochenmetastasen und hat voraussichtlich nur noch wenige Monate zu leben. Es besteht keine Aussicht auf eine Heilung oder eine Verminderung des Krankheitsprogresses. Trotz Ausschöpfung aller palliativer Maßnahmen wie NSAID, Morphin, Strahlentherapie, Nervenblockaden etc. verursachen die Metastasen unerträgliche Schmerzen. Sie betreuen den Patienten seit zwei Jahren. Er wurde von einem Psychiater als nicht depressiv beurteilt und bittet Sie nun zum wiederholten Male um Sterbehilfe." 
Im ersten Fallbeispiel gab über die Hälfte der befragten Ärzte an, palliative Maßnahmen durchführen zu wollen. Etwa ein Drittel entschied sich für die Morphinverabreichung und jeder zehnte würde keine der angegebenen Maßnahmen durchführen. Knapp drei Prozent würden den betroffenen Patienten eine tödliche Dosis eines Medikamentes spritzen (Abbildung 1).

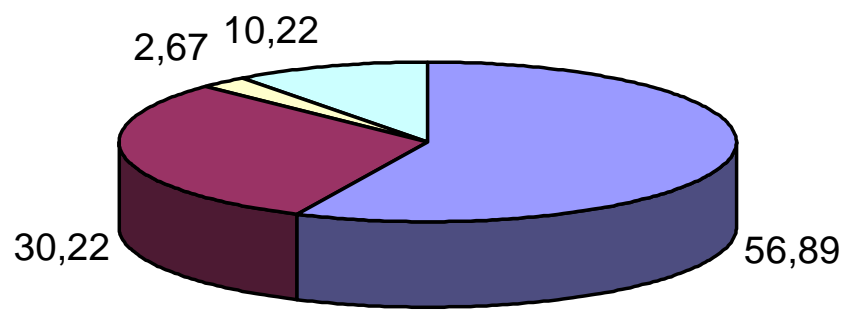

\begin{tabular}{|l|}
\hline Palliation \\
$\square$ aktive Sterbehilfe \\
$\square$ passive Sterbehilfe \\
$\square$ andere Möglichkeit
\end{tabular}

\% der befragten Ärzte

Abbildung 1: Handlungsweisen in der 1. Fallvignette $(n=225)$

2. Fallvignette

" Ein voll zurechnungsfähiger Patient leidet unter einem fortgeschrittenen Malignom und hat voraussichtlich nur noch wenige Monate zu leben. Es bestehen keine Aussichten auf eine Heilung oder eine Verminderung des Krankheitsprogresses. Unter der jetzigen Therapie ist der Patient schmerzfrei und kann für sich selbst sorgen. Er ist jedoch zunehmend besorgt über die Belastung, die durch die Verschlechterung der Krankheit und den Tod auf seine Familie zukommen wird. Der Patient wurde von einem Psychiater als klinisch nicht depressiv beurteilt, fragt aber zum wiederholten Male nach einer lebensbeendenden Injektion. "

Bei der zweiten Fallvignette gab wiederum die Mehrheit an, palliativen Maßnahmen den Vorrang einzuräumen. Jedoch entschieden sich die Ärzte bei diesem Fallbeispiel als zweithäufigste Antwort für eine „andere“ als die angegebenen Möglichkeiten. Danach folgte die Morphinverordnung. Ein einziger Arzt gab an, dem Patienten eine tödliche Menge eines Medikamentes zu verabreichen (Abbildung 2). 

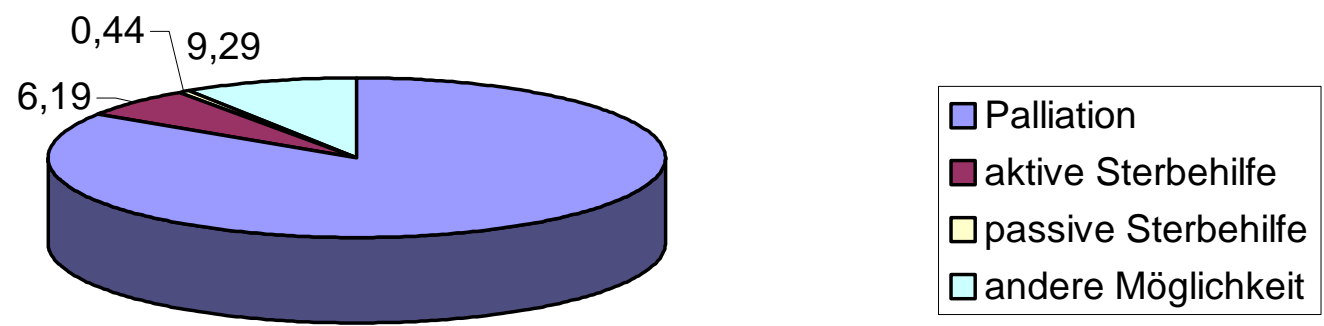

84,07

$\%$ der befragten Ärzte

Abbildung 2: Handlungsweisen in der 2. Fallvignette $(n=226)$

\section{Fallvignette}

" Ein voll zurechnungsfähiger Patient leidet unter einem fortgeschrittenen Malignom und hat voraussichtlich nur noch wenige Monate zu leben. Es bestehen keine Aussichten auf eine Heilung oder eine Verminderung des Krankheitsprogresses. Unter der jetzigen Therapie ist der Patient schmerzfrei. Er kann jedoch weder das Bett verlassen noch sich selbst versorgen. Der Patient wurde von einem Psychiater als klinisch nicht depressiv beurteilt, fragt aber zum wiederholten Male nach einer lebensbeendenden Injektion. "

Bei dem dritten Fallbeispiel wählten erneut über drei Viertel der Befragten die erste Antwortmöglichkeit aus, die die Ausschöpfung palliativer Maßnahmen beinhaltete. Darauf folgte die Morphinverordnung und ein kleiner Teil der Teilnehmer würde keine der angegebenen Möglichkeiten durchführen. Eine Minderheit (1,76\%) würde den betroffenen Patienten eine letale Medikamentendosis injizieren (Abbildung 3).
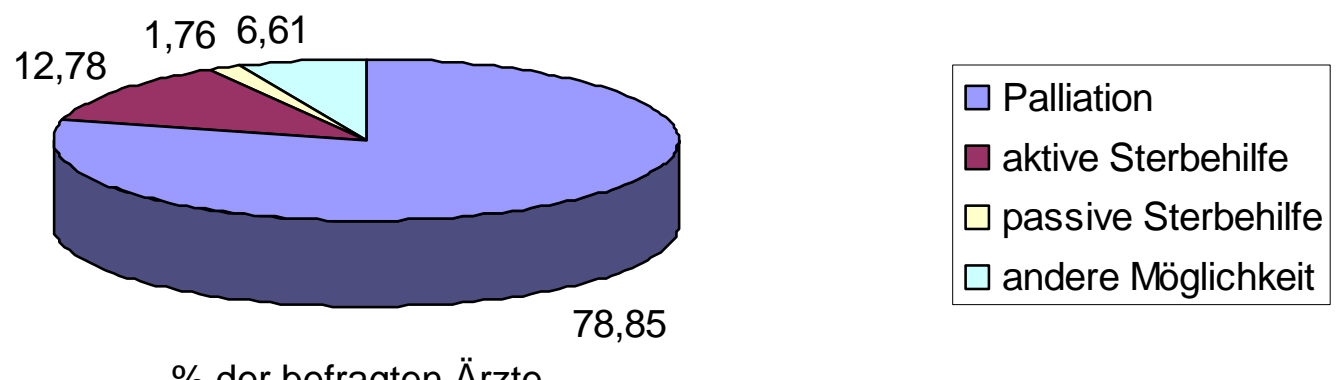

$\%$ der befragten Ärzte

Abbildung 3: Handlungsweisen in der 3. Fallvignette $(n=227)$

\section{Fallvignette}

" Ein voll zurechnungsfähiger Patient leidet unter einem fortgeschrittenen Malignom und hat voraussichtlich nur noch wenige Monate zu leben. Es bestehen keine Aussichten auf eine Heilung oder eine Verminderung des Krankheitsprogresses. Unter der jetzigen Therapie ist der Patient 
schmerzfrei und kann sich selbst versorgen, findet sein Leben aber sinn- und zwecklos. Der Patient wurde von einem Psychiater als klinisch nicht depressiv beurteilt, fragt aber zum wiederholten Male nach einer lebensbeendenden Injektion. "

Die letzte Fallvignette ähnelte in ihrer Antwortkonstellation der zweiten. Während sich wiederum eine breite Mehrheit für die Ablehnung von Sterbehilfe und für die Durchführung palliativer Maßnahmen aussprach, würde nur ein kleiner Teil den betroffenen Patienten Morphium verordnen. Etwa jeder Zehnte entschied sich für eine „andere“ Antwortmöglichkeit. Auch bei diesem Beispiel gab ein Arzt an, gegebenenfalls eine tödliche Menge eines Medikamentes zu verabreichen (Abbildung 4).

$$
0,44
$$

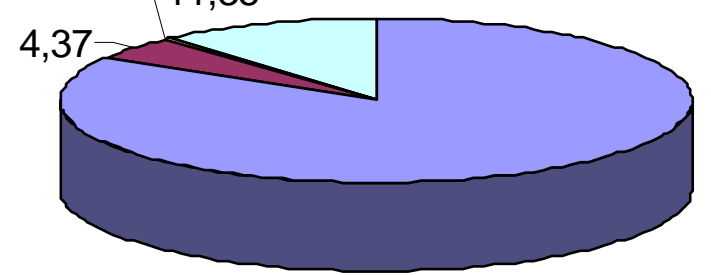

83,84

$\%$ der befragten Ärzte

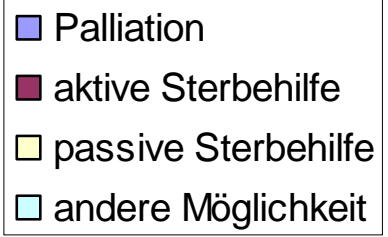

$\square$ andere Möglichkeit

Abbildung 4: Handlungsweisen in der 4. Fallvignette $(n=229)$

\subsection{Allgemeine Fragen}

Von den teilnehmenden Ärzten hatten bereits 65,95\% selbst oder ein Angehöriger an einer lebensbedrohlichen Krankheit gelitten.

Von Patienten um Sterbehilfe gebeten, würden die teilnehmenden Ärzte wie folgt reagieren (Abbildung 5): 


\begin{tabular}{|l}
$\square$ Beratung mit \\
anderen \\
$\square$ Psychiatrische Untersuchung \\
des Patienten \\
$\square$ Aufzeigen von Alternativen \\
für den Patienten \\
$\square$ Durchführung von \\
passiver Sterbehilfe \\
$\square$ Durchführung von \\
aktiver Sterbehilfe \\
$\square$ Ablehnung von Sterbehilfe \\
\end{tabular}

$\square$ Beratung mit

Psychiatrische Untersuchung des Patienten

Durchführung von

aktiver Sterbehilfe

Ablehnung von Sterbehilfe

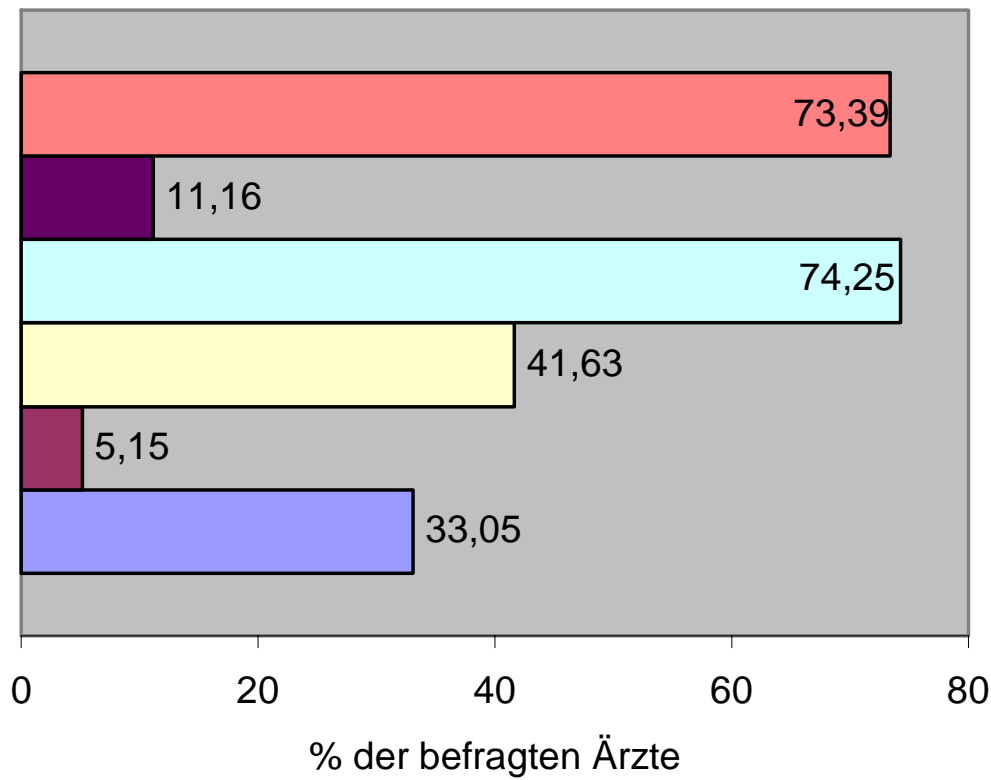

\% der befragten Ärzte

Abbildung 5: Reaktionen auf ein Sterbehilfegesuch ( $n=233$; Mehrfachantworten möglich)

Für die Hälfte der befragten Ärzte $(50,68 \%)$ bestand ein moralisch relevanter Unterschied zwischen der Durchführung aktiver und passiver Sterbehilfe; 7,24\% markierten bei dieser Frage die Antwortmöglichkeit "unsicher". Es wurde weiterhin gefragt, ob die Teilnehmer glaubten das Richtige getan zu haben, falls sie bereits Sterbehilfe geleistet hätten. Die Mehrheit beantwortete die Frage, ob sie bereits aktive $(86,70 \%)$ oder passive $(61,80 \%)$ Sterbehilfe geleistet hätten, nicht. Somit wurde diese Frage von 13,30\% für die aktive und entsprechend 38,20\% für die passive Sterbehilfe beantwortet. Daraus wurde geschlossen, dass diese Ärzte bereits Sterbehilfe durchgeführt hatten. Etwa jeder Zehnte $(12,50 \%$ von $n=31)$ bzw. nahezu jeder $(96,63 \%$ von $n=89)$ von diesen war davon überzeugt, das Richtige (aktive bzw. passive Sterbehilfe) getan zu haben.

Der Großteil der Befragten (90,56\%) gab an, noch nie Mittel zur Durchführung eines Suizids zur Verfügung gestellt zu haben. Auch bei dieser Frage war ein geringer Prozentsatz $(3,86 \%)$ "unsicher" bei der Beantwortung.

Nach den Favoriten internationaler Modelle zur Sterbehilfe befragt, entschied sich die Hälfte (51,00\%) für das deutsche Modell und je ein Viertel (24,5\%) für das schweizerische und niederländische.

Ferner gaben fast alle Ärzte $(88,65 \%)$ an, sich während ihrer Ausbildung zum Arzt nicht ausreichend mit den Themen Sterben und Sterbehilfe konfrontiert gesehen zu haben.

Weiterhin hielt eine Mehrheit (79,13\%) einen Vergleich zwischen der Euthanasie im sog. „Dritten Reich“ und der heutigen Durchführung von Sterbehilfe für nicht gerechtfertigt. 


\subsection{Fragen zur aktiven Sterbehilfe}

Weit mehr als die Hälfte (62,01\%) der befragten Ärzte gab an, bereits Anfragen nach aktiver Sterbehilfe erhalten zu haben. Einen Überblick über die Anzahl der erhaltenen Nachfragen innerhalb der letzten zehn Jahre gibt die folgende Abbildung:

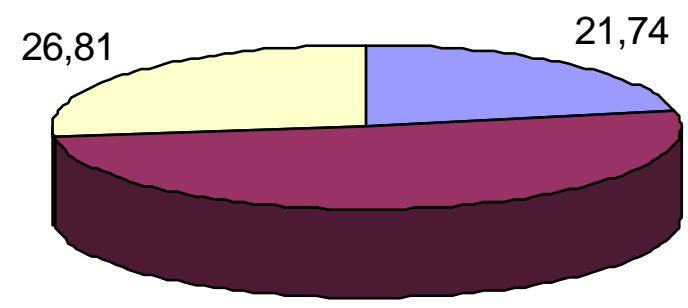

51,45

$\%$ der befragten Ärzte

Abbildung 6: Erhaltene Nachfragen nach aktiver Sterbehilfe in den letzten zehn Jahren $(n=229)$

Über ein Drittel $(34,72 \%)$ der befragten Ärzte hielt aktive Sterbehilfe unter bestimmten Umständen für ein akzeptables Vorgehen. Die angegebenen Beweggründe für die Durchführung aktiver Sterbehilfe sind in Abbildung 7 dargestellt:

\begin{tabular}{l}
\hline Entlastung des \\
Gesundheitssystems \\
$\square$ Befreiung der \\
Angehörigen von Lasten \\
$\square$ Ermöglichung eines \\
Sterbens in Würde \\
$\square$ Recht auf einen \\
selbstbestimmten Tod \\
$\square$ Erlösung des Patienten von \\
Schmerzen
\end{tabular}

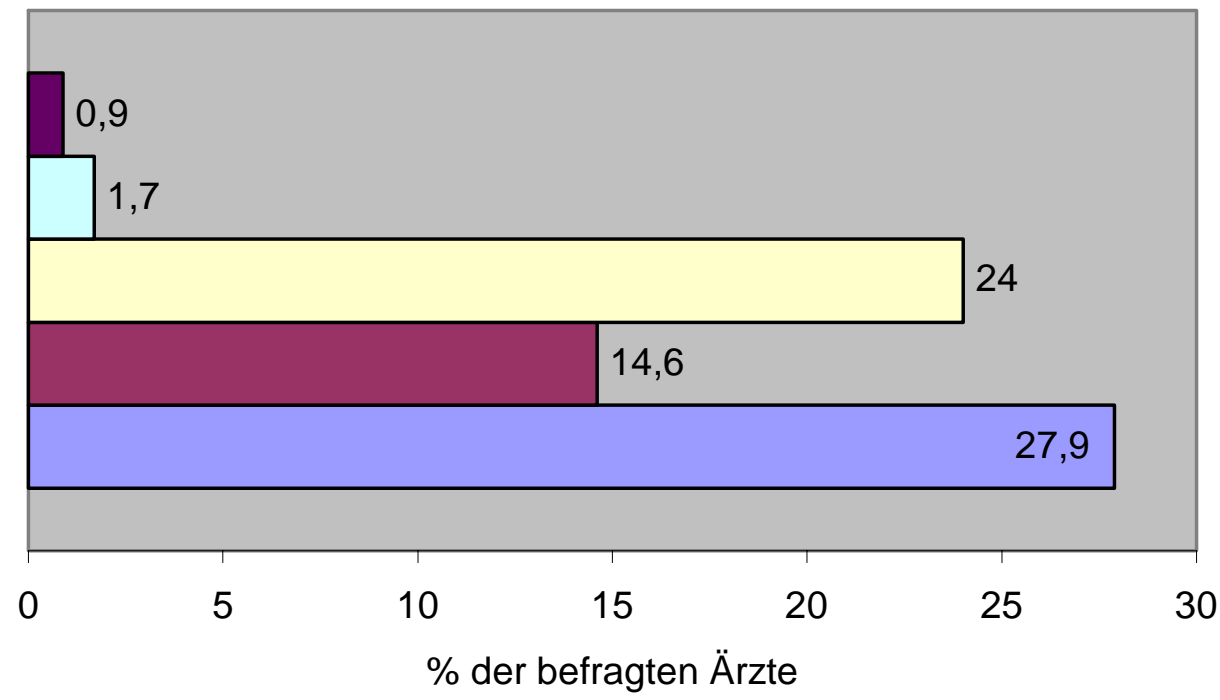

Abbildung 7: Beweggründe für die Durchführung von aktiver Sterbehilfe $(n=233$; Mehrfachnennungen möglich)

Beweggründe, Bitten um aktive Sterbehilfe zurückzuweisen, sind in Abbildung 8 aufgeführt: 


\begin{tabular}{|l|}
\hline Religiöse \\
Gründe \\
$\square$ Eid des \\
Hippokrates \\
$\square$ Historische Gründe \\
"Drittes Reich" \\
$\square$ Juristische \\
Gründe \\
$\square$ Ausreichende \\
Palliativmedizin \\
$\square$ Möglichkeit ärztlicher \\
Fehleinschätzung \\
$\square$ Möglichkeit des \\
Missbrauchs \\
\hline
\end{tabular}

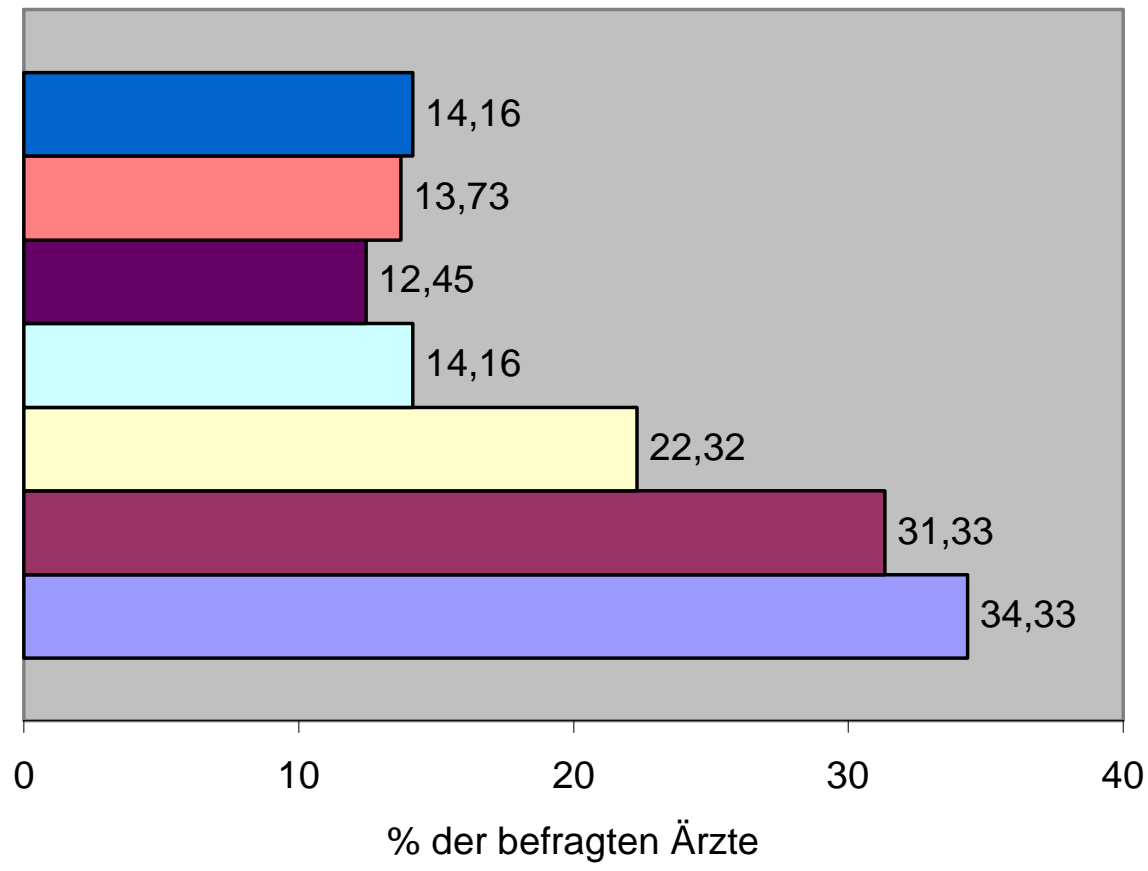

40

Abbildung 8: Beweggründe gegen die Durchführung von aktiver Sterbehilfe $(n=233$; Mehrfachnennungen möglich)

Nach den Gründen befragt, die die Entscheidung, aktive Sterbehilfe durchzuführen erleichtern könnten, wurden die in Abbildung 9 gezeigten Antworten gegeben: 
Keine strafrechtliche

Verfolgung

$\square$ Unabhängige Beurteilung durch andere Ärzte

$\square$ Wiederholter Wunsch des

Patienten

$\square$ Schriftlicher Wunsch

des Patienten

Zustimmung der

Angehörigen

$\square$ Ablehnung palliativer

Maßnahmen durch den Patienten

$\square$ Ausschluss einer psychischen

Erkrankung

$\square$ Volle

Zurechnungsfähigkeit

$\square$ Begrenzte

Lebenserwartung

$\square$ Nicht beherrschbare

Schmerzen

$\square$ Gute Kenntnis/Lange Betreuung des Patienten

$\square$ Mindestalter des

Patienten

$\square$ Andere Gründe

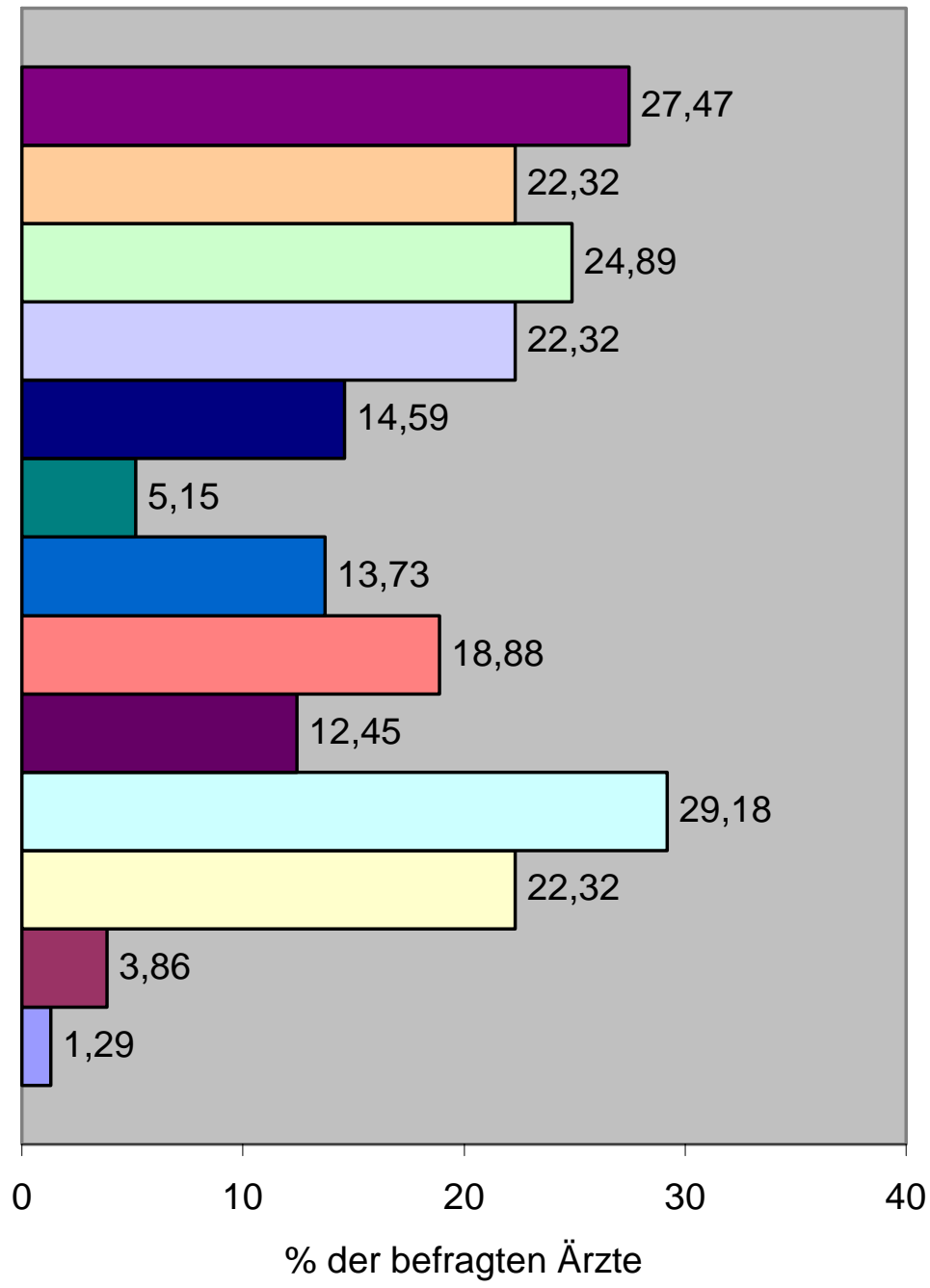

\% der befragten Ärzte

Abbildung 9: Gründe, die die Durchführung aktiver Sterbehilfe erleichtern würden $(n=233$; Mehrfachnennungen möglich)

Es konnte weiterhin festgestellt werden, dass die Hälfte der befragten Ärzte (49,53\%) eine gesetzliche Regelung der aktiven Sterbehilfe für erforderlich hielt. Vier Prozent (4,25\%) waren sich über diesen Punkt "unsicher". Knapp ein Fünftel (18,27\%) wäre bereit, aktive Sterbehilfe durchzuführen, wenn hierfür in Deutschland eine gesetzliche Grundlage bestünde. Ein geringerer Teil $(13,94 \%)$ der Ärzte war sich nicht sicher, ob er in diesem Fall aktive Sterbehilfe durchführen würde.

\subsection{Fragen zur passiven Sterbehilfe}

Etwa drei Viertel $(73,01 \%)$ der befragten Ärzte gab an, bereits um passive Sterbehilfe gebeten worden zu sein. Einen Überblick über die Anzahl der Nachfragen, die die Teilnehmer innerhalb der letzten zehn Jahren erhalten hatten, gibt die nachfolgende Abbildung (Abbildung 10): 


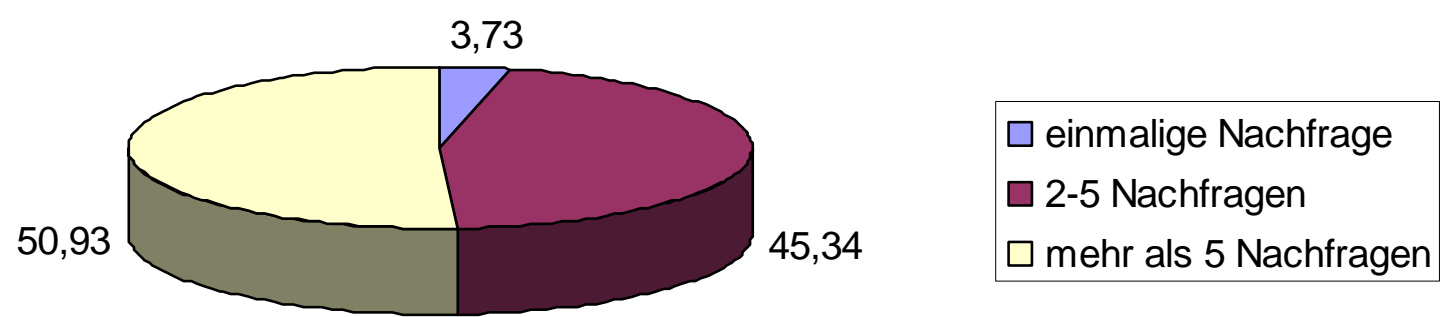

\% der befragten Ärzte

Abbildung 10: Nachfragen nach passiver Sterbehilfe in den letzten zehn Jahren

Mehr als drei Viertel (80,35\%) akzeptierte die Durchführung passiver Sterbehilfe. In Abbildung 11 sind Beweggründe für die individuelle Haltung zur Unterstützung passiver Sterbehilfe dargestellt:

\begin{tabular}{|l|}
\hline Religiöse \\
Gründe \\
$\square$ Entlastung des \\
Gesundheitssystems \\
$\square$ Befreiung der Angehörigen \\
von Lasten \\
$\square$ Ermöglichung eines \\
Sterbens in Würde \\
$\square$ Recht auf einen \\
selbstbestimmten Tod \\
$\square$ Erlösung des Patienten \\
von Schmerzen \\
\hline
\end{tabular}

Abbildung 11: $\quad$ Bewe

Beweggründe gegen die Durchführung passiver Sterbehilfe sind in Abbildung 12 aufgeführt:

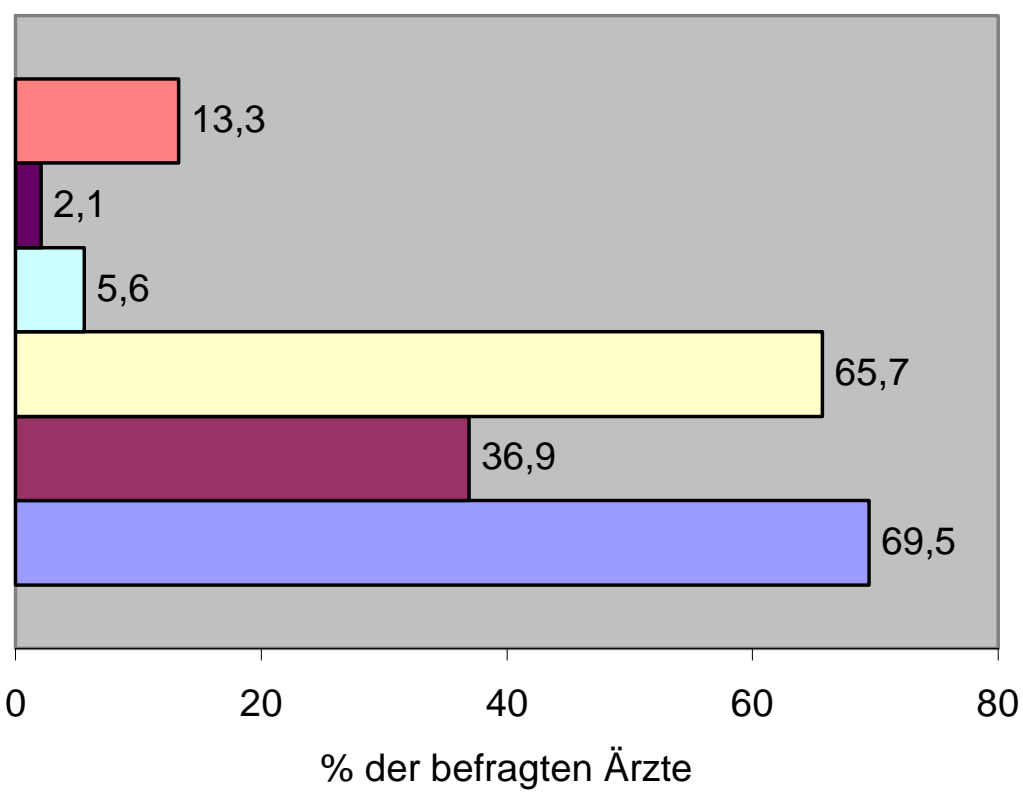

eweggründe zur Durchführung passiver Sterbehilfe ( $n=233$; Mehrfachnennungen mögich) 


\begin{tabular}{|l|}
\hline$\square$ Religiöse \\
Gründe \\
$\square$ Eid des \\
Hippokrates \\
$\square$ Historische Gründe \\
"Drittes Reich" \\
$\square$ Juristische \\
Gründe \\
$\square$ Ausreichende \\
Palliativmedizin \\
$\square$ Möglichkeit ärztlicher \\
Fehleinschätzung \\
$\square$ Möglichkeit des \\
Missbrauchs \\
\hline
\end{tabular}

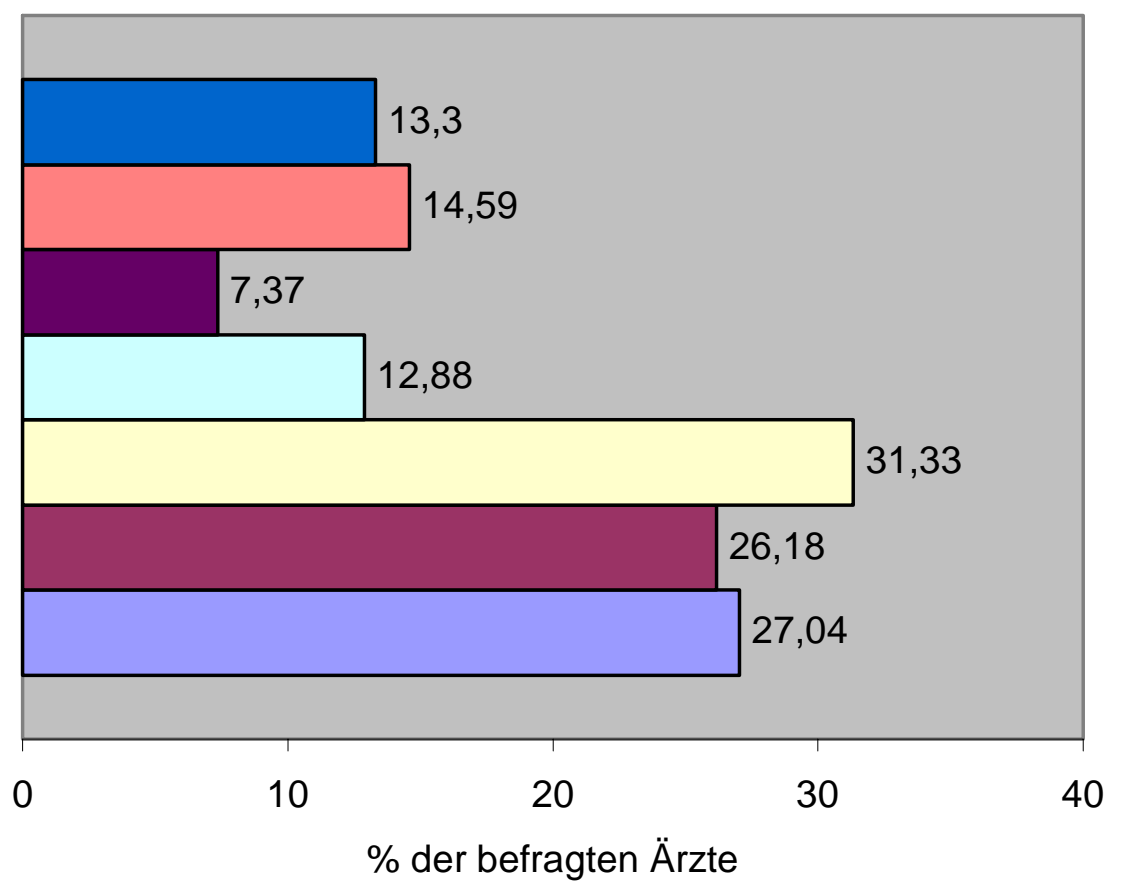

Abbildung 12: Beweggründe gegen die Durchführung der passiven Sterbehilfe ( $n=233$; Mehrfachnennungen möglich)

An Gründen, die die Entscheidung zur Durchführung passiver Sterbehilfe erleichtern könnten, wurde von den Teilnehmern angeführt (Abbildung 13): 
Keine strafrechtliche

Verfolgung

$\square$ Unabhängige Beurteilung durch andere Ärzte

$\square$ Wiederholter Wunsch des

Patienten

$\square$ Schriftlicher Wunsch

des Patienten

Zustimmung der

Angehörigen

$\square$ Ablehnung palliativer Maßnahmen durch den Patienten

$\square$ Ausschluss einer psychischen Erkrankung

$\square$ Volle

Zurechnungsfähigkeit

Degrenzte

Lebenserwartung

$\square$ Nicht beherrschbare

Schmerzen

$\square$ Gute Kenntnis/Lange Betreuung des Patienten

$\square$ Mindestalter des

Patienten

$\square$ Andere Gründe

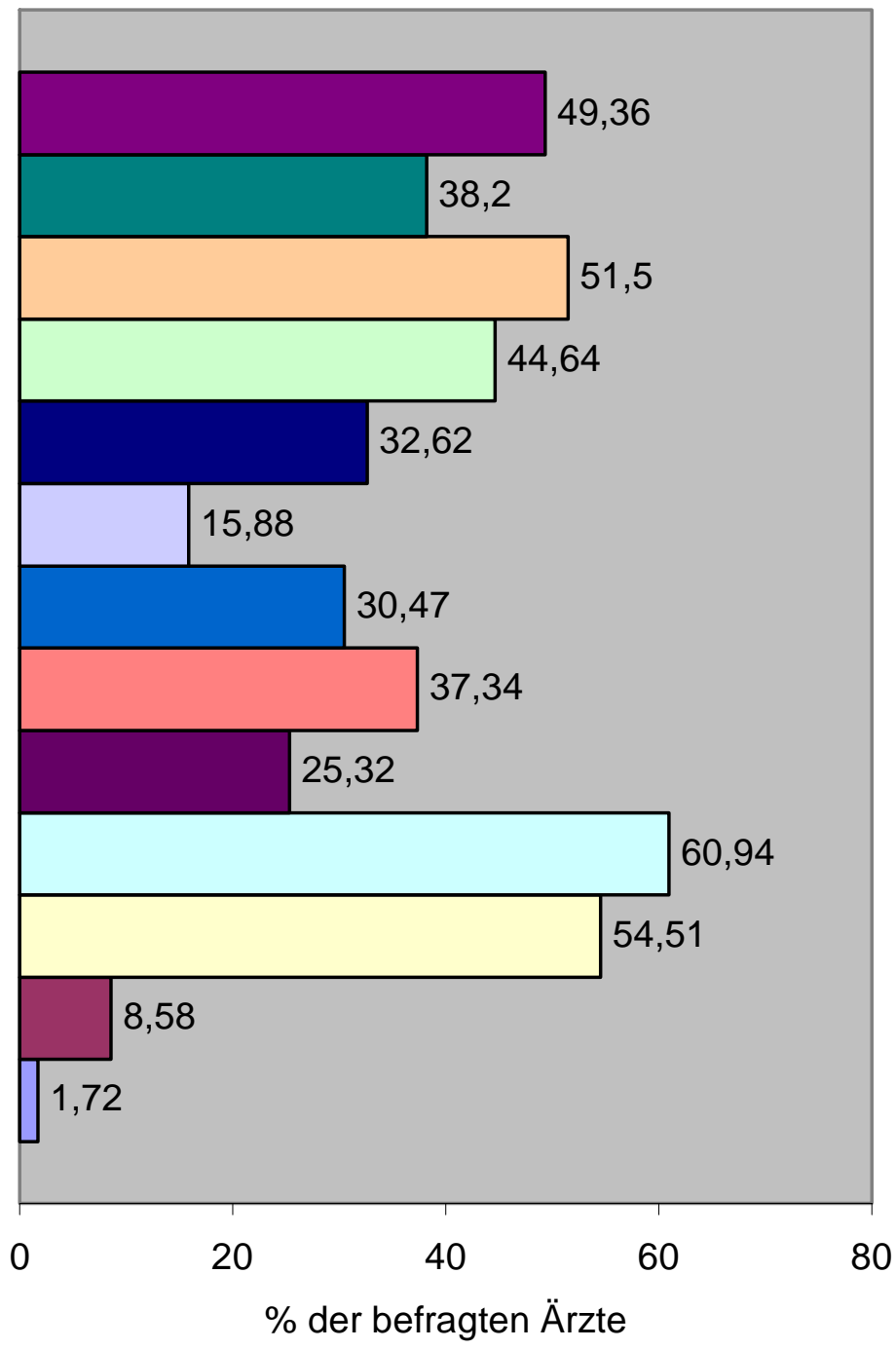

\% der befragten Ärzte

Abbildung 13: Gründe, die die Durchführung von passiver Sterbehilfe erleichtern würden ( $n=233$; Mehrfachnennungen möglich)

Bezüglich der gesetzlichen Regelung gab auch bei der passiven Sterbehilfe die Hälfte der Befragten $(58,74 \%)$ erneut an, dass eine solche eingeführt werden sollte; 4,48\% waren sich hierüber unsicher. Ebenfalls 58,82\% würden passive Sterbehilfe durchführen, wenn eine gesetzliche Grundlage hierfür bestünde; "unsicher" über diesen Sachverhalt war sich jeder Zehnte (12,22\%).

\subsection{Einflüsse auf das Antwortverhalten}

\subsubsection{Allgemeine Beeinflussungsfaktoren}

Ein signifikanter Unterschied in der Akzeptanz von passiver Sterbehilfe ließ sich zwischen Ärzten mit und ohne Kinder aufzeigen. So befürworteten Ärzte mit Kindern passive Sterbehilfe eher als ihre Kollegen ohne Kinder. Bei der Akzeptanz der aktiven Sterbehilfe zeigte sich eine ähnliche Verteilung, allerdings war diese nicht signifikant (Tabelle 4). 


\begin{tabular}{|c|c|c|}
\hline $\begin{array}{ll}\text { Tabelle 4: } & \text { Akzept } \\
& p=0,33 \\
\end{array}$ & $\begin{array}{l}\text { nd passiv } \\
\text { ive Sterbe }\end{array}$ & $\begin{array}{l}\text { mit und ohne Kinder ( } n=216 \text {, } \\
\text { für die passive Sterbehilfe) }\end{array}$ \\
\hline & aktiv & passiv \\
\hline Ärzte mit Kindern & $36,11 \%$ & $83,85 \%$ \\
\hline Ärzte ohne Kinder & $27,78 \%$ & $61,11 \%$ \\
\hline
\end{tabular}

Die Religion und die Intensität ihrer Ausübung war ebenfalls ein wichtiges Moment für die Zustimmung zur Sterbehilfe. Zwischen diesen Parametern konnte folgende Beziehung dargestellt werden (Abbildung 14):

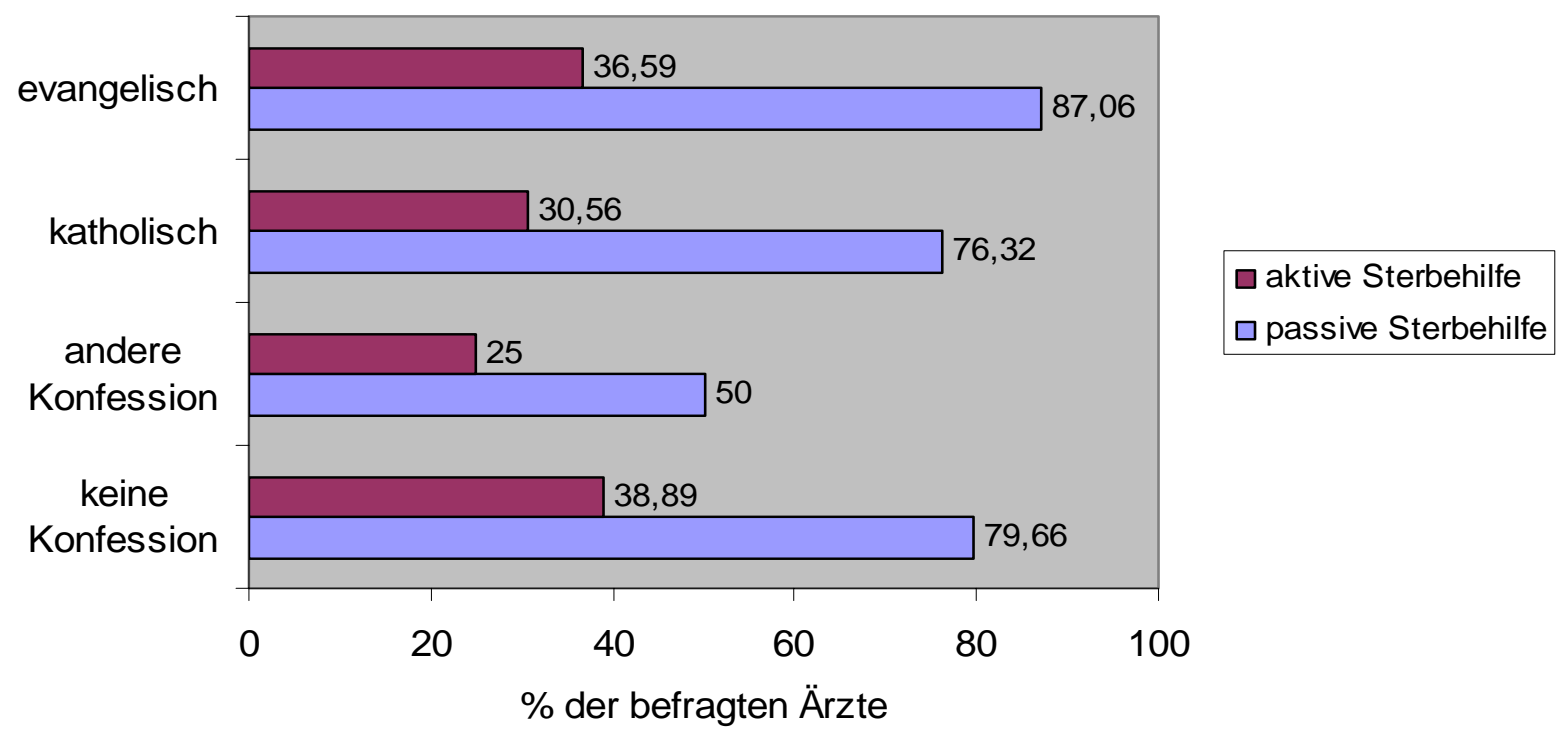

Abbildung 14: Zustimmung zur aktiven und passiven Sterbehilfe in Abhängigkeit von der Konfession $(n=216, p=0,6998$ für die aktive Sterbehilfe; $n=228, p=0,0489$ für die passive Sterbehilfe)

Vor allem religiös aktive Menschen lehnten überwiegend $(47,37 \%)$ jede Form der Sterbehilfe ab $(p=0,0341)$.

Weiterhin konnte aufzeigt werden, dass der bereits erlebte Tätigkeitsbereich der Ärzte einen wichtigen Einfluss auf die Akzeptanz und die Bereitschaft zur Durchführung von Sterbehilfe hatte. Ärzte, die bereits in der Onkologie gearbeitet hatten, waren etwa doppelt so häufig dazu bereit aktive Sterbehilfe durchzuführen als solche, die hier nicht tätig gewesen sind. Ebenso waren bereits onkologisch Tätige signifikant häufiger dazu bereit passive Sterbehilfe durchzuführen (Tabelle 5).

\begin{tabular}{|c|c|c|c|}
\hline \multirow[t]{2}{*}{ Tabelle 5: } & \multicolumn{3}{|c|}{$\begin{array}{l}\text { Bereitschaft zur Durchführung von aktiver und passiver Sterbehilfe in Abhängigkeit von } \\
\text { einer erlebten onkologischen Tätigkeit ( } n=227, p=0,1397 \text { für die aktive Sterbehilfe; } \\
n=227, p=0,0085 \text { für die passive Sterbehilfe) }\end{array}$} \\
\hline & & aktiv & passiv \\
\hline \multicolumn{2}{|c|}{ onkologisch tätig } & $8,75 \%$ & $54,29 \%$ \\
\hline \multicolumn{2}{|c|}{ nicht onkologisch tätig } & $3,82 \%$ & $35,67 \%$ \\
\hline
\end{tabular}


Auch diejenigen Teilnehmer, die bereits in der Geriatrie tätig waren, hielten aktive Sterbehilfe durch Ärzte signifikant häufiger für ein akzeptables Vorgehen, als solche, die auf diesem Gebiet keine Erfahrungen hatten. Eine deutliche Mehrheit derjenigen, die geriatrisch tätig gewesen sind, akzeptierte - ebenso wie ihre nicht geriatrisch orientierten Kollegen - die Durchführung von passiver Sterbehilfe. Über die Hälfte derjenigen, die angaben, vormals geriatrisch gearbeitet zu haben, wünschte sich eine Legalisierung sowohl der aktiven $(56,47 \%, p=0,1694)$ als auch der passiven $(57,14 \%, p=0,9802)$ Sterbehilfe (Tabelle 6).

\begin{tabular}{|c|c|c|}
\hline \multirow[t]{2}{*}{ Tabelle 6: } & \multicolumn{2}{|c|}{$\begin{array}{l}\text { Akzeptanz der aktiven und passiven Sterbehilfe in Abhängigkeit von einer bereits erleb } \\
\text { ten geriatrischen Tätigkeit ( } n=210, p=0,0343 \text { für die aktive Sterbehilfe; } n=223 \text {, } \\
p=0,4498 \text { für die passive Sterbehilfe) }\end{array}$} \\
\hline & aktiv & passiv \\
\hline geriatrisch tätig & $45,53 \%$ & $77,42 \%$ \\
\hline nicht geriatrisch tätig & $28,46 \%$ & $81,54 \%$ \\
\hline
\end{tabular}

Größe und Ort der Praxis (Anzahl der Einwohner) standen in signifikanter Beziehung zur Durchführung von aktiver Sterbehilfe. Ärzte, die in einer Praxis mit bis zu 3.000 Patienten/Quartal arbeiteten, wären eher bereit aktive Sterbehilfe durchzuführen. Dagegen befürwortete dies keiner der Ärzte aus kleinen Praxen bis einschließlich 750 Patienten/Quartal. Auch waren es vor allem Ärzte aus großen Praxen (bis 3.000 Patienten/Quartal), die passive Sterbehilfe durchführen würden (75,00\%). Jedoch auch 40,00\% der Teilnehmer aus kleinen Praxen (Abbildung 15).

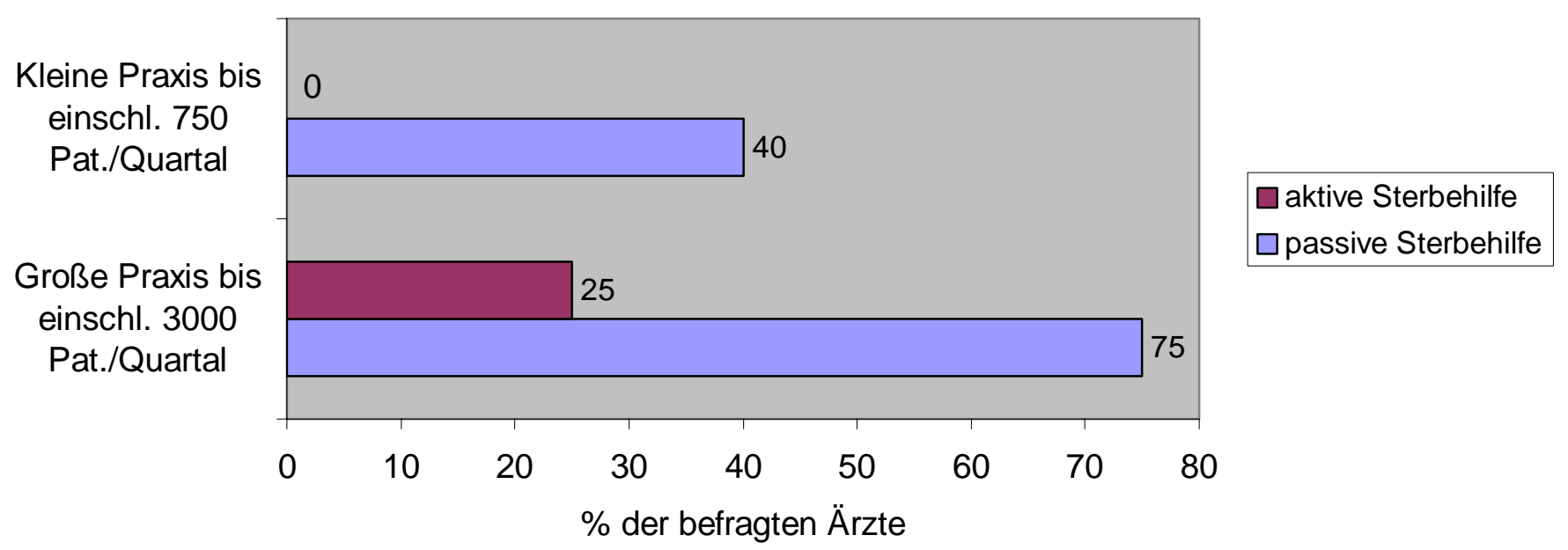

Abbildung 15: Bereitschaft zur Durchführung von aktiver und passiver Sterbehilfe in Abhängigkeit von der Größe der Praxis ( $n=226, p=0,0308$ für die aktive Sterbehilfe; $n=226, p=0,1033$ für die passive Sterbehilfe)

Ebenso wären Ärzte aus kleineren Orten (bis 50.000 Einwohner) eher dazu bereit, aktive Sterbehilfe durchzuführen als solche, die in Großstädten beschäftigt waren. In Orten mit weniger als 50.000 Einwohnern war die Bereitschaft zur Durchführung passiver Sterbehilfe ähnlich hoch wie in größeren Städten ab 51.000 Einwohnern (Tabelle 7). 


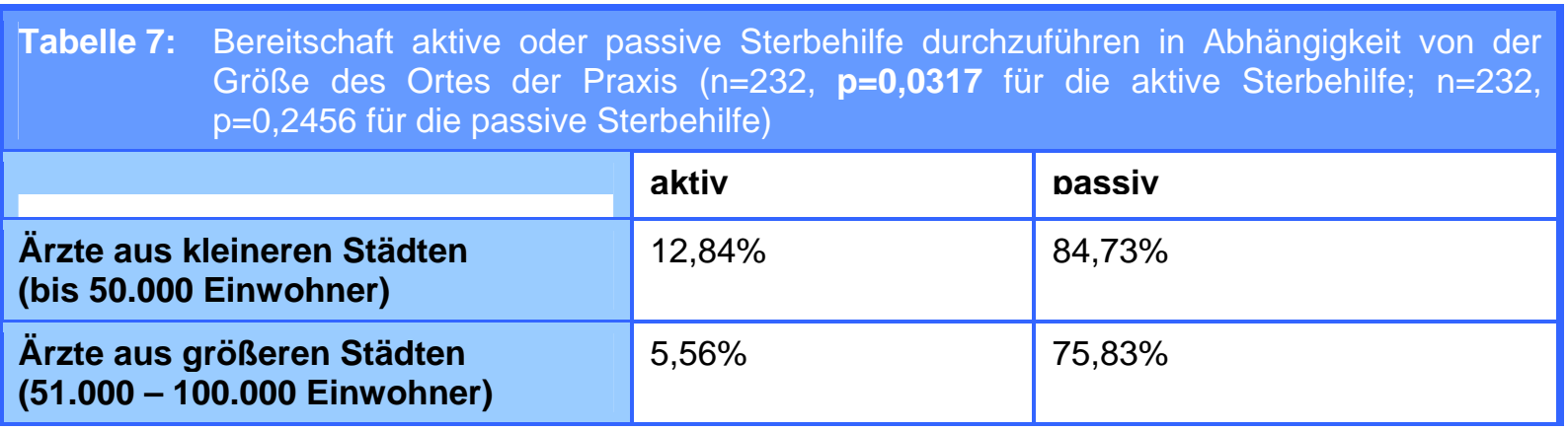

Ein weiterer wichtiger Einflussfaktor für Einstellungen und Handlungsweisen gegenüber der Sterbehilfe war das Jahr der Approbation. So bestand ein signifikanter Zusammenhang zwischen der Dauer der Tätigkeit und der Ablehnung von Sterbehilfe (Abbildung 16).

\begin{tabular}{|l|}
\hline Approbation vor \\
1965 \\
$\square$ Approbation zwischen 1965 \\
und 1982 \\
$\square$ Approbation zwischen 1983 \\
und 2001
\end{tabular}

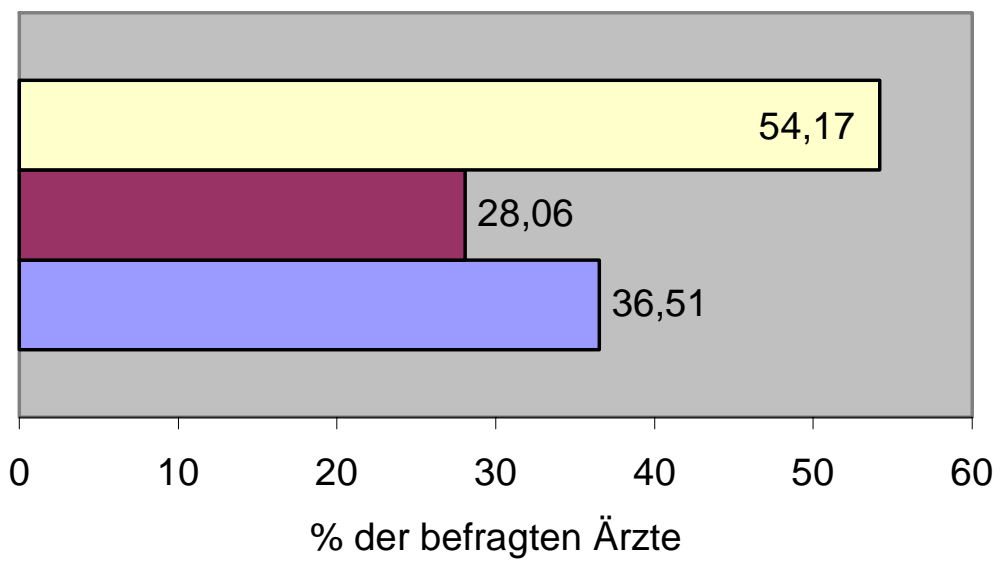

Abbildung 16: Ablehnung der Durchführung von Sterbehilfe in Abhängigkeit vom Jahr der Approbation $(n=226, p=0,0346)$

\subsubsection{Fragen nach Sterbehilfe}

Es existiert eine signifikante Beziehung zwischen der Akzeptanz für die aktive bzw. passive Sterbehilfe und bereits erhaltenen Nachfragen. Jedoch war diese Beziehung nicht abhängig von einer speziellen Anzahl von Bitten nach Sterbehilfe (Abbildung 17). 


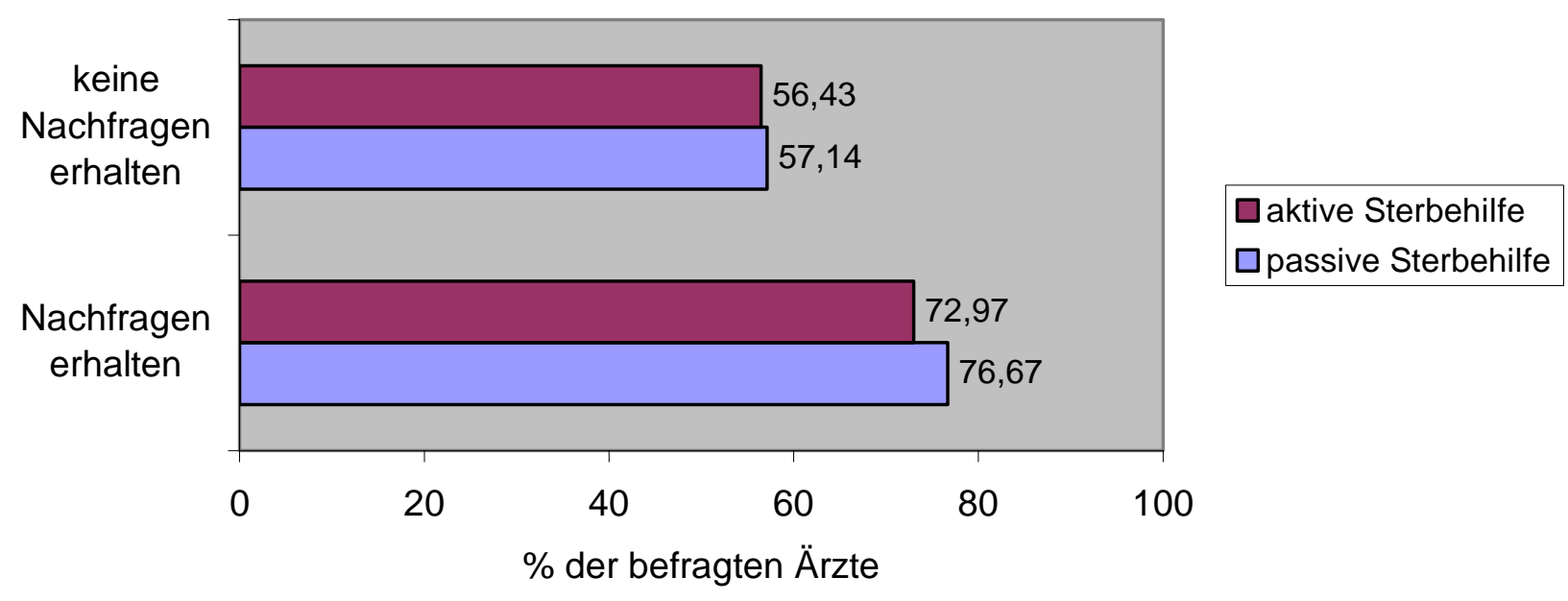

Abbildung 17: Akzeptanz der aktiven Sterbehilfe in Abhängigkeit von erhaltenen Nachfragen ( $n=214$, $p=0,0176$ für die aktive Sterbehilfe; $n=222, p=0,0103$ für die passive Sterbehilfe)

Auch bei der Durchführung der aktiven und passiven Sterbehilfe konnte ebenfalls eine signifikante Abhängigkeit von erhaltenen Nachfragen festgestellt werden (Abbildung 18).

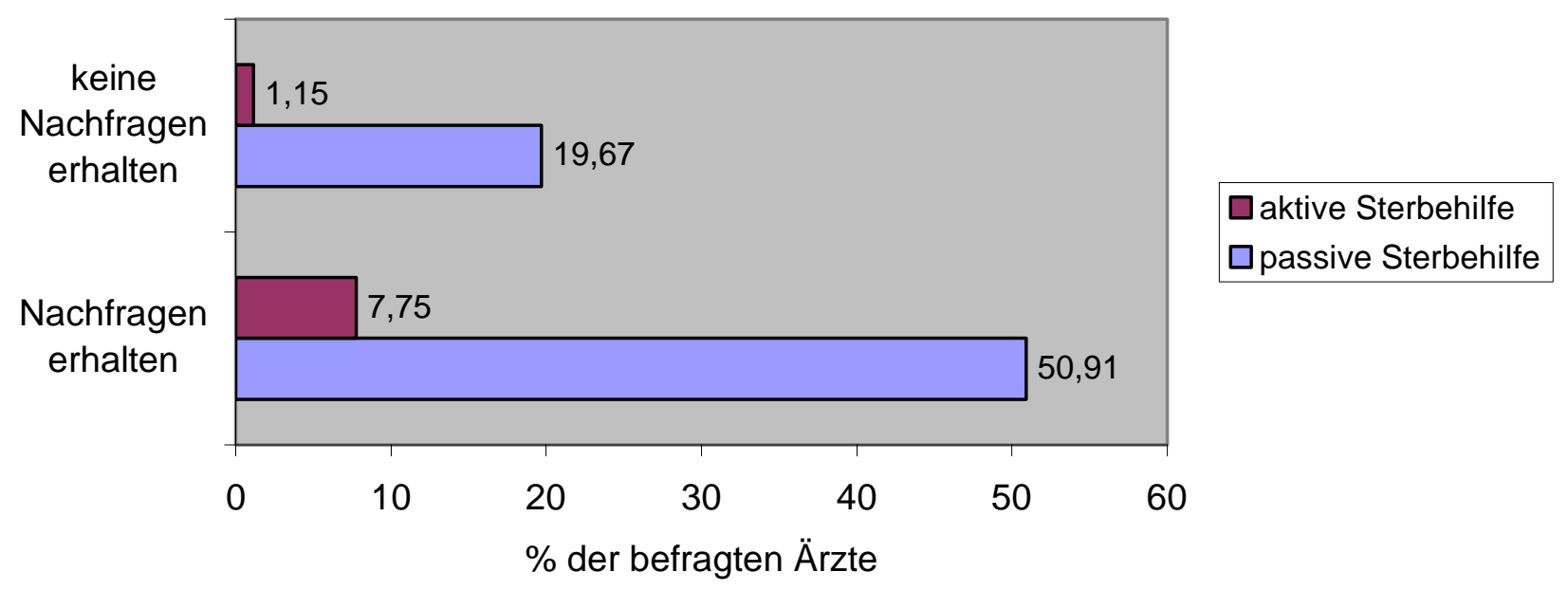

Abbildung 18: Bereitschaft aktive bzw. passive Sterbehilfe durchzuführen in Abhängigkeit von erhaltenen Nachfragen ( $n=229, \mathbf{p}=\mathbf{0 , 0 2 9 7}$ für die aktive Sterbehilfe; $n=226, \mathbf{p}=\mathbf{0 , 0 0 0 1}$ für die passive Sterbehilfe)

\subsubsection{Einfluss von eigener oder bei Angehörigen (mit)erlebte lebensbedrohliche Krankheit}

Hinsichtlich des eigenen Erlebens lebensbedrohlicher Erkrankungen ließen sich keine signifikanten Korrelationen aufweisen: Ärzte, die eine solche Krankheit bereits erlebt hatten, unterschieden sich in der Akzeptanz und der Durchführung von aktiver und passiver Sterbehilfe nicht von Kollegen, die hiervon noch nicht betroffen waren (Abbildung 19). 


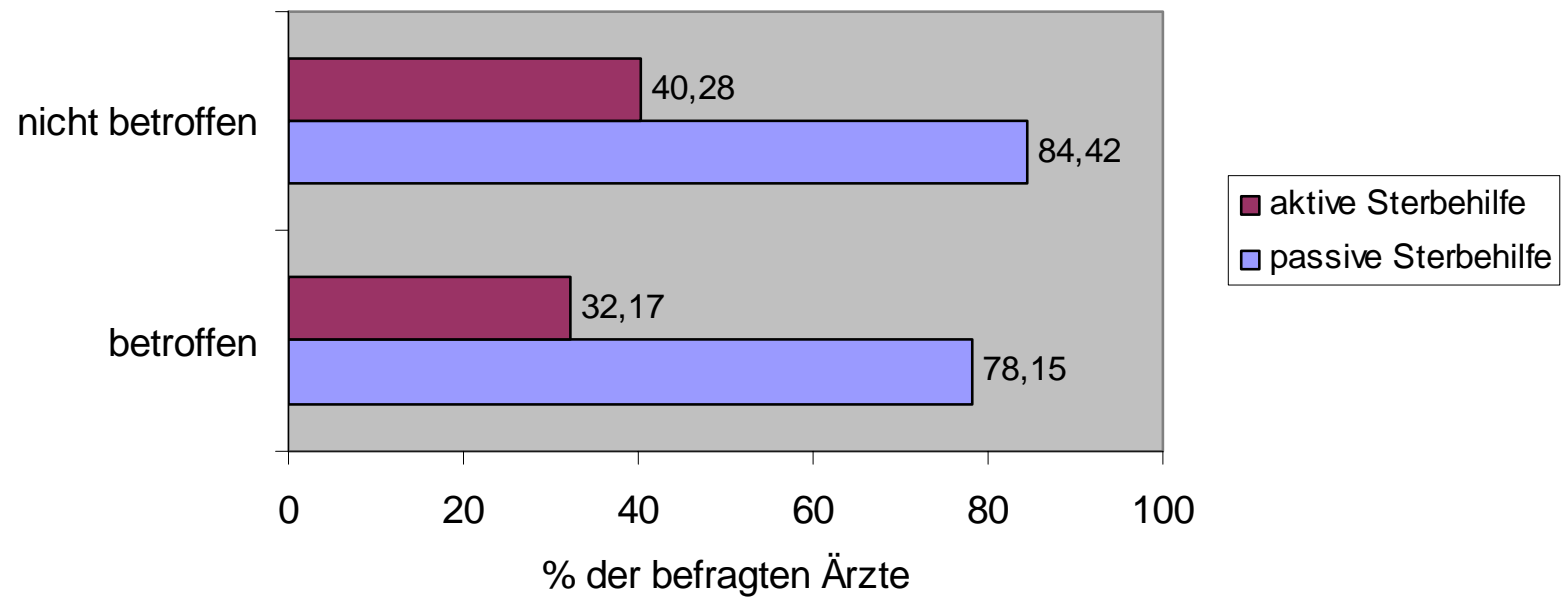

Abbildung 19: Akzeptanz aktiver und passiver Sterbehilfe in Abhängigkeit von einer (mit)erlebten lebensbedrohlichen Krankheit ( $n=215, p=0,2390$ für die aktive Sterbehilfe; $n=228, p=0,2606$ für die passive Sterbehilfe)

Würden betroffene Ärzte von Patientenseite um Sterbehilfe gebeten, unterschieden sie sich in ihren Handlungsweisen ebenfalls nicht von ihren Kollegen (Abbildung 20):

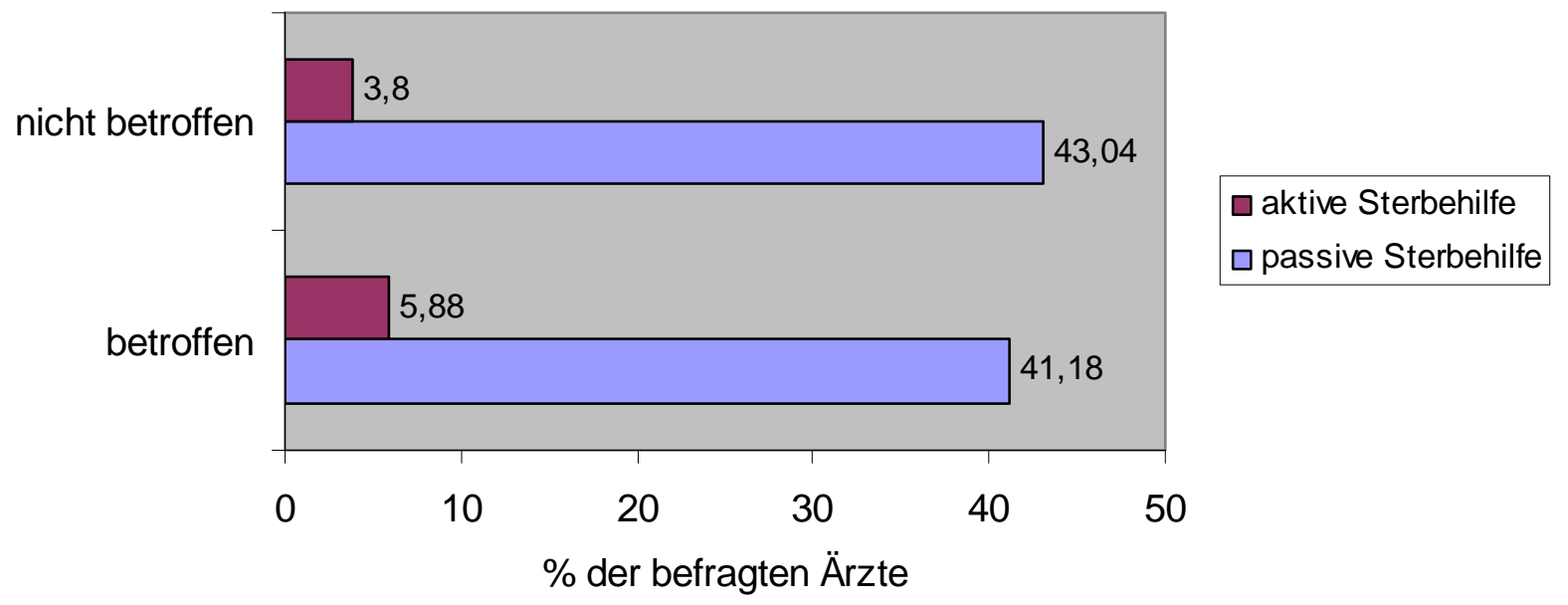

Abbildung 20: Bereitschaft zur Durchführung aktiver und passiver Sterbehilfe in Abhängigkeit von einer (mit)erlebten lebensbedrohlichen Krankheit $(n=232, p=0,4968$ für die aktive Sterbehilfe; $n=232, p=0,7853$ für die passive Sterbehilfe)

\subsubsection{Vergleich zwischen Euthanasie im „Dritten Reich“ und ärztlicher Sterbehilfe}

Bei der Beantwortung von Frage 13 "Halten Sie einen Vergleich zwischen der Euthanasie im sog. "Dritten Reich" und der Durchführung von Sterbehilfe heutzutage für gerechtfertigt?" wurde mit 36,90\% am häufigsten die Möglichkeit eines persönlichen Kommentars genutzt. Hierbei differenzierten die Meinungen zwischen Zustimmung (,Mord ist immer Mord“", „unsere spezielle deutsche Vergangenheit“ und der „Missbrauchsgefahr“) und Ablehnung („zwei grundsätzlich verschiedene Dinge“ und "wir leben heute in einem freien Rechtsstaat und einer Demokratie") dieses Vergleiches. Etwa ein Fünftel der befragten Teilnehmer (20,87\%) empfand einen Vergleich zwischen Euthanasie im sog. „Dritten Reich“ und ärztlicher Sterbehilfe gerechtfertigt. Von diesen Ärzten wa- 
ren fast drei Viertel (73,91\%) nicht an einer gesetzlichen Regelung zur aktiven Sterbehilfe interessiert ( $\mathbf{p}=<\mathbf{0}, \mathbf{0 0 0 1})$; etwa die Hälfte $(53,33 \%)$ hielt eine gesetzliche Regelung für die passive Sterbehilfe ebenfalls für nicht erforderlich $(\mathbf{p}=\mathbf{0}, \mathbf{0 1 9 7})$. Nach ihrer Meinung befragt, akzeptierte nur ein kleiner Teil der Ärzte, die dem oben genannten Vergleich zustimmten, aktive Sterbehilfe durch Ärzte. Der überwiegende Teil hingegen stufte die Durchführung passiver Sterbehilfe durch Ärzte für akzeptabel ein (Abbildung 21).

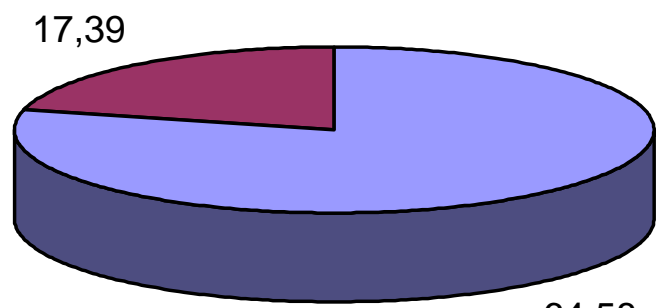

Akzeptanz der passiven Sterbehilfe

Akzeptanz der aktiven Sterbehilfe

$\%$ der befragten Ärzte

Abbildung 21: Akzeptanz der aktiven und passiven Sterbehilfe bei Ärzten, die einen Vergleich zwischen der Euthanasie im sog. "Dritten Reich" und der heutigen Sterbehilfe für gerechtfertigt halten $(n=213, p=0,0043$ für die aktive Sterbehilfe; $n=226, p=0,0017$ für die passive Sterbehilfe)

Statistisch nicht signifikant, aber erwähnenswert ist die Tatsache, dass für die Ärzte, die eine Verbindung zum sog. "Dritten Reich" gezogen haben, eine Legalisierung der Sterbehilfe ihr Verhalten ändern würde: Die Bereitschaft derjenigen, die in diesem Falle bereit wären aktive Sterbehilfe durchzuführen, wäre mehr als doppelt so hoch gewesen. Auf die Durchführung passiver Sterbehilfe hätte eine Legalisierung hingegen nur geringen Einfluss gehabt (Abbildung 22). 


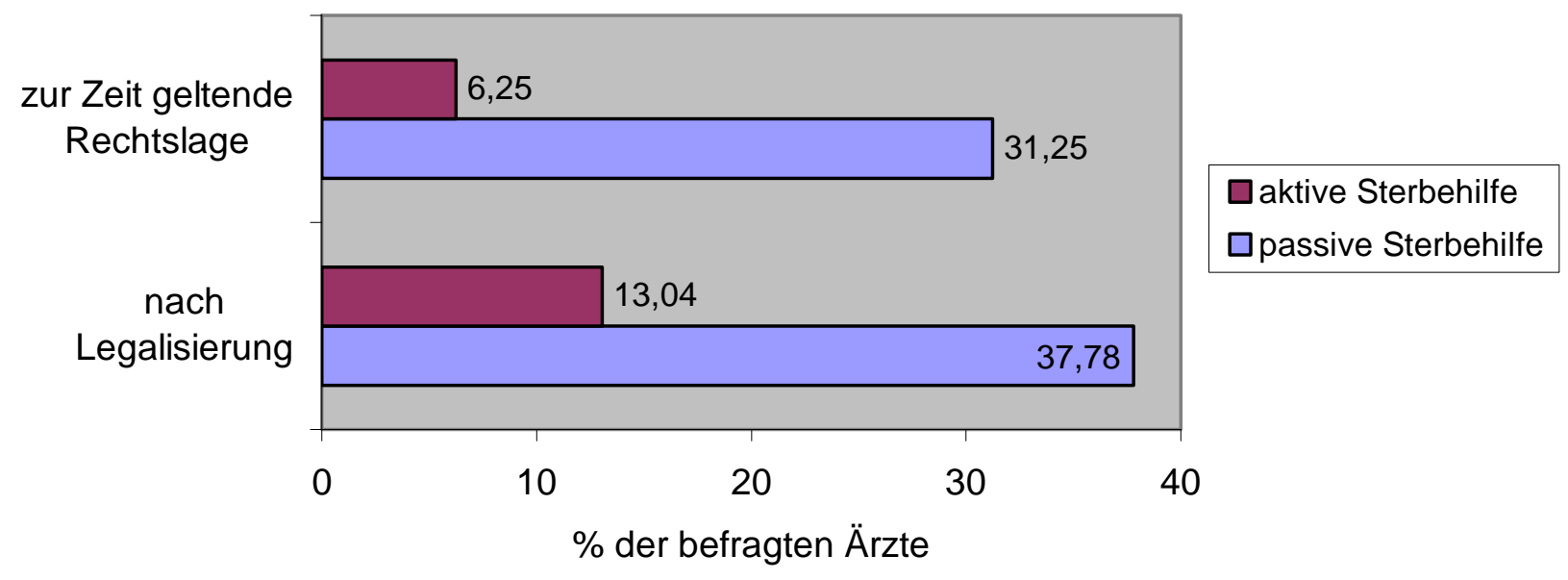

\begin{abstract}
Abbildung 22: Bereitschaft zur Durchführung aktiver und passiver Sterbehilfe ${ }^{15}$ in Abhängigkeit individueller gesetzlicher Voraussetzungen ( $n=230, p=0,0901$ für die aktive Sterbehilfe in der zur Zeit geltenden Rechtslage; $n=230, p=0,0849$ für die passive Sterbehilfe in der zur Zeit geltenden Rechtslage; $n=206, p=0,7176$ für die aktive Sterbehilfe im Falle einer Legalisierung; $\mathrm{n}=219, \mathbf{p}=\mathbf{0 , 0 0 4 2}$ für die passive Sterbehilfe im Falle einer Legalisierung)
\end{abstract}

\title{
5.5.5 Beschäftigung mit den Themen "Sterben" und "Sterbehilfe" während der me- dizinischen Ausbildung
}

Die Beschäftigung während der Ausbildung mit den Themen Sterben und Sterbehilfe stand in keiner signifikanten Beziehung zur Durchführung von Sterbehilfe oder zu den Wünschen für oder gegen eine Legalisierung. Sie war jedoch signifikant in der Einstellung zur (passiven) Sterbehilfe: So lehnten Ärzte, die sich mit der Thematik schon in der Ausbildung auseinandergesetzt hatten, passive und aktive Sterbehilfe eher ab als ihre Kollegen (Abbildung 23).

\footnotetext{
${ }^{15}$ Gilt für diejenigen Ärzte, die einen Vergleich zwischen Euthanasie im sog. "Dritten Reich" und ärztlicher Sterbehilfe für gerechtfertigt halten.
} 


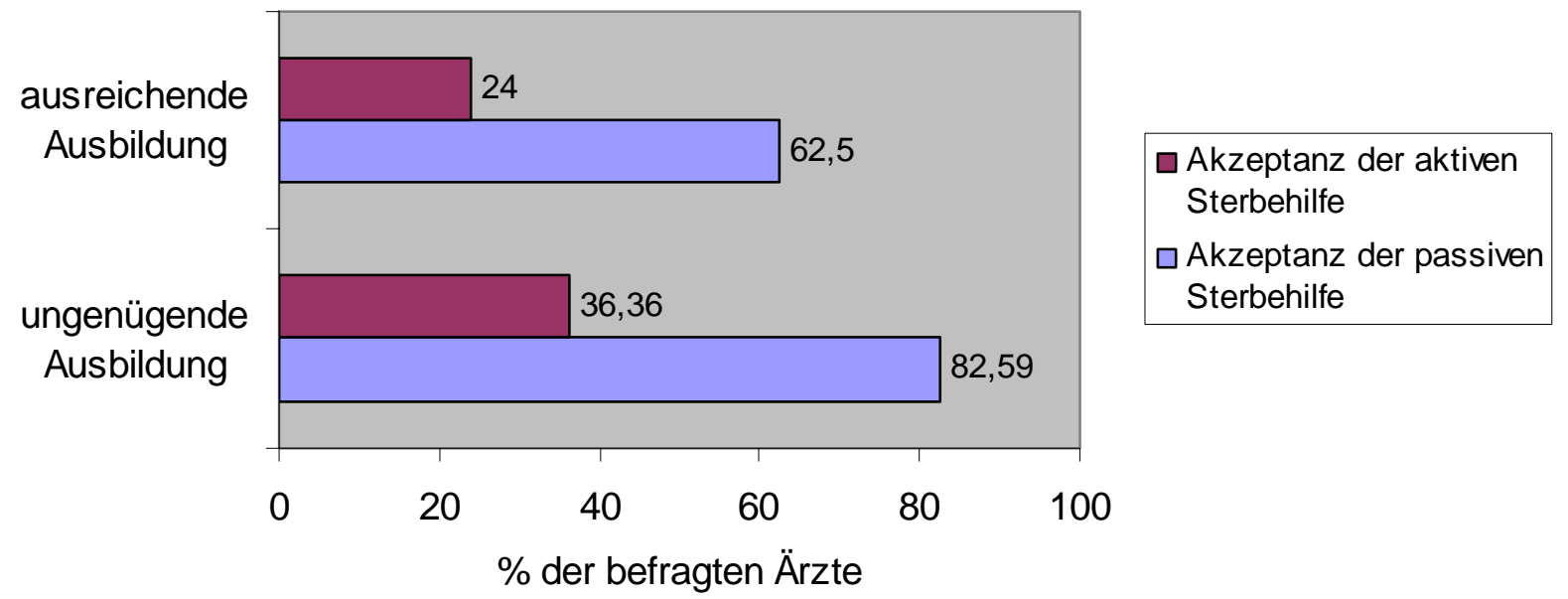
Abbildung 23: Einfluss der Beschäftigung mit den Themen "Sterben" und "Sterbehilfe" während der Aus- bildung auf die Akzeptanz aktiver und passiver Sterbehilfe ( $n=212, p=0,2232$ für die aktive Sterbehilfe; $n=225, \mathbf{p}=\mathbf{0 , 0 1 9 0}$ für die passive Sterbehilfe)

\subsubsection{Beziehung zwischen Legalisierung und Durchführung von Sterbehilfe}

Eine Legalisierung der Sterbehilfe würde das Verhalten der Ärzte, die sich vormals explizit gegen die Durchführung von aktiver oder passiver Sterbehilfe aussprachen, signifikant ändern. Jeweils ein Viertel erklärte sich unter neuen gesetzlichen Richtlinien bereit, Sterbehilfe durchzuführen (Abbildung 24).

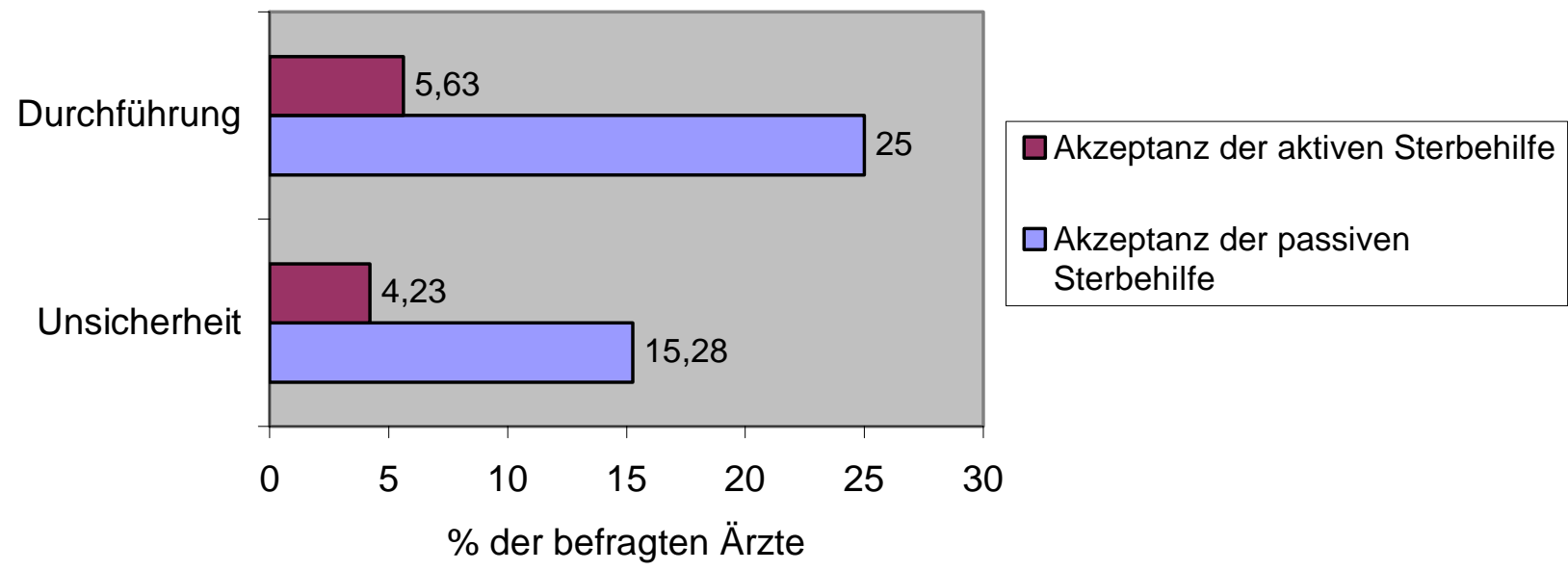

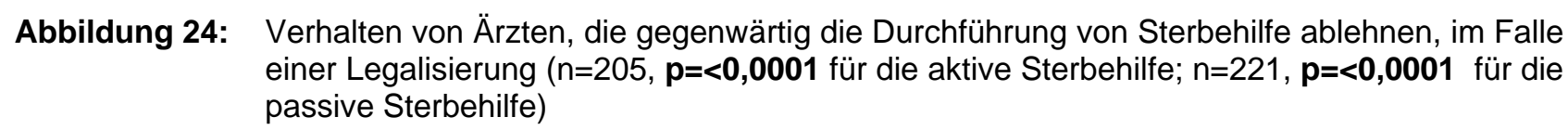
Wie entscheidend der Status der Illegalität für die Haltung zur Sterbehilfe bei Ärzten ist, zeigt sich auch daran, dass die Freiheit vor strafrechtlicher Verfolgung als Beweggrund für die mögliche Durchführung aktiver Sterbehilfe (69,33\%) - unmittelbar nach der Motivation den Patienten von nicht beherrschbaren Schmerzen (72,00\%) zu erlösen - genannt wurde. 
Bei der passiven Sterbehilfe war dieses Bild nicht ganz so einheitlich: Strafrechtliche Verfolgung war hier zwar auch eine wichtige Vorbedingung (60,33\%), doch standen hier vor allem eine gute bzw. lange Betreuung des Patienten (65,76\%), sowie nicht beherrschbare Schmerzen (74,46\%) im Vordergrund. Einen weiteren Hinweis auf die außerordentliche Bedeutung einer strafrechtlichen Verfolgung ergab sich durch den Vergleich der Aussagen in den Fallvignetten (angenommene Legalität von Sterbehilfe) und den Antworten, die im weiteren Fragebogen getätigt wurden (tatsächliche Illegalität): So würden in der momentan juristisch geltenden Situation der Bundesrepublik von den Befürwortern der aktiven Sterbehilfe nur maximal die Hälfte diese ebenfalls durchführen. Bei der passiven Sterbehilfe hingegen zeigte sich diesbezüglich ein einheitliches Bild: Würde die passive Sterbehilfe legalisiert werden, wäre fast jeder, der in den Fallvignetten der passiven Sterbehilfe zustimmte, dazu bereit gewesen, dies auch in einer realen Situation unter der momentanen Gesetzeslage zu tun (Abbildung 25).

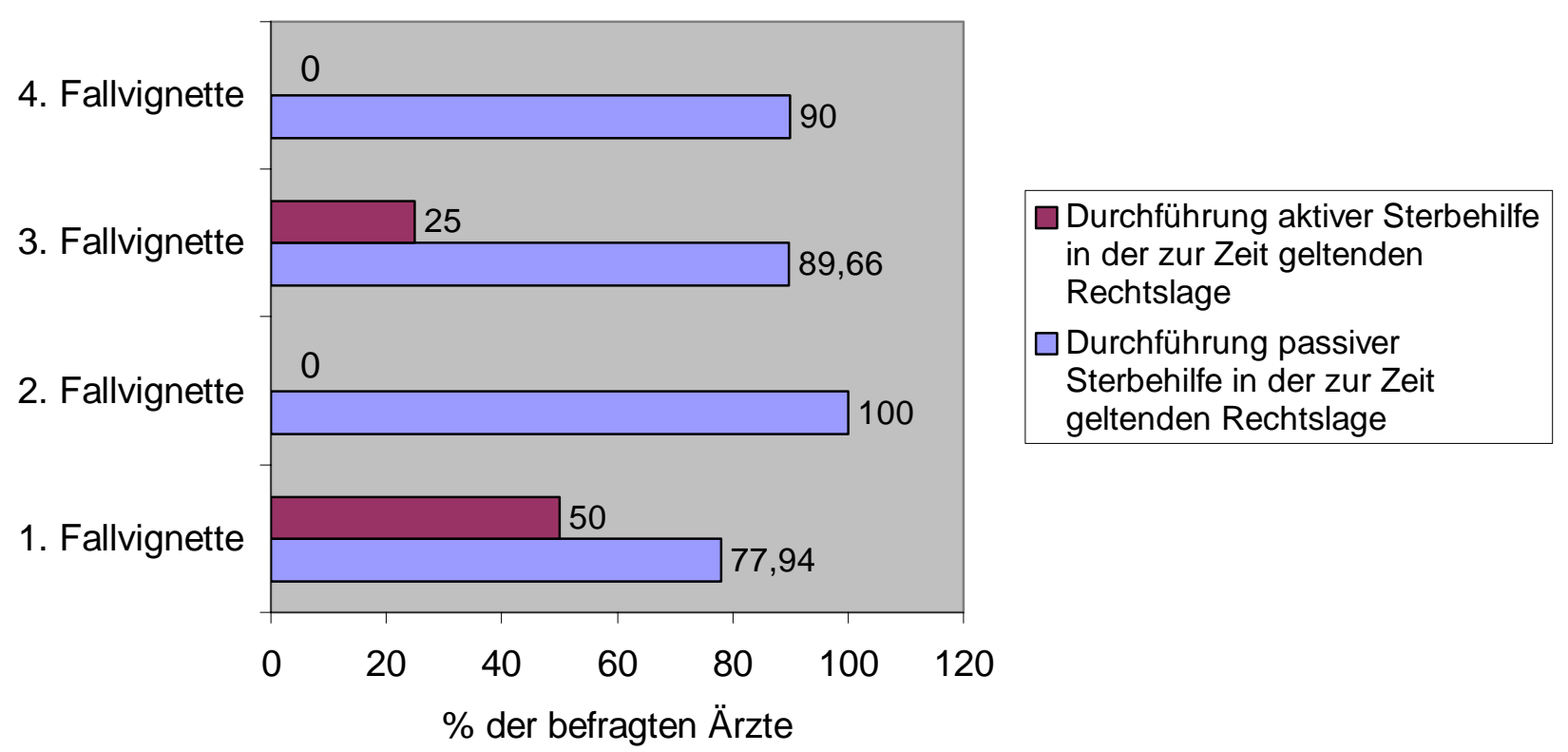

Abbildung 25: Bereitschaft zur Durchführung aktiver bzw. passiver Sterbehilfe in der zur Zeit geltenden Rechtslage bezogen auf die einzelnen Fallvignetten ${ }^{16}$ (1. Fallvignette $n=225,2$. Fallvignette $n=226$, 3. Fallvignette $n=227$, 4, Fallvignette $n=229$; für die aktive Sterbehilfe: 1 . Fallvignette $\mathbf{p}=<\mathbf{0 , 0 0 0 1}, 2$. Fallvignette $p=0,8156,3$. Fallvignette $\mathbf{p}=\mathbf{0 , 0 2 4 4}, \mathbf{4}$. Fallvignette $p=0,0920$; für die passive Sterbehilfe: 1 . Fallvignette $p=<0,0001,2$. Fallvignette $p=<0,0001,3$. Fallvignette $p=<0,0001,4$. Fallvignette $p=0,0020$ )

\subsection{Logistische Regression}

Die logistische Regression wurde anhand von 170 Beobachtungen über Einstellungen zur aktiven Sterbehilfe bzw. von 173 Beobachtungen für die passive Sterbehilfe berechnet. 63 Beobachtungen

\footnotetext{
${ }^{16}$ Berücksichtigt wurden nur diejenigen Ärzte, die in den Fallvignetten angaben, aktive bzw. passive Sterbehilfe leisten zu wollen
} 
zur aktiven (bzw. 60 zur passiven) Sterbehilfe wurden aufgrund fehlender Werte gestrichen. Es konnte kein Einfluss des Alters, Geschlechts, Familienstandes oder der Konfession auf die Einstellung zur passiven Sterbehilfe festgestellt werden. Ferner beeinflussten auch Erfahrungen in der Onkologie oder Geriatrie und das (Mit)erleben einer lebensbedrohlichen Krankheit die Einstellung der Ärzte bezüglich der passiven Sterbehilfe nicht. Diejenigen Ärzte jedoch, die eigene Kinder hatten, waren mehr als vier Mal häufiger bereit, passive Sterbehilfe durchzuführen, als Kollegen ohne Kinder (OR= 4.59; (1.77-11.95)).

Ärzte, die bereits Nachfragen nach passiver Sterbehilfe erhalten hatten, waren fast drei Mal so häufig bereit, passive Sterbehilfe durchzuführen als vergleichsweise solche, die noch keine Nachfragen erhalten hatten $(\mathrm{OR}=2.87$; (1.22-6.73)). Je aktiver die Ärzte angaben in ihrer jeweiligen $\mathrm{Re}$ ligionsausübung zu sein (Antwortmöglichkeit: aktiv- gelegentlich aktiv- nicht aktiv), desto seltener akzeptierten sie die Durchführung passiver Sterbehilfe (OR=0.581; (0.345-0.979)).

Die gleichen abhängigen Variablen wurden in einem Model für die Einstellung gegenüber aktiver Sterbehilfe verwendet. Hierbei zeigte sich ein signifikanter Zusammenhang: Die Ärzte, die bereits Nachfragen nach aktiver Sterbehilfe erhalten hatten, waren zwei Mal so häufig dazu bereit, als Kollegen, die noch keine Nachfragen erhalten hatten (OR=2.36; (1.18- 4.74)).

\section{Diskussion}

\subsection{Methode}

Die vorliegende Arbeit war die erste randomisierte und bundesweit durchgeführte Studie, die speziell die Haltung von Allgemeinmedizinern zum Thema Sterbehilfe untersuchte. Sie gab ferner Daten zur Häufigkeit von Nachfragen und zur Durchführung von aktiver und passiver Sterbehilfe im hausärztlichen und familienmedizinischen Bereich. Die Position von Allgemein- und Hausärzten ist aufgrund ihrer besonderen Tätigkeit notwendigerweise eine andere als bspw. die von Onkologen oder Psychiatern. Die Betreuung Sterbender in der häuslichen Umgebung ist ein Bereich, der vor allem Allgemein- und Hausärzte betrifft (Abholz 1998). Bewusst stützte sich diese Untersuchung nur auf die Haltung von Allgemeinärzten zu dem Thema, da andernfalls eine differenzierte Betrachtung kaum möglich erschien. Durch die in den meisten internationalen Studien vorgenommene Vermengung von Meinungen verschiedener Fachrichtungen war ein Vergleich von nationaler und internationaler Ebene zwischen Positionen verschiedener Ärztegruppen nicht möglich.

Individuelle Haltungen zu ethisch problembehafteten Themenbereichen wie der Sterbehilfe sind nur vor dem Hintergrund der persönlichen Situation und Tätigkeit der teilnehmenden Ärzte zu verstehen. Daher wurde im Gegensatz zu den bisher in Deutschland durchgeführten Studien in der vorliegenden Untersuchung Wert auf die Möglichkeit einer systematischen Auswertung der Ein- 
flussfaktoren der Befürwortung oder Ablehnung von Sterbehilfe gelegt, die einen differenzierten Überblick über die Einstellungen und Verhaltensweisen zulässt.

In der Studie von Kirschner und Elkeles (Kirschner und Elkeles 1998) konnte gezeigt werden, dass zwischen mündlicher und schriftlicher Erhebung keine Unterschiede auftraten. Daher wurde als praktikabelste Möglichkeit für diese Untersuchung ein Fragebogen entwickelt, der an zufällig ausgewählte Ärzte geschickt wurde.

US- amerikanische Studien zur Sterbehilfe konnten ferner aufzeigen, dass die Gewährleistung der Anonymität von besonderer Bedeutung für Untersuchungen zur Sterbehilfe ist, da über etwas "partiell Illegales" berichtet werden sollte. Sahen die angeschriebenen Ärzte ihre Anonymität gefährdet, sandten sie ihre Fragebögen nicht oder nur unvollständig zurück (Emanuel et al. 1996). Daher kann vermutet werden, dass auch die Resonanz auf den Fragebogen in der vorliegenden Arbeit noch besser gewesen wäre, wenn die Ärzte nicht befürchten müssten über partiell illegale Handlungen zu berichten. Den Teilnehmern wurde deshalb in einem beiliegenden Anschreiben die vollständige Anonymisierung zugesichert. Rückschlüsse auf die antwortende Person waren durch die Verwendung namentlich nicht gekennzeichneter und nicht nummerierter Fragebögen unmöglich. Durch dieses Vorgehen war andererseits die Möglichkeit des gezielten Erinnerns einzelner Teilnehmer, die möglicherweise den Recall erhöht hätten, eingeschränkt. Aus logistischen Gründen wurde sich insofern auf ein Recallschreiben beschränkt, das an alle potentiellen Teilnehmer ausgesandt wurde.

Probleme, die bei der Beantwortung der Frage nach (aktiver) Sterbehilfe auftraten, betrafen die Abgrenzung der Begriffe aktiver und passiver Sterbehilfe. Die meisten Ärzte hatten eine eigene und sehr subjektive Vorstellung davon, was unter „Sterbehilfe“ zu verstehen ist. Allgemein akzeptierte Definitionen hierzu existierten nicht (Beauchamp und Davidson 1979). Es zeigte sich vielmehr, dass gerade in Grenzfällen die Vorstellungen der Kennzeichen und Charakteristika von „aktiver“ und „passiver“ Sterbehilfe variieren, oder gar divergent sind (Quante 1998). Um eine einheitliche Begrifflichkeit zu gewährleisten, wurden die angeschriebenen Ärzte gebeten, für die Beantwortung des Fragebogens die vorgegebenen Definitionen zugrunde zu legen. Die geschilderten Schwierigkeiten einer einheitlichen Definition von Sterbehilfe erschwerten den Vergleich mit internationalen Untersuchungen zusätzlich. Die diffizile Trennung von „aktiver“ und „passiver“ oder auch „direkter" und „indirekter" Sterbehilfe ist eine nahezu ausschließlich in Deutschland getroffene Unterscheidung von oftmals geringer Trennschärfe, die vielfach auf Widerstand stößt (Klaschik 1999). Die hingegen im angelsächsischen Sprachraum gebräuchlichen Definitionen von „euthanasia“ oder „physician- assisted - suicide“ finden jedoch in Deutschland keine Entsprechung. Der Begriff der Euthanasie ist in Deutschland vor allem für die Beschreibung von Verbrechen während des "Dritten Reichs" gebräuchlich. Die in der vorliegenden Arbeit gewählten Definitionen wurden in 
Anlehnung an deutsche und internationale Publikationen und in Absprache mit der Abteilung Medizinische Ethik der Universität Göttingen generiert. Aufgrund der hohen Verunsicherung der deutschen Ärzte zu diesen Definitionen und zum Umgang mit dem Thema Sterbehilfe im Allgemeinen (Sohn 2002) wünschten sich über die Hälfte der Ärzte (50,8\%) mehr Diskussion zum Thema (Kirschner und Elkeles 1998). Dies kam der vorliegenden Studie zu Gute, was daraus ersichtlich ist, dass die Antwortrate mit 48,44\% über der vergleichbarer anderer internationaler Studien lag (bspw.: Kuhse und Singer 1988, Mc Glade et al. 2000, Siaw 1996).

\subsection{Durchführung von aktiver Sterbehilfe}

In der vorliegenden Studie gab etwa die Hälfte der Teilnehmer an, bereits Nachfragen nach aktiver Sterbehilfe erhalten zu haben. Bereits jeder zehnte hatte schon einmal aktive Sterbehilfe durchgeführt. Ca. jeder fünfte wäre dazu bereit, sofern es eine gesetzliche Regelung zur Sterbehilfe gäbe.

Auf die tatsächliche Häufigkeit der Durchführung aktiver Sterbehilfe konnte indirekt geschlossen werden. Wurde die Frage: "Falls Sie jemals Sterbehilfe durchgeführt haben, glauben Sie auch rückblickend, das Richtige getan zu haben" mit "ja" oder "unsicher" beantwortet, wurde davon ausgegangen, dass die Ärzte bereits aktive bzw. passive Sterbehilfe durchgeführt hatten. Bewusst wurde auf eine direkte Nachfrage nach der Durchführung von Sterbehilfe verzichtet, da anderenfalls ein Verzerrungsfehler zum Tragen gekommen wäre: Da es sich bei der Durchführung aktiver Sterbehilfe nach derzeit gültiger Rechtsprechung um eine illegale Handlung handelt, ist anzunehmen, dass vor allem Ärzte zur Beantwortung der Fragen bereit waren, die Sterbehilfe ohnehin ablehnten bzw. Antworten im Sinne des Gesetzes gegeben werden, um mögliche rechtliche Konsequenzen zu vermeiden. Es kann insofern ohnehin von einer überrepräsentativen Darstellung ablehnender Haltungen gegenüber Fragen zur aktiven Sterbehilfe ausgegangen werden. Problematisch stellte sich hierdurch die indirekte Bewertung dieser Frage dar. Es konnte nicht mit Sicherheit davon ausgegangen werden, dass die positive Beantwortung dieser Frage oder ein "unsicher" als Antwortmöglichkeit direkt mit der Durchführung von aktiver/passiver Sterbehilfe korreliert ist. Jedoch erschien diese indirekte Art der Auswertung als praktikabelste Möglichkeit, Tendenzen zur Durchführung von Sterbehilfe heraus zu arbeiten, ohne hierbei den Anspruch absoluter Korrektheit des Verfahrens erheben zu wollen. Ein Beleg des angenommenen Verzerrungsfehlers wäre prinzipiell durch eine "Non-responder" zu erbringen gewesen, was aber aufgrund des Studiendesigns mit der Maxime nach Anonymität nicht durchführbar war. ${ }^{17}$

Die vorliegende Studie konnte aufzeigen, dass Sterbehilfe - trotz derzeitiger gesetzlicher Sanktionierungen - bereits von vielen Ärzten praktiziert worden ist.

\footnotetext{
${ }^{17}$ Zum Beleg für diese Überlegungen sei hier nur am Rande berichtet, dass ein Arzt seine Nichtteilnahme an dieser Studie damit erläuterte, dass er trotz der gegebenen Anonymisierung befürchtete, anhand seiner Fingerabdrücke auf dem Fragebogen reidentifiziert zu werden.
} 
Es herrschte jedoch eine große Diskrepanz zwischen der relativ hohen Anzahl an Nachfragen einerseits und der seltenen Durchführung andererseits. Dies kann zunächst durch die juristischen Vorgaben begründet werden: Etwa ein Viertel (24,82\%) derjenigen Ärzte, die unter der jetzigen Gesetzeslage die Durchführung von Sterbehilfe generell ablehnten, würden diese bei einer entsprechenden Gesetzesänderung durchführen. Die Hälfte $(56,20 \%)$ würde auch unter der Annahme gesetzlicher Vorgaben bei ihrer Ablehnung bleiben. Übereinstimmend mit der vorliegenden Studie konnte auch in der 1998 erschienen Berliner Studie von Kirschner und Elkeles festgestellt werden, dass "etwa die Hälfte aller Ärzte in Deutschland (...) um aktive Sterbehilfe gebeten worden ist" (Kirschner und Elkeles 1998, S. 249), wobei sich der Fragemodus bezüglich Fragen zur aktiven Sterbehilfe unterscheidet. In dieser Studie bspw. wurde nicht nur direkt nach Nachfragen, sondern ebenfalls auch direkt nach der Durchführung von Sterbehilfe gefragt. Durch dieses Studiendesign werden Ärzte vermutlich noch seltener über eine eventuell illegale Handlung berichten - was also eine zusätzliche Überrepräsentation ablehnender Haltungen zur Folge gehabt haben wird. ${ }^{18}$

Auch mehrere internationale Studien konnten darstellen, dass etwa ein Viertel (28\%, Baume und O' Malley 1994) bis fast die Hälfte (44\%, Baume und O' Malley 1996) der befragten Ärzte bereits aktive Sterbehilfe durchgeführt hatte. Aufgrund der unterschiedlichen Stichproben bzw. Definitionen war ein Vergleich der einzelnen Ergebnisse allerdings problematisch.

\subsection{Gründe für das Durchführen von aktiver Sterbehilfe}

Die befragten Allgemeinärzte gaben als hauptsächliche Beweggründe gegen die Durchführung von aktiver Sterbehilfe die Möglichkeit eines Missbrauchs $(34,4 \%)$ und die Gefahr der ärztlichen Fehleinschätzung (31,3\%) an. Das Hauptmotiv einer Befürwortung von aktiver Sterbehilfe ergab sich durch eine Erlösung des Patienten von Schmerzen (27,9\%). Auffällig zeigte sich hierbei, dass sich die Argumentation überwiegend auf einer Ebene bewegte, in der die ärztliche Entscheidung und Beurteilung im Vordergrund stand. Patientenzentrierte Gründe, wie bspw. das Recht auf einen selbstbestimmten Tod, wurden deutlich seltener (14,6\%) benannt. Einige der Ärzte äußerten Probleme bei der Beantwortung dieser Frage, bei der Gründe benannt werden sollten, die zur individuellen Einstellung in Bezug zur aktiven bzw. passiven Sterbehilfe beigetragen haben. Einige Ärzte benannten hier jedoch nur Gründe für die Form der Sterbehilfe, der sie auch zustimmten, nicht aber für die Form, die sie ablehnten. Somit wurden quantitativ häufiger nur Gründe bei der passiven Sterbehilfe angekreuzt. Handlungsmotivation für die Ärzte, die für die aktive Sterbehilfe plädierten, waren primär Gründe auf die sie selbst von außen Einfluss nehmen können oder die sie selbst beeinflussen können (z.B. Erlösung des Patienten von Schmerzen). Diese Beobachtung wurde bereits in publizierten internationalen Studien gemacht. Ein Vergleich zeigt, dass auch hier vielfach als Hauptgründe für die Durchführung von aktiver Sterbehilfe die Vermeidung von "unnüt-

\footnotetext{
${ }^{18}$ Einschränkend muss dieser Studie von Kirschner und Elkeles jedoch hinzugefügt werden, dass hier auch mündliche Interviews geführt wurden.
} 
zem Leiden" und die "Befreiung des Patienten von Schmerzen" (Radulovic und Mojsilovic 1998, S. 413; Verhoef und van der Waal 1997, S. 234) angegeben wurden. Ebenfalls wurde nur von einer Minorität (20\%) das Recht auf einen selbstbestimmten Tod als entscheidendes Kriterium angegeben. Die Teilnehmer der vorliegenden Studie wiesen häufig auf die Missbrauchsgefahr als Argument gegen die Durchführung von aktiver Sterbehilfe hin. In freien Kommentaren wurde hier auf die Gefahr einer "Kosten- Nutzen- Analyse bezüglich des Lebensrechtes von kranken, behinderten und alten Menschen" sowie ein "sozial verträgliche Ableben" aufmerksam gemacht. Dies deutete auf einen emotionalisierten Umgang mit ethisch tiefgehenden Problemen hin. Als weitere wichtige Handlungsmotivation gegen die aktive Sterbehilfe wurde die Möglichkeit einer ärztlichen Fehleinschätzung gesehen und somit erneut ein arztzentrierter Grund benannt. Möglicherweise kann diese Furcht vor Fehleinschätzungen auf weit verbreitete Unsicherheiten im Umgang mit Sterbenden hindeuten, die bei vielen Ärzten offensichtlich vorhanden ist (Schindler et al. 2002). Bei den in dieser Dissertation befragten Allgemeinärzten waren palliative Maßnahmen in der Bewertung der Fallvignetten bei terminal erkrankten Patienten in allen Fällen vorrangig. Als Hinweis hierfür mag das Antwortverhalten in den abgefragten Fallvignetten dienen. Die befragten Ärzte äußerten in allen Fällen die Priorität palliativer Maßnahmen vor der Überlegung zur Durchführung von Sterbehilfe. Diese Ergebnisse stehen im Gegensatz zu denen der 2002 von Schindler et al. veröffentlichten Studie, in der nur 3,5\% der befragten Hausärzte dazu bereit war, eine Zusammenarbeit mit dem palliativmedizinischen Konsiliardienst einzugehen und "häufig" palliativmedizinische Expertisen als "überflüssig" erachteten. Die Autoren hatten festgestellt, dass "sich nur ein kleiner Teil der Hausärzte offensiv mit dem Thema Palliativmedizin" (Schindler et al. 2002, S. 78) auseinander setzte. Diese Aussage konnte anhand der Ergebnisse dieser Studie nicht unterstützt werden: die große Zustimmung palliativer Maßnahmen der befragten Ärzte machte deutlich, dass Palliativmedizin für sie eine wichtige Alternative zur Durchführung von Sterbehilfe darstellt. Die Vernetzung einer suffizienten palliativmedizinischen Ausbildung mit Nachfragen nach Sterbehilfe konnte mehrfach bereits dargestellt werden: Slome et al. konnten in ihrer Untersuchung aus 1997 aufzeigen, dass eine konsequente palliativmedizinische Betreuung in Kombination mit psychotherapeutischer Unterstützung die Suizidwünsche moribunder Patienten signifikant gesenkt hatte (Slome et al. 1997). Auch Quill und Cassel wiesen in der 2003 erschienenen Studie auf das Phänomen hin, dass die verminderte Inanspruchnahme des assistierten Suizids mit einer Verbesserung der hospiz- und palliativmedizinischen Einrichtungen assoziiert gewesen ist (Quill und Cassel 2003).

\subsection{Durchführung von passiver Sterbehilfe}

Bereits drei Viertel $(73,01 \%)$ der befragten Ärzte hatten Nachfragen nach Sterbehilfe erhalten. Im Falle einer Legalisierung erklärten sich knapp sechzig Prozent $(58,82 \%)$ der Ärzte zur Durchführung von Sterbehilfe bereit. Über diesen hohen Grad der Absichtserklärungen hinaus konnte aus der Beantwortung der Fragen geschlossen werden, dass bereits zum jetzigen Zeitpunkt und unter den aktuell gegebenen gesetzlichen Gegebenheiten passive Sterbehilfe zu den durchaus prakti- 
zierten Handlungen deutscher Ärzte gehört. Über die Beantwortung der Frage, ob im Falle der Durchführung von Sterbehilfe Zweifel an der Richtigkeit des eigenen Handelns bestanden hätte, ließ sich indirekt errechnen, dass bereits jeder Dritte der teilnehmenden Allgemeinärzte passive Sterbehilfe durchgeführt hatte. Wie schon bei der aktiven Sterbehilfe wurde als Hauptgrund einer Befürwortung die Erlösung des Patienten von Schmerzen (69,5\%) aufgeführt. Mehrheitlich wurden hier jedoch auch patientenzentrierte Gründe wie das Ermöglichen eines Sterbens in Würde $(65,7 \%)$ oder das Recht auf einen selbstbestimmten Tod $(36,9 \%)$ genannt.

Vergleicht man die Akzeptanz der passiven Sterbehilfe dieser Untersuchung mit der von Kirschner und Elkeles aus 1996 (Kirschner und Elkeles 1998), so ließ sich hier im Gegensatz zur aktiven Sterbehilfe eine breite Zunahme der Zustimmung feststellen. Während 1996 knapp die Hälfte der niedergelassenen Ärzte der indirekten Sterbehilfe zustimmte, waren es in der vorliegenden Studie bereits mehr als drei Viertel.

Diese Entwicklung lässt sich auch international bestätigen: Ward BJ et al. konnten in ihrer Studie aus Großbritannien feststellen, dass die überwiegende Mehrheit (88\%) der befragten Ärzte dazu bereit gewesen wäre, passive Sterbehilfe durchzuführen (Ward BJ und Tate 1994). In einer in 2000 erschienenen Studie von McGlade et al. konnte eine ähnlich hohe Unterstützung (70\%) der passiven Sterbehilfe dargestellt werden; zusätzlich gab die Hälfte der Befragten (49\%) an, bereit zur Durchführung von passiver Sterbehilfe zu sein (Mc Glade et al. 2000). Waddell et al. zeigten in einer Untersuchung aus 1996 an australischen Ärzten (davon 41\% Allgemeinärzte bzw. "General Practitioner") auf, dass der wesentliche Entscheidungsgrund für die Durchführung eines "assisted suicide" der Wunsch des Patienten war (Waddell et al. 1996). Der Vergleich mit internationalen Studien wurde allerdings erschwert, da nur in einigen Fällen speziell auf einzelne Arztgruppen zugegriffen worden ist und insofern ein Vergleich mit der vorliegenden Untersuchung, die sich bewusst auf die Befragung von Allgemeinärzten beschränkte, nur in gewissen Grenzen möglich war. Jedoch machten die Zahlen aus dieser Dissertation tendenziell deutlich, dass Nachfragen nach passiver Sterbehilfe in Deutschland mindestens ebenso häufig wie im Ausland vorkommen und ein Grossteil der befragten Ärzte dem Wunsch nach Sterbehilfe im Falle einer Legalisierung auch nachkommen würde.

Wie auch in dieser Studie wurden in internationalen Publikationen überwiegend patientenzentrierte Gründe als Hauptargument für die Durchführung der (passiven) Sterbehilfe benannt. Dies resultiert aus der breiten gesamtgesellschaftlichen Akzeptanz von passiver Sterbehilfe (Helou et al. 2000), so dass hierbei der strafrechtliche Aspekt weniger relevant ist und der Patient im Mittelpunkt des ärztlichen Handelns stehen kann. 


\subsection{Thematisierung von Sterbehilfe in der medizinischen Ausbildung}

Fast alle der an dieser Untersuchung teilnehmenden Ärzte (88,65\%) fühlten sich während ihrer Ausbildung nicht ausreichend mit den Themen "Sterben" und "Sterbehilfe" konfrontiert. In anderen deutschen Untersuchungen wurde dieser Gegenstand bisher nicht behandelt, und auch ausländische Studien widmen diesem Thema keine besondere Aufmerksamkeit.

Einzig Keane et al. konnten in ihrer 1980 durchgeführten Studie an 950 englischen Allgemeinärzten feststellen, dass die diesbezügliche medizinische Ausbildung unzureichend gewesen ist (Keane et al. 1983). Etwa jeder Zweite (57\%) der Befragten fühlte sich nicht ausreichend in diesem Bereich fortgebildet. Die Studie von Keane et al. machte deutlich, dass auch in anderen Teilen Europas ein Defizit im Umgang mit Sterbenden auszumachen ist. Die Wertung dieses Themas während der ärztlichen Ausbildung wurde von den Universitäten bisher individuell geregelt. In Bonn ist bspw. eine erste Professur für Palliativmedizin eingerichtet worden (Klaschik et al. 2002). Es bleibt zu hoffen, dass diesem Beispiel weitere folgen werden, um dieses Thema bereits Medizinstudenten nahe zu bringen. Durch oft mangelnde Kenntnis im Umgang mit Sterbenden entscheiden Ärzte häufig gegen den Willen des Sterbenden bzw. können diesen nicht adäquat versorgen, falls sie sich diesbezüglich nicht selbst fortgebildet hatten (Gassmann und Schnabel 1996, Oorschot und Schweitzer 2003, Schindler et al. 2002). Daher sollte auch der von Quill und Cassel gemachten Beobachtung einer verminderten Inanspruchnahme von Sterbehilfe bei verbesserter Palliation besondere Aufmerksamkeit geschenkt werden (Quill und Cassel 2003). Es stellt sich daher unbedingt die Forderung nach einer Einbindung dieses Themas in das Studium bzw. einem breiteren Fortbildungsangebot für Mediziner, denn "Ziel sollte es sein Sterbebegleitung als Lernaufgabe aufzufassen, zu diskutieren und zu praktizieren" (Kirschner und Elkeles 1998, S. 249).

\subsection{Der Legalisierungsfaktor}

Sowohl bei der aktiven als auch bei der passiven Sterbehilfe wurde als einer der Hauptgründe für eine Ablehnung die Möglichkeit einer strafrechtlichen Verfolgung genannt (27,47\% bzw. 49,36\%). Es kann daher davon ausgegangen werden, dass der so genannte „Legalisierungsfaktor“ - der Status der Illegalität von Sterbehilfe - bei deutschen Allgemeinärzten eine große Bedeutung besitzt. So würde bei vielen deutschen Ärzten eine Gesetzesänderung das Verhalten gegenüber der Handhabung von Sterbehilfe ändern, was folgende Beispiele verdeutlichen können: Einen Hinweis gaben die Antworten der Fallvignetten (angenommene Legalität der Sterbehilfe), die mit den Antworten im weiteren Fragebogen (tatsächlich gegebene Illegalität) verglichen wurden: Während im Durchschnitt über 90\% der Ärzte passive Sterbehilfe in beiden Fällen durchführen würde, würden von denjenigen, die aktive Sterbehilfe in der Fallvignette durchgeführt hätten, nur die Hälfte bzw. ein Viertel der Befürworter des ersten und dritten Fallbeispiels (Beispiel 1 "Patient leidet an therapieresistenten Schmerzen durch Metastasen" und Beispiel 3 "Patient sorgt sich um seine eigene Versorgungsmöglichkeit") sowie keiner der zweiten und vierten Vignette (Beispiel 2 "Patient sorgt 
sich um die Belastungen, die auf die Familie durch seine Krankheit zukommen" und Beispiel 4 "Patient empfindet sein Dasein als sinnlos") dies ebenso in der momentan juristisch geltenden Situation tun. Es ist daher davon auszugehen, dass eine Legalisierung sich vor allem auf das Verhalten gegenüber der aktiven Sterbehilfe auswirken wird.

Vergleicht man die internationale Haltung von Allgemeinärzten zur Sterbehilfe in Bezug auf den Legalisierungsfaktor, so konnte festgestellt werden, dass sich hier ein sehr inhomogenes Bild darbietet, das stark durch nationale Gesetzgebungen beeinflusst ist: In den Niederlanden sprachen sich bis zu 90\% der befragten Ärzte für (aktive) Sterbehilfe aus. Schon bevor Sterbehilfe legalisiert worden war, gab bereits ein hoher Anteil an, Sterbehilfe zu leisten. Daher kann angenommen werden, dass hier die Legalisierung keinen wesentlichen Einfluss auf die Position zur Sterbehilfe gehabt hat (van der Maas et al. 1991). In Australien stellte sich die Situation ähnlich dar: Auch hier fand Sterbehilfe nicht nur bei der deutlichen Mehrheit von Ärzten Zustimmung, vielmehr gab fast ein Drittel an, Sterbehilfe zu praktizieren (Kuhse und Singer 1988). Dem in Australien vielfach geäußerten Wunsch nach Legalisierung ist bereits regional entsprochen worden.

In Ländern, in denen Sterbehilfe noch nicht legalisiert worden ist, zeigten sich Ärzte vor allem bei der Durchführung von aktiver Sterbehilfe deutlich restriktiver: In Großbritannien fand sich eine große Zustimmung zu Maßnahmen der passiven Sterbehilfe. Aktive Sterbehilfe und Beihilfe zur Selbsttötung wurden hingegen weitgehend abgelehnt (Ward BJ und Tate 1994). Ebenso ließ eine Untersuchung aus dem ehemaligen Jugoslawien eine breite Ablehnung der Sterbehilfe vermuten (Radulovic und Mojsilovic 1998). Die deutlichste Zurückhaltung war aus den skandinavischen Ländern zu vermerken. Zustimmung zur Sterbehilfe konnte man hier bei weniger als ein Drittel der befragten Ärzte finden (Folker et al. 1996; Forde et al. 1997). Vor diesem Hintergrund haben deutsche Ärzte eine Position in der Mitte eingenommen: Während einerseits eine deutliche Mehrheit der Ärzte ihre Bereitschaft zur passiven Sterbehilfe äußerte, und diese auch ohne gesetzliche Regelung praktizierte, hat sich andererseits eine großer Teil gegen die Durchführung von aktiver Sterbehilfe gewendet. Nur knapp ein Viertel der teilnehmenden Ärzte hielt die niederländische Gesetzgebung zur Sterbehilfe für nachahmenswert; hingegen sprach sich mehr als die Hälfte für das bestehende deutsche Gesetz aus.

Sieht man die gefundenen Ergebnisse des Einflusses des "Legalisierungsfaktors" im Vergleich zu den Positionen der deutschen Ärzteschaft, die in den Grundsätzen der Bundesärztekammer zur Sterbebegleitung 1998 verfasst worden sind (Bundesärztekammer 1998), so stellte man fest, dass diese theoretische Position sich grundsätzlich von der praktischen Handhabung der Ärzte unterschieden hat. Der Arzt steht vor dem Problem des "Handelns unter Unsicherheit" (Wehkamp 1997, S. 32), da in Deutschland im Vergleich zum Ausland wenig öffentlicher Diskurs zum Thema herrscht und kaum wissenschaftliche Studien zum Thema existieren. Somit werden juristische 
Vorgaben oft zum Hauptelement des ärztlichen Handelns, obwohl diese ebenfalls meist unzureichend sind (Taupitz 2001). Strafrechtler wiesen darauf hin, dass sich der Gesetzgeber seiner "Regelungsverantwortung bezüglich passiver und indirekter Sterbehilfe, Patientenverfügungen und Vorsorgevollmacheten" entzogen hat (Gahl 2002, S. 867). Ferner mahnten sie an, dass bei einer isolierten Betrachtung der juristischen Vorgaben durch den Arzt der Blick für das Humane verloren gehen könne. Sie schätzten das Recht in Würde zu sterben gewichtiger ein, als bspw. ein Recht auf aktive Sterbehilfe und appellieren daher als ersten Schritt an die Ausschöpfung aller palliativmedizinischer Mittel durch den Arzt (Niethammer 1995). In den Grundsätzen der Bundesärztekammer zur Sterbebegleitung ist sowohl die aktive als auch "eine gezielte Lebensverkürzung durch Maßnahmen, die den Tod herbeiführen oder das Streben beschleunigen sollen" (Bundesärztekammer 1998, A-2366) unzulässig und mit Strafe bedroht. Es müsste nach den vorliegenden Ergebnissen nun überlegt werden, ob und in wie weit der momentan praktizierte Umgang mit der Sterbehilfe in einer überarbeiteten Ausgabe dieser Grundsätze festgehalten werden kann. Bereits 1986 wurde von Strafrechtlern und Medizinern ein "fakultatives Absehen von Strafe" (Gahl 2002, S. 868) bei der Durchführung von Sterbehilfe angeregt. Dieser Vorschlag fand allerdings keinen Konsens.

\subsection{Beziehung zwischen Euthanasie im sog. "Dritten Reich" und der ärztlicher Ster- behilfe}

Die überwiegende Mehrheit der teilnehmenden Ärzte $(79,13 \%)$ sah keinen Zusammenhang zwischen Euthanasie im sog. "Dritten Reich" und der ärztlichen Sterbehilfe. Von den Ärzten, die eine solche Verbindung gezogen haben, würden unter der jetzigen Gesetzeslage ein Drittel passive und sogar sechs Prozent aktive Sterbehilfe durchführen. Im Falle einer Legalisierung wären doppelt so viele - also mehr als jeder Zehnte - dazu bereit, aktive Sterbehilfe durchzuführen. Dies stellt mögliche Konsequenzen einer Legalisierung von Sterbehilfe dar, nämlich eine breitere Inanspruchnahme der (aktiven) Sterbehilfe. Erstmals konnte durch diese Frage analysiert werden, ob die spezifisch deutsche Vergangenheit Einfluss auf aktuelle Sterbehilfepraktiken hat. Zwar wurde in bereits publizierten Arbeiten auf dieses Phänomen hingewiesen, ohne dass jedoch ein Zusammenhang untersucht worden war (Müller- Busch et al. 2003). Tatsächlich zeigten überraschenderweise die Ergebnisse der vorliegenden Untersuchung, dass deutsche Allgemeinärzte ihre Position zur Sterbehilfe weitgehend unbeeinflusst von der jüngeren deutschen Geschichte und den Verbrechen im "Dritten Reich" darstellten. Allerdings muss an dieser Stelle erwähnt werden, dass ein genereller Einfluss der spezifischen deutschen Vergangenheit auf ärztliche Positionen dennoch nicht ganz ausgeschlossen werden kann, auch wenn er von den Befragten negiert wird. Es kann angenommen werden, dass schon durch den gesamtgesellschaftlichen Diskurs, der seit Beginn der 80er Jahre die medizinischen Verbrechen im Nationalsozialismus in das Bewusstsein brachte (Baader und Schultz 1980), auch medizinethische Positionen zumindest beeinflusst wurden und werden. Ein dergestalt "unterschwellig" anzunehmender Einfluss konnte allerdings im Rahmen dieser 
durchgeführten Untersuchung anhand einiger weniger dichotomer Fragen aus verständlichen Gründen nicht erarbeitet werden. Es wäre wünschenswert, dass sich nachfolgende Studien noch weiter mit der Beziehung des Nationalsozialismus auf die heutige Sterbehilfedebatte beschäftigen, um hier weitere Einflussfaktoren aufzeigen zu können.

\subsection{Einflussfaktoren bezüglich Einstellungen zur Sterbehilfe}

Es existierten große Divergenzen bezüglich der Einstellung zur Sterbehilfe, die in Abhängigkeit vom Zeitpunkt der Approbation, einem erlebten Tätigkeitsbereich, der Konfession sowie dem Ort der Praxis gesehen werden konnten. So hatte sich in dieser Studie der überwiegende Teil der Befürworter von Sterbehilfe (71,96\%) in den Jahren 1965-1983 Jahren approbiert. Bei der jüngeren Generation hingegen, die nach 1983 approbiert wurde, ließ sich wieder eine gegenläufige Tendenz und somit ablehnende Haltung zur Sterbehilfe feststellen (36,51\%).

In der 1996 erschienenen australischen Studie von Waddell et al. (Waddell et al. 1996) ließen sich diese Differenzen im Verhalten gegenüber ärztlich assistiertem Tod nicht feststellen. So gaben die seit mehr als 21 Jahren qualifizierten Ärzte ähnlich häufig wie ihre jüngeren Kollegen an, aktive Sterbehilfe leisten zu wollen (4,7\% vs. 5,4\%). Auch hier wurde der Vergleich mit den Ergebnissen der vorliegenden Dissertation durch die Vermengung verschiedener Fachrichtungen erschwert. Eine mögliche Erklärung für die gefundenen Ergebnisse dieser Studie wäre, dass die jüngere Generation durch die zunehmende Aufklärung und Bearbeitung des Nationalsozialismus im Bezug zur Sterbehilfe geprägt und sensibilisiert worden ist, so dass diese Generation der Sterbehilfe eher negativ gegenüber stand.

Ärzte, die sich in ihrer Ausbildung intensiver mit den Themen Tod und Sterben auseinander setzten mussten (z. B. in den Fachgebieten Onkologie, Geriatrie) und hiermit auch häufiger konfrontiert sind, befürworteten aktive und passive Sterbehilfe signifikant häufiger als Kollegen, die dies nicht tun (z. B. Psychiater). Dies lässt darauf schließen, dass eine Schulung des Themas bzw. eine häufige Konfrontation mit Sterbenden die Angst vor der Durchführung von Sterbehilfe nehmen könnte. Weitere Studien werden benötigt, um dies weiter untersuchen zu können.

Ferner waren vor allem (12,84\%) Ärzte aus kleineren Städten (bis 50.000 Einwohner) dazu bereit, aktive Sterbehilfe durchzuführen. Dies könnte sich auf die sozial engere Betreuung von Patienten in Kleinstädten (längere Behandlungsdauer, weniger Arztwechsel) und deren bessere Kenntnis zurückführen lassen. Hierdurch wurde möglicherweise eher einem Wunsch nach aktiver Sterbehilfe entsprochen. Ferner könnte angenommen werden, dass in kleineren Orten (sozial) übergeordnete Kontrollinstanzen eher fehlten als in Grosstädten. Weiterhin kann diskutiert werde, dass in kleineren Orten die sozialen Pflichten eines Arztes größer als vergleichsweise in größeren Städten waren, die eine gewisse Anonymität garantierten. Die Bereitschaft, passive Sterbehilfe zu leisten, war in kleinen Orten ähnlich hoch wie in größeren Städten ab 51.000 Einwohnern (84,73\% vs. 
75,83\%). Dies lässt sich erneut durch die gesamtgesellschaftlich größere Akzeptanz der passiven Sterbehilfe erklären.

Verglichen mit den Daten von Lee et al. aus der 1996 erschienen Studie aus Oregon (Lee et al. 1996), waren hier jedoch andere Ergebnisse darstellbar: Jeder Zweite (50\%) aus größeren (ab 250.000 Einwohner) und mittleren (25.000-250.000 Einwohner) Städten war bereit, den ärztlich assistierten Selbstmord zu begleiten, in den kleineren Städten (weniger als 25.000 Einwohner) hingegen nur etwa jeder Dritte (38\%). Diese unterschiedlichen Ergebnisse jedoch müssen differenziert betrachtet werden: Durch die verschiedenen Erhebungsmethoden und unterschiedliches Teilnehmerkollektiv wurde auch hier der Vergleich der Studien erschwert.

Es konnte in der vorliegenden Untersuchung gezeigt werden, dass signifikant häufiger diejenigen Ärzte aktiver und passiver Sterbehilfe zustimmten (72,97\% vs. 76,67\%), die bereits Nachfragen hiernach erhalten hatten. Dieser Einflussfaktor wurde bisher in keiner anderen Studie festgestellt. Es bleibt daher die Frage nach möglichen weiteren Einflussfaktoren zur Durchführung von Sterbehilfe zu beantworten. Diskutiert werden könnte hierbei über eine mögliche Fortbildung der angesprochenen Ärzte zum Thema oder aber einen durch die Nachfragen unterschwellig ausgeübten Druck seitens der Patienten. Daher sollte eine differenziertere Betrachtung dieses Einflussfaktors in noch folgenden Studien durchgeführt werden.

Weiterhin wurde in der vorliegenden Studie dargestellt, dass tief religiöse Menschen - vor allem Katholiken- gegen jede Form von Sterbehilfe sind (47,37\%). Diese Ergebnisse waren kongruent zu anderen deutschen und internationalen Studien (Kirschner und Elkeles 1998; Lichtenstein 1997; Singh 1979; Ward RA 1980). Gründe für diese breite Ablehnung der Sterbehilfe sind noch nicht untersucht worden; vorstellbar wäre eine andere Bewertung göttlich gegebenen Lebens.

\subsection{Vergleich mit den Niederlanden bei der Nachfrage nach Sterbehilfe}

Über die Hälfte $(51,45 \%)$ der befragten Ärzte gab an, in den letzten zehn Jahren etwa zwei bis fünf mal um aktive Sterbehilfe und mehr als fünfmal um passive Sterbehilfe $(50,93 \%)$ gebeten worden zu sein. Anhand dieser Zahlen konnte insofern geschätzt werden, dass deutsche Patienten im Schnitt etwa 0,2-0,5-mal im Jahr um aktive und mehr als 0,5-mal pro Jahr nach passiver Sterbehilfe gebeten hatten. Damit war die Häufigkeit von Nachfragen nach Sterbehilfe in dieser Studie mit holländischen Angaben aus dem Jahre 1991 - also vor der Legalisierung von Sterbehilfe - (0,250,8 Nachfragen/Jahr) vergleichbar (van der Maas et al. 1991). Wie auch in einer weiteren niederländischen Studie aus den Jahren 1986-1989, hatten auch in Deutschland rund drei Viertel der Ärzte $(73,01 \%$ vs. $73 \%)$ bereits Nachfragen nach Sterbehilfe erhalten (Verhoef und van der Wal 1997). Zu dem Zeitpunkt dieser Befragung war in Holland die Sterbehilfe zwar noch nicht legalisiert, die Diskussion wurde allerdings schon viel breiter und offener geführt als derzeit noch in 
Deutschland. Dass sich trotz eines fehlenden breiten öffentlichen Diskurses oder einer entsprechenden Legalisierung die Nachfragen von Patientenseite nach Sterbehilfe ähnelten, könnte aufzeigen, dass die Menschen am Ende ihres Lebens und Leidens autonom über ihren Körper entscheiden und sich in dieser persönlichen Entscheidung nicht durch nationale Gesetzgebungen beeinflussen lassen wollten. Um hier jedoch genauere Aussagen treffen zu können, sind weitere Untersuchungen erforderlich. Wie der Vergleich der Nachfragen nach Sterbehilfe mit den Niederlanden ebenfalls zeigen konnte, setzten sich deutsche Patienten offensichtlich im privaten Kreis stärker mit der Sterbehilfethematik auseinander, als es die relativ geringe öffentliche Debatte vermuten lassen würde.

\subsection{Einstellung zur Sterbehilfe im internationalen Vergleich}

Die überwiegende Mehrheit der befragten deutschen Allgemeinärzte unterstützte die passive Sterbehilfe und hielt sie für ein akzeptables Vorgehen (80,35\%). Hingegen fand sich eine breite Ablehnung in Bezug auf die aktive Sterbehilfe (65,28\% hielten aktive Sterbehilfe für kein akzeptables Vorgehen). Weiterhin konnte die vorliegende Untersuchung deutlich machen, dass sich deutsche Allgemeinärzte vergleichsweise restriktiv bei der Durchführung der aktiven Sterbehilfe verhielten. Gab in der vorliegenden Studie knapp jeder Fünfte $(18,27 \%)$ an, bei Legalisierung aktive Sterbehilfe durchzuführen, so waren dies in amerikanischen und australischen Studien deutlich mehr. In einer Studie aus Washington von 1994 gab bereits mehr als jeder Dritte (33\%) an, dass es Situationen gegeben hätte, in der er an der Durchführung von Euthanasie teilgenommen hätte (Cohen et al. 1994). In einer Studie aus New South Wales wurden ebenfalls Ärzte verschiedener Fachrichtung zum Thema Sterbehilfe befragt, was erneut die Vergleichbarkeit der Studien erschwerte. Trotzdem sollte nicht unerwähnt bleiben, dass in dieser Studie jeder Zweite (50\%) die Bitte um Sterbehilfe unter der Prämisse der Legalität von aktiver Sterbehilfe eines unheilbaren Patienten erfüllen würde (Baume und O' Malley 1994). Im Gegensatz zu diesen Ergebnissen, die sich jedoch deutlich von den Resultaten dieser Studie unterschieden, zeigten sich jedoch bei der Durchführung der Sterbehilfe Gemeinsamkeiten zwischen deutschen Allgemeinärzten und ihren irischen Kollegen. Letztere hielten mit einer Mehrheit von $70 \%$ die Durchführung von passiver Sterbehilfe für moralisch und ethisch richtig, nur knapp jeder Fünfte (23\%) gab dies für die aktive Sterbehilfe an (Mc Glade et al. 2000). Dass also sowohl Patienten - wie es der Vergleich mit den Niederlanden deutlich machte - als auch Ärzte - wie es der Vergleich mit Irland aufzeigte - ähnliche Einstellungen bezüglich der (passiven) Sterbehilfe wie ihre internationalen Mitbürger hatten, könnte auf die geringe Bedeutung eines öffentlichen Diskurses zur Entscheidungsfindung in der Bevölkerung hindeuten.

\subsection{Schlussfolgerungen}

In Deutschland besteht ein großer Diskussionsbedarf zum Thema Sterbehilfe. 
Das breite Interesse der befragten Ärzte an den Ergebnissen dieser Studie und die beträchtliche Anzahl an angefügten Kommentaren ließen darauf schließen, dass es für deutsche Allgemeinärzte noch immer zu wenig Möglichkeiten gibt, sich ausreichend mit diesem Thema zu beschäftigen, obwohl Sterbehilfe zu einem zentralen Aspekt ihrer Arbeit gehört. Die hieraus resultierenden Unsicherheiten schlugen sich nicht nur in der Schwierigkeit der Begriffsabgrenzung nieder, sondern auch im rechtlichen Umgang mit der Durchführung von Sterbehilfe. Für den Grossteil der befragten Ärzte waren juristische Bedenken der Hauptgrund gegen vor allem aktive Sterbehilfe. Obwohl Sterbehilfe in den meisten anderen Gebieten wie den Northern Territories, Oregon, in Irland oder früher in den Niederlanden illegal war, wurden hier weniger strafrechtliche, sondern primär persönliche und patientenzentrierte Gründe zur Entscheidungsfindung angegeben (Baume und O' Malley 1994; Dickinson et al. 1996; Fried et al 1993; van der Waal et al. 1992). Diese Gründe wurden in der vorliegenden Studie tendenziell stärker bei der Durchführung von passiver Sterbehilfe benannt, was auf eine verstärkte Arzt-Patienten Interaktion bei der Durchführung der passiven Sterbehilfe hindeutete. Ferner herrschte eine allgemein größere Akzeptanz bei der Durchführung von passiver Sterbehilfe, weshalb der strafrechtliche Aspekt hier weniger zum Tragen gekommen ist. Weiterhin konnte diese Studie zeigen, dass der oft beschworene "deutsche Sonderweg" zumindest praktisch in der ärztlichen Praxis keinen Niederschlag gefunden hatte. Weder sahen die befragten Ärzte eine Beziehung zwischen Euthanasie im Dritten Reich und der heutigen ärztlichen Sterbehilfe, noch ließen sich gravierende Unterschiede in ihren Handlungsweisen und Einstellungen zur Sterbehilfe im Vergleich zu ihren ausländischen Kollegen feststellen. Dennoch zeigte sich eine im internationalen Vergleich verstärkte Ablehnung gerade aktiver Sterbehilfe, so dass angenommen werden konnte, dass noch weitere Faktoren auf die Haltung deutscher Allgemeinärzte Einfluss nahmen. Als mögliche Erklärung war eine verstärkte gesamtgesellschaftliche Diskussion der medizinischen Verbrechen im Nationalsozialismus denkbar, die ethisch prägend quasi als "präsenter Hintergrund" vorhanden gewesen ist, ohne dass die Ärzte sich in ihrer einzelnen Entscheidung hierauf beriefen. Es stellt sich diesbezüglich die Forderung nach klinischen Richtlinien wie sie bspw. in den USA bereits eingeführt worden sind (Miller et al. 1994). Diese Richtlinien wurden enger als bspw. die der Niederlanden gefasst und sollten dadurch frühzeitig Missbrauchstendenzen erkennen und somit verhindern. Weiterhin ist zu fragen, inwieweit durch die vorliegende Untersuchung die bestehenden Grundsätze zur ärztlichen Sterbebegleitung in Frage gestellt werden oder sich als realitätsfern erweisen: Die vorliegende Untersuchung hat belegt, dass Nachfragen - auch nach aktiver Sterbehilfe - keine Seltenheit in der ärztlichen Praxis gewesen sind. Daher sollte auch die Bundesärztekammer mit ihren Bemühungen fortfahren, die Qualität von Patientenverfügungen zu sichern und eigene Empfehlungen hierzu auszusprechen, wie sie es in einem bereits erschienenen Ratgeber erstmals tat (Bundesärztekammer 1999). Werden solche Verfügungen von Arzt und Patient gemeinsam erarbeitet, kann ein Austausch über Todes- und Sterbevorstellungen stattfinden, durch die der Arzt sich von der Einwilligungsfähigkeit des Patienten überzeugen kann. Es hatte sich außerdem gezeigt, dass die Konfrontation und das Auseinandersetzen mit dem Thema Sterben von 
ganz elementarer Bedeutung für die Ausbildung von Studenten und jungen Ärzten sein sollte, da hier offensichtliche Defizite auszumachen gewesen sind. Kaum ein Arzt in dieser Studie hatte sich während seiner Ausbildung mit diesem Thema auseinandergesetzt. Erst während seiner praktischen Berufsausübung ist der Großteil damit konfrontiert worden. Eine schlechte palliative oder medizinethische Ausbildung korrelierte mit einer überwiegend ablehnenden Haltung jeder Form der Sterbehilfe.

In einer zunehmend alternden Gesellschaft wird das Thema Sterbehilfe zunehmend an Brisanz gewinnen und verstärkt als elementarer Bereich allgemein- und hausärztlicher Tätigkeit angesehen werden. Fragen zur Sterbehilfe stellen somit "aktuell eine ganz zentrale Herausforderung an die Allgemeinmedizin und werden dies auch in Zukunft" tun (Brody 1993, S. 125). 


\section{Zusammenfassung}

Zielsetzung: Empirische Erhebung von Beurteilungen und Einstellungen deutscher Allgemeinärzte zur Sterbehilfe.

Design: Deutschlandweite anonyme postalische Fragebogenerhebung

Methoden: 500 Allgemeinärzte aus ganz Deutschland wurden nach Randomisation aus dem Telefonverzeichnis der deutschen Telekom ausgewählt. Diesen wurde per Post ein vorher bereits pilotierter Fragebogen mit einem bereits frankierten Rückumschlag zugesendet. In dem Fragebogen wurden die Teilnehmer über Entscheidungen todkranker Patienten in hypothetischen Fallvignetten befragt. Außerdem sollten sie über Einstellungen und Handlungsweisen gegenüber Sterbehilfe Auskunft geben. Einen Monat nach dem Versenden der Fragebögen wurde ein Erinnerungsschreiben an alle Teilnehmer geschickt.

Ergebnisse: Der Fragebogen wurde von 233 der 481 erreichbaren Allgemeinärzte zurückgesendet, was einer Antwortrate von 48\% entspricht. Obwohl die Möglichkeit, Sterbehilfe durchzuführen in den Fallvignetten nur sehr selten gewählt wurde, hielten 35\% bzw. 80\% der Teilnehmer die aktive bzw. passive Sterbehilfe für ein akzeptables Vorgehen. Als Hauptargument für die Durchführung von Sterbehilfe wurden nicht beherrschbare Schmerzen und das Ermöglichen eines würdevollen Sterbens genannt - hingegen wurde die Möglichkeit eines Missbrauches von Sterbehilfe und einer ärztlichen Fehleinschätzung als Hauptgrund dagegen benannt. 79\% der antwortenden Ärzte hielten einen Vergleich zwischen der Euthanasie im sog. "Dritten Reich" und der Durchführung von Sterbehilfe heutzutage für nicht gerechtfertigt. $62 \%$ hatten bereits Nachfragen nach aktiver und 73\% nach passiver Sterbehilfe erhalten. 13\% der Teilnehmer gaben an, bereits selbst aktive Sterbehilfe durchgeführt zu haben, 38\% hatten passive Sterbehilfe durchgeführt. Im Falle einer Legalisierung von Sterbehilfe gaben $18 \%$ bzw. 59\% an, aktive bzw. passive Sterbehilfe durchführen zu wollen.

Schlussfolgerungen: Die meisten deutschen Allgemeinärzte befürworteten die Durchführung palliativmedizinischer Maßnahmen bei todkranken Patienten. Nichtsdestotrotz waren bei ihnen Nachfragen und die Durchführung von Sterbehilfe nicht selten. Der Großteil der befragten Ärzte hielt eine gesetzliche Regelung zur Sterbehilfe als Entscheidungshilfe am Lebensende für erforderlich. Die spezielle deutsche Geschichte stellte keinen entscheidenden Einflussfaktor bei Einstellungen und Verhaltensweisen zur Sterbehilfe dar. 
8. Anhang

8.1 Fragebogen 
Abteilung Allgemeinmedizin - Universität Göttingen

\section{Allgemeinärztliche Einstellungen zur Sterbehilfe}

Teil 1: Fallbeispiele

\section{Anmerkung:}

Bitte beantworten Sie die folgenden Fallbeispiele unter der Annahme, dass sowohl

aktive als auch passive Sterbehilfe in Deutschland legal seien.

1. Ein voll zurechnungsfähiger Patient leidet an einem fortgeschrittenem Prostata-Karzinom mit Knochenmetastasen und hat voraussichtlich nur noch wenige Monate zu leben. Es besteht keine Aussicht auf eine Heilung oder eine Verminderung des Krankheitsprogresses. Trotz Ausschöpfung aller palliativen Maßnahmen wie NSAID, Morphin, Hormontherapie, Strahlentherapie, Nervenblockaden etc. verursachen die Metastasen unerträgliche Schmerzen. Sie betreuen den Patienten seit zwei Jahren. Er wurde von einem Psychiater als nicht depressiv beurteilt und bittet Sie nun zum wiederholten Male um Sterbehilfe.

Wie würden Sie handeln?

$\square \quad$ Ich lehne die Durchführung von Sterbehilfe ab und versorge den Patienten mit palliativen Maßnahmen so gut wie möglich.

$\square \quad$ Ich verordne dem Patienten eine große Menge an Morphin und instruiere inn, in welcher Weise die Einnahme zum Tod führt.

$\square \quad$ Ich verzichte auf die weitere Behandlung des Patienten

$\square \quad$ Ich verabreiche dem Patienten eine Injektion mit einer tödlichen Menge eines Medikamentes

$\square \quad$ Keine der angegebenen Möglichkeiten, sondern

2. Ein voll zurechnungsfähiger Patient leidet unter einem fortgeschrittenen Malignom und hat voraussichtlich nur noch wenige Monate zu leben. Es bestehen keine Aussichten auf eine Heilung oder eine Verminderung des Krankheitsprogresses. Unter der jetzigen Therapie ist der Patient schmerzfrei und kann für sich selbst sorgen. Er ist jedoch zunehmend besorgt über die Belastung, die durch die Verschlechterung der Krankheit und den Tod auf seine Familie zukommen wird. Der Patient wurde von einem Psychiater als klinisch nicht depressiv beurteilt, fragt aber zum wiederholten Male nach einer lebensbeendenden Injektion.

Wie würden Sie handeln?

Ich lehne die Durchführung von Sterbehilfe ab und versorge den Patienten mit palliativen Maßnahmen so gut wie möglich.

$\square \quad$ Ich verordne dem Patienten eine große Menge an Morphin und instruiere inn, in welcher Weise die Einnahme zum Tod führt.

$\square \quad$ Ich verzichte auf die weitere Behandlung des Patienten

$\square \quad$ Ich verabreiche dem Patienten eine Injektion mit einer tödlichen Menge eines Medikamentes

$\square \quad$ Keine der angegebenen Möglichkeiten, sondern

3. Ein voll zurechnungsfähiger Patient leidet unter einem fortgeschrittenen Malignom und hat voraussichtlich nur noch wenige Monate zu leben. Es bestehen keine Aussichten auf eine Heilung oder eine Verminderung des Krankheitsprogresses. Unter der jetzigen Therapie ist der Patient schmerzfrei. Er kann jedoch weder das Bett verlassen noch sich selbst versorgen. Der Patient wurde von einem Psychiater als klinisch nicht depressiv beurteilt, fragt aber zum wiederholten Male nach einer lebensbeendenden Injektion.

Wie würden Sie handeln?

Ich lehne die Durchführung von Sterbehilfe ab und versorge den Patienten mit palliativen Maßnahmen so gut wie möglich.

$\square \quad$ Ich verordne dem Patienten eine große Menge an Morphin und instruiere ihn, in welcher Weise die Einnahme zum Tod führt.

$\square \quad$ Ich verzichte auf die weitere Behandlung des Patienten

$\square \quad$ Ich verabreiche dem Patienten eine Injektion mit einer tödlichen Menge eines Medikamentes

$\square \quad$ Keine der angegebenen Möglichkeiten, sondern

4. Ein voll zurechnungsfähiger Patient leidet unter einem fortgeschrittenen Malignom und hat voraussichtlich nur noch wenige Monate zu leben. Es bestehen keine Aussichten auf eine Heilung oder eine Verminderung des Krankheitsprogresses. Unter der jetzigen Therapie ist der Patient schmerzfrei und kann sich selbst versorgen, findet sein Leben aber sinn- und zwecklos. Der Patient wurde von einem Psychiater als klinisch nicht depressiv beurteilt, fragt aber zum wiederholten Male nach einer lebensbeendenden Injektion.

Wie würden Sie handeln?

Ich lehne die Durchführung von Sterbehilfe ab und versorge den Patienten mit palliativen Maßnahmen so gut wie möglich. Ich verordne dem Patienten eine große Menge an Morphin und instruiere inn, in welcher Weise die Einnahme zum Tod führt. Ich verzichte auf die weitere Behandlung des Patienten

Ich verabreiche dem Patienten eine Injektion mit einer tödlichen Menge eines Medikamentes

Keine der angegebenen Möglichkeiten, sondern 


\section{Teil 2}

1. Haben Sie selbst oder ein Angehöriger unter einer lebensbedrohlichen Erkrankung gelitten ?

$\square$ ja

$\square \quad$ nein

2. Haben Sie von Patientenseite schon Nachfragen nach Sterbehilfe erhalten ?
Aktive Sterbehilfe
Passive Sterbehilfe
$\square$ ja
$\square \quad$ ja
nein
$\square \quad$ nein

Wenn ja, wie oft innerhalb der letzten 10 Jahre?
Aktive Sterbehilfe
Passive Sterbehilfe
$\square \quad$ Einmal
$\square \quad$ Einmal
$\square \quad 2-5 \mathrm{mal}$
$\square \quad 2-5$ mal
$\square>5 \mathrm{mal}$
$\square>5 \mathrm{mal}$

3. Mit welcher der folgenden Vorgehensweisen könnten Sie sich identifizieren, wenn Sie von Patienten um Sterbehilfe gebeten werden würden? (Mehrere Antworten möglich)

$\square \quad$ Ich lehne die Durchführung von Sterbehilfe ab

$\square \quad$ Ich führe aktive Sterbehilfe durch

$\square \quad$ Ich führe passive Sterbehilfe durch

$\square \quad$ Ich zeige dem Patienten mögliche Alternativen auf (z.B. palliative Maßnahmen)

$\square \quad$ Ich lasse den Patienten psychiatrisch untersuchen

$\square \quad$ Ich berate mich mit

$\begin{array}{ll}\square & \text { Kollegen } \\ \square & \text { Pflegepersonal } \\ \square & \text { Angehörigen des Patienten } \\ \square & \text { Geistlichen } \\ \square & \text { anderen, }\end{array}$

4. Falls Sie jemals Sterbehilfe durchgeführt haben, glauben Sie auch rückblickend, das Richtige getan zu haben?
Aktive Sterbehilfe
Passive Sterbehilfe
$\square$ ja
$\square \quad$ ja
$\square \quad$ nein
$\square \quad$ nein
$\square \quad$ unsicher, weil
$\square$ unsicher, weil

5. Halten Sie Sterbehilfe durch Ärzte für ein akzeptables Vorgehen?

\section{Aktive Sterbehilfe}

$\square \quad$ ja, unter bestimmten Umständen nein, unter keinen Umständen

\section{Passive Sterbehilfe}

$\square \quad$ ja, unter bestimmten Umständen

$\square \quad$ nein, unter keinen Umständen

Welche der folgenden Kriterien spielen für diese Entscheidung eine Rolle ? (Mehrere Anworten möglich)

Erlösung des Patienten von Schmerzen

Recht auf einen selbstbestimmten Tod

Ermöglichen eines Sterbens in Würde

Befreiung der Angehörigen von Lasten

Entlastung des Gesundheitssystems

Religiöse Gründe

Eid des Hippokrates

Historische Gründe ("Drittes Reich")

Juristische Gründe

Ausreichende Palliativmedizin

Mögliche ärztliche Fehleinschätzung

Möglichkeit des Missbrauchs

Andere:

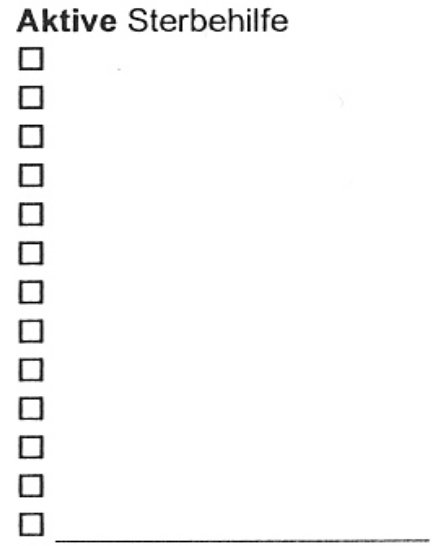

Passive Sterbehilfe

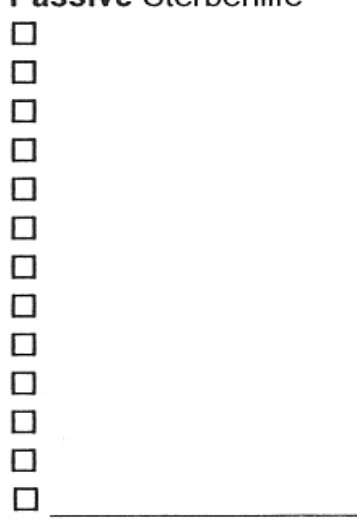


6. Sofern Sie die Durchführung von Sterbehilfe nicht generell ablehnen: Welche Bedingungen könnten eine solche Entscheidung für Sie erleichtern? (Mehrere Antworten möglich)

Keine strafrechtliche Verfolgung

Unabhängige Beurteilung durch andere Ärzte

Wiederholter Wunsch des Patienten

Schriftlicher Wunsch des Patienten

Zustimmung der Angehörigen

Ablehnung palliativer Massnahmen durch den Patienten

Ausschluss einer psychischen Erkrankung

Volle Zurechnungsfähigkeit

Begrenzte Lebenserwartung (z.B.< 6 Mon.)

Nicht beherrschbare Schmerzen

Gute Kenntnis / Lange Betreuung des Patienten

Mindestalter des Patienten

Andere:

\begin{tabular}{l} 
Aktive Sterbehilfe \\
$\square$ \\
$\square$ \\
$\square$ \\
$\square$ \\
$\square$ \\
$\square$ \\
$\square$ \\
$\square$ \\
$\square$ \\
$\square$ \\
$\square$ \\
$\square$ \\
$\square$ \\
\hline
\end{tabular}

Passive Sterbehilfe

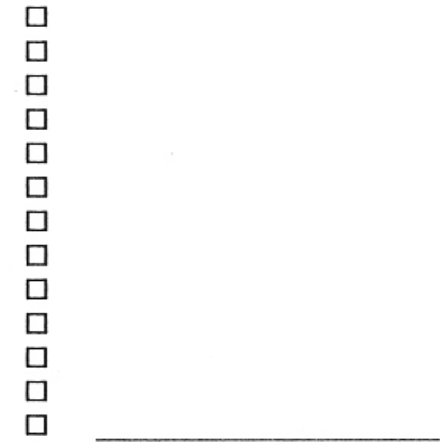

7. Besteht für Sie ein moralisch relevanter Unterschied zwischen der Durchführung aktiver Sterbehilfe

(z.B. der Injektion eines tödlichen Medikaments) und passiver Sterbehilfe (z.B. Zurverfügungstellung und Instruktion der Einnahme einer tödlichen Dosis eines Medikaments)?

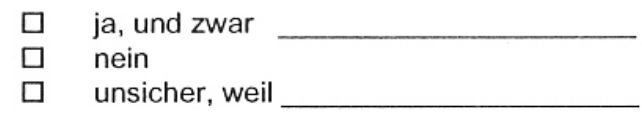

8. Haben Sie jemals Mittel zur Durchführung eines Suizids zur Verfügung gestellt?
$\square$ ja
$\square \quad$ nein
$\square$ unsicher, weil

9. Halten Sie in Deutschland eine gesetzliche Regelung zur Sterbehilfe für erforderlich?
Aktive Sterbehilfe
Passive Sterbehilfe
ja
$\square \quad$ nein
$\square \quad$ ja
$\square$ unsicher, weil
$\square \quad$ nein

10. Wären Sie bereit, Sterbehilfe durchzuführen, wenn dafür in Deutschland eine gesetzliche Grundlage bestünde ?
Aktive Sterbehilfe
Passive Sterbehilfe
$\square \quad$ ja
$\square \quad$ nein
unsicher, weil

$\begin{array}{ll}\square & \text { ja } \\ \square & \text { nein } \\ \square & \text { unsicher, weil }\end{array}$

11. Es existieren unterschiedliche Modelle zur Sterbehilfe. Welches der folgenden Modelle würden Sie favorisieren ?

$\square \quad$ In den Niederlanden wurde die aktive Sterbehilfe legalisiert. Um als Arzt Sterbehilfe durchzuführen, müssen bestimmte Voraussetzungen erfüllt sein. Die wichtigsten Punkte lauten: Der Patient muss mehrfach um Sterbehilfe gebeten haben, an einer tödlichen Krankheit mit infauster Prognose leiden und über Alternativen wie Palliativmedizin informiert worden sein. Schließlich muss vor der Tötung ein weiterer Arzt hinzugezogen werden. Die Entscheidung des Arztes, Sterbehilfe durchzuführen, wird von einem unabhängigen Gremium überprüft.

$\square \quad$ In der Schweiz besteht für voll zurechnungsfähige Personen die Möglichkeit, als Mitglied einer Organisation Hilfe bei der Sterbevorbereitung, der Sterbegleitung sowie der Durchführung von Patientenverfügungen gegenüber Ärzten, Kliniken, Alters- und Pflegeheimen zu erhalten. Gegen einen Mitgliedsbeitrag hilft diese Organisation durch“ personelle, finanzielle und juristische Möglichkeiten” bei "ärztlich diagnostizierten hoffnungslosen oder unheilbaren Krankheiten, unerträglichen Schmerzen oder unzumutbare Behinderungen“.

$\square \quad$ In Deutschland ist die aktive Sterbehilfe sowie das Töten auf Verlangen strafbar. Die Nichtaufnahme oder Beendigung einer Intensivbehandlung ist nur dann nicht strafbar, wenn sie aus einer frei verantwortlichen Willensentscheidung des Patienten (z.B. Patientenverfügung) entstanden ist. Unter bestimmten Voraussetzungen ist der Abbruch lebenserhaltender Massnahmen zulässig, wenn dies dem "mutmasslichen" Willen des Patienten entspricht.

12. Fühlten Sie sich während Ihrer Ausbildung zum Arzt ausreichend mit den Themen Sterben und Sterbehilfe konfrontiert ?

口 ja

$\square \quad$ nein

13. Halten Sie einen Vergleich zwischen der Euthanasie im sog. "Dritten Reich" und der Durchführung von Sterbehilfe heutzutage für gerechtfertigt?

$\square \quad$ ja, weil

$\square \quad$ nein, weil

Bitte wenden 


\section{Teil 3: Persönliche Angaben}

1. Alter

2. Geschlecht

3. Familienstand

4. Kinder

5. Religion

Religionsausübung
Jahre

$\square \quad$ weiblich

$\square \quad$ männlich

$\square \quad$ ledig

$\square$ verheiratet /eheähnl. Gemeinschaft

$\square$ feste Partnerschaft

$\square$ geschieden / getrennt lebend

$\square$ verwitwet

$\square \quad$ ja

$\square \quad$ nein
$\square$ evangelisch
katholisch
$\square$ andere,
$\square \quad$ keine

$\square \quad$ aktiv

$\square$ gelegentlich aktiv

$\square$ nicht aktiv

6. In welchen medizinischen Bereichen waren Sie bereits tätig ?
$\square \quad$ Onkologie
$\square \quad$ Geriatrie
ㄴ Innere Medizin
$\square$ andere,

7. Jahr der Approbation

8. Tätigkeit in der eigenen Praxis Jahre

9. Art der Praxis
$\square \quad$ Einzelpraxis
$\square$ Gemeinschaftspraxis
$\square$ Praxisgemeinschaft

10 Größe der Praxis

ca. Fälle/Quartal

11.Ort der Praxis (Einwohner)
ㅁ $<10.000$
ㅁ $11-50.000$
$\quad 51-100.000$
ㅁ $>100.000$

Eigene Anmerkungen: 
8.2 Fragebogen (Grundauszählung) 


\section{Abteilung Allgemeinmedizin - Universität Göttingen}

\section{Allgemeinärztliche Einstellungen zur Sterbehilfe}

Teil 1: Fallbeispiele

\section{Anmerkung:}

Bitte beantworten Sie die folgenden Fallbeispiele unter der Annahme, dass sowohl aktive als auch passive Sterbehilfe in Deutschland legal seien.

1. Ein voll zurechnungsfähiger Patient leidet an einem fortgeschrittenem Prostata-Karzinom mit Knochenmetastasen und hat voraussichtlich nur noch wenige Monate zu leben. Es besteht keine Aussicht auf eine Heilung oder eine Verminderung des Krankheitsprogresses. Trotz Ausschöpfung aller palliativen Maßnahmen wie NSAID, Morphin, Hormontherapie, Strahlentherapie, Nervenblockaden etc. verursachen die Metastasen unerträgliche Schmerzen. Sie betreuen den Patienten seit zwei Jahren. Er wurde von einem Psychiater als nicht depressiv beurteilt und bittet Sie nun zum wiederholten Male um Sterbehilfe.

Wie würden Sie handeln?

$\square \quad$ Ich lehne die Durchführung von Sterbehilfe ab und versorge den Patienten mit palliativen Maßnahmen so gut wie möglich.

$\square \quad$ Ich verordne dem Patienten eine große Menge an Morphin und instruiere inn, in welcher Weise die Einnahme zum Tod führt.

$\square \quad$ Ich verzichte auf die weitere Behandlung des Patienten

$\square \quad$ Ich verabreiche dem Patienten eine Injektion mit einer tödlichen Menge eines Medikamentes

$\square \quad$ Keine der angegebenen Möglichkeiten, sondern

2. Ein voll zurechnungsfähiger Patient leidet unter einem fortgeschrittenen Malignom und hat voraussichtlich nur noch wenige Monate zu leben. Es bestehen keine Aussichten auf eine Heilung oder eine Verminderung des Krankheitsprogresses. Unter der jetzigen Therapie ist der Patient schmerzfrei und kann für sich selbst sorgen. Er ist jedoch zunehmend besorgt über die Belastung, die durch die Verschlechterung der Krankheit und den Tod auf seine Familie zukommen wird. Der Patient wurde von einem Psychiater als klinisch nicht depressiv beurteilt, fragt aber zum wiederholten Male nach einer lebensbeendenden Injektion.

Wie würden Sie handeln?

$\square \quad$ Ich lehne die Durchführung von Sterbehilfe ab und versorge den Patienten mit palliativen Maßnahmen so gut wie möglich.

$\square \quad$ Ich verordne dem Patienten eine große Menge an Morphin und instruiere inn, in welcher Weise die Einnahme zum Tod führt.

$\square \quad$ Ich verzichte auf die weitere Behandlung des Patienten

$\square \quad$ Ich verabreiche dem Patienten eine Injektion mit einer tödlichen Menge eines Medikamentes

$\square \quad$ Keine der angegebenen Möglichkeiten, sondern

3. Ein voll zurechnungsfähiger Patient leidet unter einem fortgeschrittenen Malignom und hat voraussichtlich nur noch wenige Monate zu leben. Es bestehen keine Aussichten auf eine Heilung oder eine Verminderung des Krankheitsprogresses. Unter der jetzigen Therapie ist der Patient schmerzfrei. Er kann jedoch weder das Bett verlassen noch sich selbst versorgen. Der Patient wurde von einem Psychiater als klinisch nicht depressiv beurteilt, fragt aber zum wiederholten Male nach einer lebensbeendenden Injektion.

Wie würden Sie handeln?

$\square \quad$ Ich lehne die Durchführung von Sterbehilfe ab und versorge den Patienten mit palliativen Maßnahmen so gut wie möglich.

$\square \quad$ Ich verordne dem Patienten eine große Menge an Morphin und instruiere inn, in welcher Weise die Einnahme zum Tod führt.

$\square \quad$ Ich verzichte auf die weitere Behandlung des Patienten

$\square \quad$ Ich verabreiche dem Patienten eine Injektion mit einer tödlichen Menge eines Medikamentes

$\square \quad$ Keine der angegebenen Möglichkeiten, sondern

4. Ein voll zurechnungsfähiger Patient leidet unter einem fortgeschrittenen Malignom und hat voraussichtlich nur noch wenige Monate zu leben. Es bestehen keine Aussichten auf eine Heilung oder eine Verminderung des Krankheitsprogresses. Unter der jetzigen Therapie ist der Patient schmerzfrei und kann sich selbst versorgen, findet sein Leben aber sinn- und zwecklos. Der Patient wurde von einem Psychiater als klinisch nicht depressiv beurteilt, fragt aber zum wiederholten Male nach einer lebensbeendenden Injektion.

\section{Wie würden Sie handeln?}

$\square \quad$ Ich lehne die Durchführung von Sterbehilfe ab und versorge den Patienten mit palliativen Maßnahmen so gut wie möglich.

$\square \quad$ Ich verordne dem Patienten eine große Menge an Morphin und instruiere inn, in welcher Weise die Einnahme zum Tod führt.

$\square \quad$ Ich verzichte auf die weitere Behandlung des Patienten

$\square \quad$ Ich verabreiche dem Patienten eine Injektion mit einer tödlichen Menge eines Medikamentes 


\section{Teil 2}

1.

Haben Sie selbst oder ein Angehöriger unter einer lebensbedrohlichen Erkrankung gelitten ?

$\square$ ja $n=153(66 \%)$

$\square$ nein $n=79(34 \%)$

2. Haben Sie von Patientenseite schon Nachfragen nach Sterbehilfe erhalten ?
Aktive Sterbehilfe
$\square$ ja
$\mathrm{n}=142(62 \%)$
Passive Sterbehilfe
$\square \quad$ nein
$\mathrm{n}=87(38 \%)$
$\square \quad$ ja
$\mathrm{n}=165(73 \%)$
$n=61(27 \%)$

Wenn ja, wie oft innerhalb der letzten 10 Jahre?

\begin{tabular}{llll} 
Aktive Sterbehilfe & & \multicolumn{2}{c}{ Passive Sterbehilfe } \\
$\square \quad$ Einmal & $\mathbf{n = 3 0 ( 2 2 \% )}$ & $\square$ & Einmal \\
$\square \quad 2-5 \mathrm{mal}$ & $\mathbf{n = 7 1}(\mathbf{5 1} \%)$ & $\square$ & $2-5 \mathrm{mal}$ \\
$\square \quad>5 \mathrm{mal}$ & $\mathbf{n = 3 7}(\mathbf{2 7 \%})$ & $\square$ & $\square$ mal
\end{tabular}

3. Mit welcher der folgenden Vorgehensweisen könnten Sie sich identifizieren, wenn Sie von Patienten um Sterbehilfe gebeten werden würden? (Mehrere Antworten möglich)

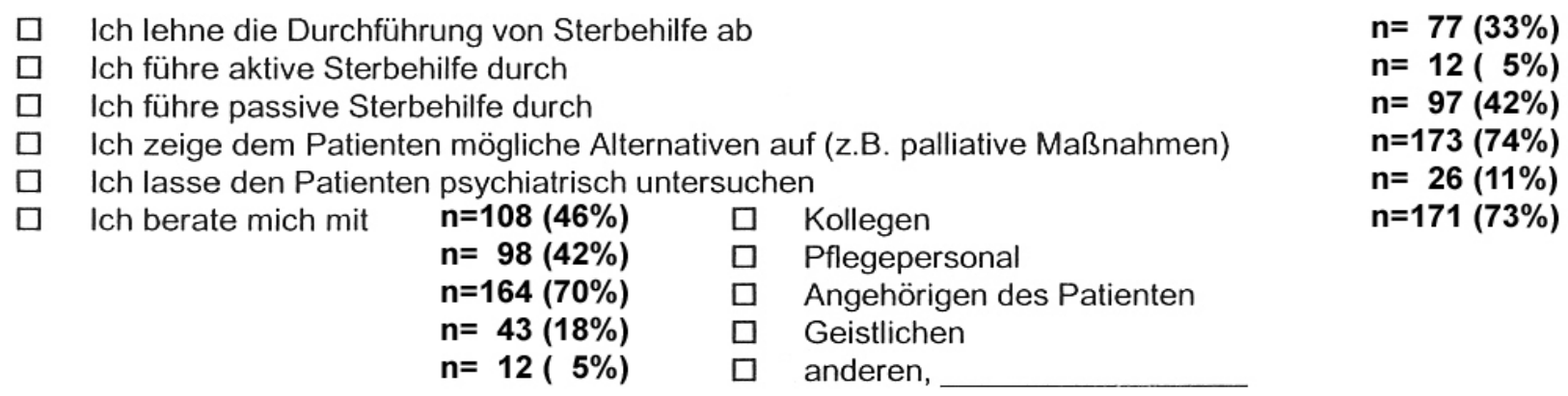

4. Falls Sie jemals Sterbehilfe durchgeführt haben, glauben Sie auch rückblickend, das Richtige getan zu haben?
Aktive Sterbehilfe
$\square \quad$ ja
$\mathrm{n}=\mathbf{2 4}(\mathbf{7 8 \%})$
Passive Sterbehilfe
$\square \quad$ nein
$n=6(19 \%)$
$\square$ ja
$\mathrm{n}=86(97 \%)$
$\square$ unsicher, weil
$n=1(3 \%)$
$\square \quad$ nein
$n=3(3 \%)$
$n=0(0 \%)$

5. Halten Sie Sterbehilfe durch Ärzte für ein akzeptables Vorgehen ?
Aktive Sterbehilfe
Passive Sterbehilfe

$\square \quad$ ja, unter bestimmten Umständen $n=75(35 \%) \square$

ja, unter bestimmten Umständen

$\mathrm{n}=184(80 \%)$

$\square \quad$ nein, unter keinen Umständen $n=141(65 \%)$

nein, unter keinen Umständen

$n=45(20 \%)$

Welche der folgenden Kriterien spielen für diese Entscheidung eine Rolle ? (Mehrere Anworten möglich)

Erlösung des Patienten von Schmerzen

Recht auf einen selbstbestimmten Tod

Ermöglichen eines Sterbens in Würde

Befreiung der Angehörigen von Lasten

Entlastung des Gesundheitssystems

Religiöse Gründe

Eid des Hippokrates

Historische Gründe (“Drittes Reich”)

Juristische Gründe

Ausreichende Palliativmedizin

Mögliche ärztliche Fehleinschätzung

Möglichkeit des Missbrauchs

Andere:

\begin{tabular}{|c|c|}
\hline & Sterbehilfe \\
\hline$\square$ & $n=65(28 \%)$ \\
\hline$\square$ & $n=34(15 \%)$ \\
\hline $\bar{\square}$ & $n=56(24 \%)$ \\
\hline $\bar{\square}$ & $n=4(2 \%)$ \\
\hline$\square$ & $n=2(1 \%)$ \\
\hline $\bar{\square}$ & $n=33(14 \%)$ \\
\hline$\square$ & $n=32(14 \%)$ \\
\hline$\vec{\square}$ & $n=29(12 \%)$ \\
\hline$\square$ & $n=33(14 \%)$ \\
\hline$\square$ & $n=52(22 \%)$ \\
\hline 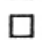 & $n=73(31 \%)$ \\
\hline$\square$ & $n=80(34 \%)$ \\
\hline$\square$ & $n=4(2 \%)$ \\
\hline
\end{tabular}


6. Sofern Sie die Durchführung von Sterbehilfe nicht generell ablehnen: Welche Bedingungen könnten eine solche

Entscheidung für Sie erleichtern? (Mehrere Antworten möglich)

Keine strafrechtliche Verfolgung

Unabhängige Beurteilung durch andere Ärzte

Wiederholter Wunsch des Patienten

Schriftlicher Wunsch des Patienten

Zustimmung der Angehörigen

Ablehnung palliativer Massnahmen durch den Patienten

Ausschluss einer psychischen Erkrankung

Volle Zurechnungsfähigkeit

Begrenzte Lebenserwartung (z.B.<6 Mon.)

Nicht beherrschbare Schmerzen

Gute Kenntnis / Lange Betreuung des Patienten

Mindestalter des Patienten

Andere:

$\begin{array}{ll}\text { Aktive Sterbehilfe } \\ \square & \mathrm{n}=\mathbf{6 4}(\mathbf{2 7} \%) \\ \square & \mathrm{n}=52(22 \%) \\ \square & \mathrm{n}=58(25 \%) \\ \square & \mathrm{n}=52(22 \%) \\ \square & \mathrm{n}=34(15 \%) \\ \square & \mathrm{n}=12(5 \%) \\ \square & \mathrm{n}=32(14 \%) \\ \square & \mathrm{n}=\mathbf{4 4}(19 \%) \\ \square & \mathrm{n}=\mathbf{2 9}(12 \%) \\ \square & \mathrm{n}=68(29 \%) \\ \square & \mathrm{n}=52(22 \%) \\ \square & \mathrm{n}=\mathbf{9}(4 \%) \\ \square & \mathrm{n}=3(1 \%)\end{array}$

Passive Sterbehilfe

$\begin{array}{ll}\square & n=115(49 \%) \\ \square & n=89(38 \%) \\ \square & n=120(52 \%) \\ \square & n=104(45 \%) \\ \square & n=76(33 \%) \\ \square & n=37(16 \%) \\ \square & n=71(30 \%) \\ \square & n=87(37 \%) \\ \square & n=59(25 \%) \\ \square & n=142(61 \%) \\ \square & n=127(55 \%) \\ \square & n=20(9 \%) \\ \square & n=4(2 \%)\end{array}$

7. Besteht für Sie ein moralisch relevanter Unterschied zwischen der Durchführung aktiver Sterbehilfe

(z.B. der Injektion eines tödlichen Medikaments) und passiver Sterbehilfe (z.B. Zurverfügungstellung und Instruktion der Einnahme einer tödlichen Dosis eines Medikaments)?

\begin{tabular}{lll}
$\square$ & ja, und zwar & $\mathbf{n}=112(51 \%)$ \\
\cline { 2 - 3 } & nein & $\mathbf{n}=93(42 \%)$ \\
$\square$ & unsicher, weil & $\mathbf{n}=16(7 \%)$
\end{tabular}

8. Haben Sie jemals Mittel zur Durchführung eines Suizids zur Verfügung gestellt?

\begin{tabular}{lll}
$\square$ & ja & $\mathbf{n}=\mathbf{1 3}(\mathbf{6 \%})$ \\
$\square$ & nein & $\mathbf{n = 2 1 1 ( 9 0 \% )}$ \\
$\square$ & unsicher, weil & $\mathbf{n}=\mathbf{9}(\mathbf{4 \%})$ \\
\hline
\end{tabular}

9. Halten Sie in Deutschland eine gesetzliche Regelung zur Sterbehilfe für erforderlich?

\begin{tabular}{|c|c|c|c|c|}
\hline \multicolumn{2}{|l|}{ Aktive Sterbehilfe } & \multicolumn{3}{|c|}{ Passive Sterbehilfe } \\
\hline$\square \quad$ ja & $n=105(50 \%)$ & $\square$ & ja & $n=131(59 \%)$ \\
\hline nein & $n=98(46 \%)$ & $\square$ & nein & $n=82(37 \%)$ \\
\hline unsicher, weil & $n=9(4 \%)$ & $\square$ & unsicher, weil & $n=10(4 \%)$ \\
\hline
\end{tabular}

10. Wären Sie bereit, Sterbehilfe durchzuführen, wenn dafür in Deutschland eine gesetzliche Grundlage bestünde ?

Aktive Sterbehilfe

$\begin{array}{lll}\square & \text { ja } & n=38(18 \%) \\ \square & \text { nein } & n=141(68 \%) \\ \square & \text { unsicher, weil } & n=29(14 \%)\end{array}$

\section{Passive Sterbehilfe}

\begin{tabular}{lll}
$\square$ & ja & $n=130(59 \%)$ \\
$\square$ & nein & $n=64(29 \%)$ \\
$\square$ & unsicher, weil & $n=27(12 \%)$ \\
\hline
\end{tabular}

11. Es existieren unterschiedliche Modelle zur Sterbehilfe. Welches der folgenden Modelle würden Sie favorisieren ?

In den Niederlanden wurde die aktive Sterbehilfe legalisiert. Um als Arzt Sterbehilfe durchzuführen, müssen bestimmte Voraussetzungen erfüllt sein. Die wichtigsten Punkte lauten: Der Patient muss mehrfach um Sterbehilfe gebeten haben, an einer tödlichen Krankheit mit infauster Prognose leiden und über Alternativen wie Palliativmedizin informiert worden sein. Schließlich muss vor der Tötung ein weiterer Arzt hinzugezogen werden. Die Entscheidung des Arztes, Sterbehilfe durchzuführen, wird von einem unabhängigen Gremium überprüft.

I In der Schweiz besteht für voll zurechnungsfähige Personen die Möglichkeit, als Mitglied einer Organisation Hilfe bei der Sterbevorbereitung, der Sterbegleitung sowie der Durchführung von Patientenverfügungen gegenüber Ärzten, Kliniken, Alters- und Pflegeheimen zu erhalten. Gegen einen Mitgliedsbeitrag hilft diese Organisation durch" personelle, finanzielle und juristische Möglichkeiten” bei "ärztlich diagnostizierten hoffnungslosen oder unheilbaren Krankheiten, unerträglichen Schmerzen oder unzumutbare Behinderungen“.

In Deutschland ist die aktive Sterbehilfe sowie das Töten auf Verlangen strafbar. Die Nichtaufnahme oder Beendigung einer Intensivbehandlung ist nur dann nicht strafbar, wenn sie aus einer frei verantwortlichen Willensentscheidung des Patienten (z.B. Patientenverfügung) entstanden ist. Unter bestimmten Voraussetzungen ist der Abbruch lebenserhaltender Massnahmen zulässig, wenn dies dem "mutmasslichen" Willen des Patienten entspricht. $\mathbf{n = 1 0 2}$ (50\%)

12. Fühlten Sie sich während Ihrer Ausbildung zum Arzt ausreichend mit den Themen Sterben und Sterbehilfe konfrontiert ? $\begin{array}{lll}\square & \text { ja } & \mathbf{n}=\mathbf{2 6}(\mathbf{1 1 \%} \%) \\ \square & \text { nein } & \mathbf{n}=\mathbf{2 0 3}(\mathbf{8 9 \% )}\end{array}$

13. Halten Sie einen Vergleich zwischen der Euthanasie im sog. "Dritten Reich" und der Durchführung von Sterbehilfe heutzutage für gerechtfertigt?
$\square \quad$ ja, weil
$\square \quad$ nein, weil $n=48(21 \%)$ $\mathrm{n}=182(79 \%)$ Bitte wenden 


\section{Teil 3: Persönliche Angaben}

1. Alter

2. Geschlecht

3. Familienstand

4. Kinder

5. Religion

Religionsausübung
Jahre

weiblich

$\square$ männlich
Durchschnitt: 51 Jahre (Range 29-77)

$n=157(68 \%)$

$n=75(32 \%)$ $\square \quad$ ledig

ㅁ verheiratet /eheähnl. Gemeinschaft

$\square$ feste Partnerschaft

$\square$ geschieden / getrennt lebend

ㅁ verwitwet $n=17(7 \%)$

$\mathrm{n}=182(80 \%)$

$n=8(4 \%)$

$n=16(7 \%)$

$n=5(2 \%)$ $\square \quad$ ja

$\square \quad$ nein

$\square$ evangelisch

ㅁ katholisch

$\square$ andere,

$\square$ keine

aktiv

$\square \quad$ gelegentlich aktiv

$\square$ nicht aktiv $\mathrm{n}=195(84 \%)$

$n=37(16 \%)$

$n=89(38 \%)$

$n=76(33 \%)$

$n=8(4 \%)$

$n=59(25 \%)$

$\mathrm{n}=\mathbf{3 8}(\mathbf{2 0 \% )}$

$n=64(33 \%)$

$\mathrm{n}=91(47 \%)$

6. In welchen medizinischen Bereichen waren Sie bereits tätig ?

\begin{tabular}{lllll}
$\mathbf{n}=\mathbf{7 0}(\mathbf{3 1 \% )}$ & $\square$ & Onkologie & $\square$ & Innere Medizin \\
$\mathbf{n = 9 4}(\mathbf{4 2 \% )}$ & $\square$ & Geriatrie & $\square$ & Psychiatrie \\
$\mathbf{n = 1 2 4}(\mathbf{5 5 \% )}$ & $\square$ & andere, & & \\
\hline
\end{tabular}

7. Jahr der Approbation

8. Tätigkeit in der eigenen Praxis Jahre

9. Art der Praxis

10 Größe der Praxis ca. Fälle/Quartal

$\square$ Gemeinschaftspraxis

$\square$ Praxisgemeinschaft

- Fäle/Quartal

ab $1983: n=63(28 \%)$

1965-1982: $n=139(62 \%)$

vor $1965: n=24(11 \%)$

bis 10 Jahre : $\mathrm{n}=87(38 \%)$

11-20 Jahre $\quad n=82(36 \%)$

mehr als 21 Jahre: $n=60(26 \%)$

$$
\begin{aligned}
& n=171(75 \%) \\
& n=43(19 \%) \\
& n=15(6 \%)
\end{aligned}
$$

$<=750 \quad: n=45(20 \%)$

$751-1500: n=145(64 \%)$

1501-2250: $n=28(12 \%)$

2251-3000: $n=8(4 \%)$

$\begin{array}{rllll}\text { 11.Ort der Praxis (Einwohner)n=85(37\%) } \square & <10.000 & \square & 11-50.000 & \mathbf{n = 8 9}(\mathbf{3 8 \%}) \\ \mathbf{n}=\mathbf{1 8}(\mathbf{8} \%) \square & 51-100.000 & \square>100.000 & \mathbf{n = 4 0 ( 1 7 \% )}\end{array}$

$\begin{array}{rllll}\text { 11.Ort der Praxis (Einwohner)n=85(37\%) } \square & <10.000 & \square & 11-50.000 & \mathbf{n = 8 9}(\mathbf{3 8 \%}) \\ \mathbf{n}=\mathbf{1 8}(\mathbf{8} \%) \square & 51-100.000 & \square>100.000 & \mathbf{n = 4 0 ( 1 7 \% )}\end{array}$

Eigene Anmerkungen: 


\subsection{Freie Anmerkungen}

-„Sterbehilfe darf nicht gesetzlich geregelt werde. Die Erfahrungen werden dann zeigen, dass die Gesellschaft (Kassen, Angehörige) Druck auf den Patienten ausüben, um von seinem Recht auf Sterbehilfe gebrauch zu machen. Sterbehilfe muss für den Arzt immer eine Einzelfallentscheidung am Rande der Illegalität sein, sonst wird sie bürokratisiert.“

-„Sterbebegleitung im Rahmen der hausärztlichen Tätigkeit erlebe ich immer wieder als eine besonders fordernde, teilweise belastende, aber auch intensive Begegnung und die eigene Reifung fördernde Tätigkeit. Schwierig wird es für mich oft, die notwendige Zeit für die eigene Trauerarbeit zu finden. Hilfreich ist hier immer wieder die Balintgruppe“

-„Aktive und passive Sterbehilfe darf nur in sehr seltenen Fällen in Frage kommen, aber wenn doch, halte ich die aktive Form für humaner und ehrlicher."

-„Als Hausarzt halte ich aktive und passive Sterbehilfe für untragbar; der Patient sollte sich in jeder Situation sicher fühlen, nicht von seinem Arzt umgebracht zu werden. Eine aktive Sterbehilfe im Todesprozess zur Abkürzung des Todeskampfes halte ich für angebracht und menschlich."

-„Als Ärztin für Homöopathie habe ich in der Palliation noch zusätzliche Möglichkeiten. Ich strebe mit allen Mitteln ein friedliches Sterben an, auch auf Kosten meiner Zeit. Daher war es für mich nicht erforderlich passive Sterbehilfe durchzuführen."

-„Die Anlage von PEG durch Hochbetagte zur künstlichen Lebensverlängerung mit der Folge von Ulcera wegen Bewegungsunfähigkeit ist ein Skandal. Warum muss ein 90jähriger am Leben gehalten werden, wenn er bewusstlos vor sich hin vegetiert?"

-„Die Frage Sterbehilfe ja oder nein ist eine Frage einer sehr persönlichen Verantwortung und Beziehung zum Betroffenen. Die Entscheidung kann - unter welchem Modell auch immer- nur sehr persönlich und nie instrumentalisiert sein."

-„Die juristische Überfrachtung der Medizin hat dazu geführt, dass sinnlose Maßnahmen aus Angst vor dem Staatsanwalt fortgeführt werden. Die moderne Medizin scheint immer weniger zu akzeptieren, dass Tod und Sterben natürliche Vorgänge sind, die ohne Technik und Labor ablaufen. PEG und Infusionen sind nicht immer human!".

-„Dieses Thema wurde erst aktuell, als es um das Sparen im Gesundheitssystem ging. Sozial verträgliches Ableben?"

-„Durch die Euthanasie im „Dritten Reich“ werden wir in Deutschland noch viele Jahre über Sterbehilfe diskutieren. Ich favorisiere das Patiententestament, welches den Umfang der medizini- 
schen Maßnahmen in der gegebenen Situation genau abgrenzt. Beim Aufsetzen bin ich den Patienten auf Bitte behilflich."

-„Effektive palliative und psychotherapeutische Behandlung für einen Patienten muss möglich sein. Meine Patienten haben Angst vor der Verlängerung ihres Leidens. Sie sollen sicher sein, dass ihr Arzt sie unter keinem - gleich wie geartetem - Grund umbringt. Wir sollten uns nicht von dem Druck der Medien und dem emotionalen Druck des Patienten auf eventuell zukünftiges Verhalten festlegen."

-„Ein Platz im Hospiz unserer Stadt kostet 12.000DM/Monat. Gut, dass so viele Bürger dafür spenden."

-„Eine legale Sterbehilfe sollte nicht in der Verantwortung des Arztes liegen dürfen. Die Entscheidung zur Sterbehilfe und deren Durchführung muss -um Missbrauch vorzubeugen- notariell o.ä. von individuell zusammengesetzten Gremien (Angehörige, Richter, Ärzte, Pfarrer) getroffen werden."

-„Eine wirklich umfassende psychische und soziale Betreuung, in der der Patient sich in jeder Situation geborgen fühlt, würde m.E. nach die ganze Problematik wesentlich herabschrauben. Leider bestehen in dieser Richtung große Lücken.“

-„Es ist höchste Zeit für diese Diskussion!“

-„Es wird allerhöchste Zeit für diese Diskussion. Es kann nicht sein, dass erst Politiker in eine solche Situation kommen müssen- siehe Brandenburg.“

-„Gesetzliche Regelung von Sterbehilfe ist nicht nötig und auch nicht zu wünschen. Sterbehilfe ist und soll ein intimes und vertrauensvolles Verhältnis zwischen Arzt, dem Patienten und evtl. dessen Angehörigen bleiben. Gesetzliche Regelungen können Alten und Kranken das Gefühl vermitteln, die Gesellschaft wünsche ihr Ableben."

-„Eine gesetzliche Regelung ist überflüssig, da sie meine Entscheidung nicht beeinflusst. Diese ist abhängig von der Kenntnis des Patienten, seinem Krankheitsbild, seinem Wunsch und seinem Umfeld. Die Gefahr einer gesetzlichen Regelung: Alte Menschen könnten das Gefühl bekommen abtreten zu sollen."

-,ICh bilde mich in den letzten Jahren zunehmend fort in Palliativmedizin, Schmerztherapie und Sterbebegleitung und erachte es als wichtigen Teil der ärztlichen Berufsausübung!"

-„ICh habe auch als Arzt kein Recht in das Schicksal, die Vorsehung von Gott in bestimmte Abläufe - oder wie Sie es immer nennen mögen - einzugreifen und nehme dadurch Anteil am Leid, das zu ertragen ist." 
-„In diesem Konflikt habe ich schon Morphium s.c. gespritzt - immer nur Tage oder Stunden vor dem sowieso zu erwartetem Tod. Aber gut ging’s mir dabei auch nicht!“”

- „Ich halte ein selbstbestimmtes Leben und Sterben in Würde für bedeutsam.“

-„Ich persönlich habe Sterbehilfe für mich befürwortet, denn ich möchte nicht elendiglich zu Grunde gehen und meine Familie soll nicht wegen mir leiden. Macht endlich Schluss mit der Vergangenheit! Blickt endlich positiv in die Zukunft!“

-,Ich rege zu Vorsorgevollmachten und Patientenverfügungen an. Den Sterbewunsch sehe ich vor dem Hintergrund des keinen Sinn mehr im Leben sehen und der schwindenden Geborgenheit im Bezugssystem (Familie, Religion etc.), das ich zu stärken versuche. Sterben wie auch Leben (ich betreue ein Schwerst-/Mehrfachbehindertenheim, die Schwiegermutter ist ein apallischer Schwerstfall) macht aber Sinn und nicht nur Schmerz."

- „ICh würde mir eine gesetzliche Entlastung aller Therapeuten dahingehend wünschen, dass das Unterlassen sinnloser und damit häufig nichtsbringender Therapien als seriös und verantwortlich rechtlich gewertet wird."

-„Im zur Zeit geltenden Gesundheitssystem mit Solidarzwang und minimalster Eigenverantwortung und Protektion von Kassen, Ärztefunktionären und alteingesessenen Ärzten kann in Anbetracht des bevorstehenden Systemkollapses mit fehlenden ethischen und moralischen Werten in unserer Gesellschaft nur eindringlich vor Sterbehilfe durch Ärzte gewarnt werden."

-„Jedes Sterben ist anders. Der Prozess ist wie eine Geburt und braucht dringend mehr kompetente Helfer und Begleiter für die Angehörigen und Sterbenden."

-„Regelmäßige Anwendung von aktiver oder passiver Sterbehilfe senkt die Hemmschwelle und könnte damit den Übergang zur stattgehabten Euthanasie unscharf werden lassen.“

-„Sehr wichtig für mich ist der langjährige Kontakt zum Patienten durch den Hausarzt, der eine fundierte Ausbildung, wenigstens religiöse Grundkenntnisse hat und auch während seiner Weiterbildung Gelegenheit hatte, sich psychologisch und praktisch mit Sterbenden zu befassen."

-„Sehr wichtig wäre mir in diesem Zusammenhang eine gute auf Hintergrundwissen basierende Arzt- Patienten Beziehung. Nicht abhängig von Jahren, aber gutem Einschätzungsvermögen der langfristigen Gesamtsituation."

-„Sterbehilfe sollte nicht generell Aufgabe des Arztes sein. M.E. ist eine spezielle Ausbildung und entsprechende religiöse Einstellung nötig.“ 
-„Sterbehilfe kann und darf nur in ganz wenigen und hoffnungslosen Fällen erfolgen - sie darf nie zwingend sein. “

-„Vielleicht ein vermessenes Ziel: Hausärztlicher Versuch nicht erst Sterbenden, sondern vorher schon bei der Suche nach Sinngebung des Lebens bis hin zum Sterben behilflich zu sein. Frühzeitige und großzügige Schmerztherapie unter Einsatz aller verfügbaren Möglichkeiten!“

-,Wenn ich meinem Patienten zugesichert habe, ihm beim Sterben wenn nötig auch aktiv zu helfen ist es nie dazu gekommen, da der Patient Sicherheit verspürt und die Leiden erträglicher sind.“ 


\section{Literaturverzeichnis}

Abholz HH (1998): Palliativmedizinische Versorgung- eine hausärztliche Aufgabe? Z Allg Med $\underline{74}$, 957-961

Baader G, Schultz U: Medizin und Nationalsozialismus. Tabuisierte Vergangenheit- Ungebrochene Tradition? Dokumentation des Gesundheitstages Berlin 1980. Mabuse, Frankfurt/M. 1980

Bachmann JG, Alcser KH, Doukas DJ, Lichtenstein RL, Corning AD, Brody H (1996): Attitudes of Michigan physicians and the public toward legalizing physician- assisted suicide and voluntary euthanasia. N Engl J Med 334, 303-309

Back AL, Wallace JI, Starks HE, Pearlman RA (1996): Physician- assisted suicide and euthanasia in Washington state. J Am Med Assoc 275, 919-925

Baume P, O' Malley E (1994): Euthanasia: attitudes and practices of medical practitioners. Med J Aust $\underline{161}, 137-144$

Baume P, O' Malley E (1996): Alberta Euthanasia Survey: 3-year follow up. Can Med Assoc J $\underline{155}$, 885-890

Beauchamp TL, Davidson AL (1979): The definition of euthanasia. J Med Philos $\underline{4}, 294-312$

Brody H: Assisted suicide (1993): A challenge for family physicians. J Fam Pract $\underline{37,123-125}$

Bundesärztekammer (1998): Grundsätze der Bundesärztekammer zur ärztlichen Sterbebegleitung. Dtsch Ärztebl $\underline{95}$, A-2366-2367

Bundesärztekammer (1999): Handreichung für Ärzte zum Umgang mit Patientenverfügungen. Dtsch Ärztebl $\underline{96}$, A-2720-2721

Cohen JS, Fihn SD, Boyko EJ, Jonsen AR, Wood RW (1994): Attitudes toward assisted suicide and euthanasia among physicians in Washington state. N Engl J Med 331, 89-94

Council of the Royal College of Physicians (2001): Medical treatment at the end of life. A position statement. Clin Med 1, 115-117

Csef H, Heindl B (1998): Einstellungen zur Sterbehilfe bei deutschen Ärzten. Eine repräsentative Befragung im ärztlichen Kreisverband Würzburg. Dtsch Med Wochenschr $\underline{123}$, 1501-1506

Dickinson GE, Lancaster CJ, Sumner ED, Cohen JS (1996): Attitudes toward assisted suicide and euthanasia among physicians in South Carolina. J S C Med Assoc 92, 395- 399 
Dornberg M: Behandlungsverzicht, Behandlungsabbruch und Sterbehilfe aus der Sicht internistischer Krankenhausärzte. Ergebnisse einer Befragung und medizinethische Bewertung. Med. Diss. Freiburg 1997

Duberstein PR, Conwell Y, Cox C, Podgorski CA, Glazer RS, Caine ED (1995): Attitudes toward self-determined death: A survey of primary care physicians. J Am Geriatr Soc $\underline{43}, 395-400$

Emanuel EJ, Fairclough DL, Daniels ER, Clarridge BR (1996): Euthanasia and physician assisted suicide: attitudes and experiences of oncology patients, oncologists, and the public. Lancet $\underline{347}$, 1805-1810

Folker AP, Holtug N, Jensen AB, Kappel K, Nielsen JK, Norup M (1996): Experiences and attitudes towards end-of-life decisions amongst Danish physicians. Bioethics $\underline{10}, 233-249$

Forde R, Gjerlow A, Falkum E (1997): The ethics of euthanasia- attitudes and practice among Norwegian physicians. Soc Sci Med $\underline{45}, 887-892$

Fried TR, Stein MD, O' Sullivan PS (1993): Limits of patient autonomy. Physician attitudes and practices regarding life-sustaining treatments and euthanasia. Arch Intern Med $\underline{153}, 722-728$

Gahl K (2002): Ärztliche Indikation zum Töten? Dtsch Med Wochenschr 127, 866-869

Gassmann R, Schnabel E: Die Betreuung Sterbender durch den Hausarzt. Med. Diss. Berlin 1996

Hegselmann R, Merkel R: Zur Debatte über Euthanasie. Suhrkamp, Frankfurt/M. 1991

Helou A, Wende A, Hecke T, Rohrmann S, Buser K, Dierks ML (2000): Das öffentliche Meinungsbild zur aktiven Sterbehilfe. Ergebnisse eines Pilotprojekts. Dtsch Med Wochenschr $\underline{125}$, 308-315

Keane WG, Gould JH, Millard PH (1983): Death in practice. J R Coll Gen Pract 33, 347-351

Kinsella TD, Verhoef MJ (1993 a): Alberta Euthanasia Survey: 1. Physicians' opinions about the morality and legalization of active euthanasia. Can Med Assoc J $\underline{148}, 1921-1926$

Kinsella TD, Verhoef MJ (1993 b): Alberta Euthanasia Survey: 2. Physicians' opinions about the acceptance of active euthanasia as a medical act and the reporting of such practice. Can Med Assoc J 148, 1929-1933

Kinsella TD, Verhoef MJ (1995): Assisted suicide: opinions of Alberta physicians. Clin Invest Med $\underline{18}, 406-412$

Kinsella TD, Verhoef MJ (1996): Alberta Euthanasia Survey: 3-year follow up. Can Med Assoc J $\underline{155}, 885-890$ 
Kirschner R, Elkeles T (1998): Ärztliche Handlungsmuster und Einstellungen zur Sterbehilfe in Deutschland. Eine Repräsentativbefragung unter Ärzten. Gesundheitswesen $\underline{60}$, 247-253

Klaschik E (1999): Sterbehilfe -Sterbebegleitung. Internist $\underline{40}, 276-282$

Klaschik E, Ostgathe C, Nauck F (2002): Palliativmedizin. Defizite in der studentischen Ausbildung. Dtsch Ärztebl $\underline{99}$, A 1286-1288

Kuhse H, Singer P (1988): Doctors' practices and attitudes regarding voluntary euthanasia. Med J Aust $\underline{148}, 623-627$

Lee MA, Nelson HD, Tilden VP, Ganzini L, Schmidt TA, Tolle SW (1996): Legalizing assisted suicide- views of physicians in Oregon. N Engl J Med 334, 310-315

Lichtenstein RL, Alcser KH, Corning AD, Bachmann JG, Doukas DJ (1997): Black and white attitudes towards physician-assisted suicide. J Natl Med Assoc 모, 125-133

Mc Glade KJ, Louise S, Bunting BP, Gallagher AG (2000): Voluntary euthanasia in Northern Ireland: general practitioners' beliefs, experiences, and actions. Br J Gen Pract $\underline{50}, 794-797$

Meier DE, Emmons CA, Wallenstein S, Quill T, Morrison RS, Cassel CK (1998): A national survey of physician- assisted suicide and euthanasia in the United States. N Engl J Med $\underline{338}, 1193-1201$

Miller GF, Quill TE, Brody H, Fletcher JC, Gostin LO, Meier DE (1994): Regulating physicianassisted death. N Engl J Med $\underline{331}, 119-122$

Molloy DW, Guyatt GH, Alemayehu E, Mc Ilroy W, Willan A, Eisemann M, Abraham G, Basile J, Penington G, Mc Murdo MET (1991): Factors affecting physicians' decisions on caring for an incompetent elderly patient: an international study. Can Med Assoc J $\underline{145}, 947-952$

Müller- Busch HC, Klaschik E, Odunco F, Schindler T, Woskanjan S (2003): Euthanasie bei unerträglichem Leid? Eine Studie der Deutschen Gesellschaft für Palliativmedizin zum Thema Sterbehilfe im Jahre 2002. Z Palliativ Med $\underline{4}, 75-84$

Niethammer D: Menschenwürdig sterben aus der Sicht eines Arztes; in: Menschenwürdig sterben; hrsg. v. Jens W, Kühn H u.a.; Piper, München 1995, 332-334

Onwuteaka- Philipsen BD, Muller MT, van der Wal G, van Eijk JTM, Ribbe MW (1995): Attitudes of Dutch general practitioners and nursing home physicians to active voluntary euthanasia and physician- assisted suicide. Arch Fam Med 4 , 951-955 
Oorschot B, Schweitzer S (2003): Ambulante Versorgung von Tumorpatienten im finalen Stadium. Ergebnisse einer schriftlichen Hausärztebefragung in Thüringen. Dtsch Med Wochenschr $\underline{128}$, 2295-2299

Peretti-Watel P, Bendiane MK, Pegliasco H, Lapiana JM, Favre R, Galinier A, Moatti JP (2003): Doctors' opinions on euthanasia, end of life care, and doctor-patient communication: telephon survey in France. BMJ $\underline{327}$, 595-596

Quante M (1998): Passive, indirekte und direkte aktive Sterbehilfe- deskriptiv und ethisch tragfähige Entscheidungen? Ethik Med 10, 206-226

Quill TE, Cassel C (2003): Professional organizations' position statements on physician-assisted suicide: a case for studied neutrality. Ann Intern Med $\underline{138}, 208-211$

Radulovic S, Mojsilovic S (1998): Attitudes of oncologists, family doctors, medical students and lawyers to euthanasia. Support Care Cancer $\underline{6}, 410-415$

Schindler T, Woskanjan S, Abholz HH (2002): Palliativmedizinischer Konsiliardienst für Berliner Hausärzte- quantifizierbare Ergebnisse und nichtquantifizierbare Erfahrungen nach zweijähriger Tätigkeit. Z Allg Med $\underline{78}, 75-81$

Shapiro RS, Derse AR, Gottlieb M, Schiedermayer D, Olson M (1994): Willingness to perform euthanasia. A survey of physician attitudes. Arch Intern Med 154, 575-584

Siaw LK, Tan SY (1996): How Hawaii' s doctors feel about physician-assisted suicide and euthanasia: an overview. Hawaii Med J $\underline{55}$, 296-298

Singh BK (1979): Correlates of attitudes towards euthanasia. Soc Biol $\underline{26}$, 247-254

Slome LR, Mitchell TF, Charlebois E (1997): Physician assisted suicide and patients with human immunodeficiency virus disease. N Engl J Med $\underline{336}, 417-421$

Sohn W (2002): Sterbehilfe: Regelungsbedarf für ungeklärte Fragen. Z Allg Med $\underline{78}, 63-67$

Steinberg MA, Najman JM, Cartwright CM, MacDonald SM, Williams GM (1997): End-of-life decision-making: community and medical practitioners' perspective. Med J Aust 166, 131-134

Stevens CA, Hassan R (1994): Management of death, dying and euthanasia: attitudes and practices of medical practitioners in South Australia. J Med Ethics $\underline{20}, 41-46$

Taupitz J (2001): Selbstbestimmtes Sterben. Die Macht der Gesetze ist beschränkt. Dtsch Ärztebl $\underline{98}$, A- 2937-2942 
Van der Maas PJ, van Delden JJM, Pijnenborg L, Looman CW (1991): Euthanasia and other medical decisions concerning the end of life. Lancet $\underline{338}, 669-674$

Van der Waal G, van Eijk JT, Leenen HJ, Spreeuwenberg C (1992): Euthanasia and assisted suicide. How often is it practised by family doctors in the Netherlands? Fam Pract $\underline{9}, 130$

Verhoef MJ, Kinsella D (1996): Alberta Euthanasia survey: 3-year follow up. Can Med Assoc J $\underline{155}$, 885-890

Verhoef MJ, van der Wal G (1997): Euthanasia in family practice in the Netherlands. Toward a better understanding. Can Fam Physician $\underline{43}, 231-237$

Waddell C, Clarnette RM, Smith M, Oldham L, Kellehear A (1996): Treatment decision-making at the end of life: a survey of Australian doctors' attitudes towards patients' wishes and euthanasia. Med J Aust $\underline{165}$, 540-544

Ward BJ, Tate PA (1994): Attitudes among NHS doctors to requests for euthanasia. BMJ $\underline{308}, 1332-1334$

Ward RA (1980): Age and acceptance of euthanasia. J Gerontol $\underline{35}, 421-431$

Weber M, Stiehl M, Reiter J, Rittner C (2001): Sorgsames Abwägen der jeweiligen Situation. Ergebnisse einer Ärztebefragung in Rheinland-Pfalz. Dtsch Ärztebl 98, A 3184-3188

Wehkamp KH: Therapieverzicht -Dilemmata medizinischen Entscheidens; in: Sterbehilfe- Handeln oder Unterlassen? Referate einer medizinethischen Fortbildungsveranstaltung vom Zentrum für Ethik und Recht in der Medizin und dem Zentrum für Geriatrie und Gerontologie Freiburg am 19. und 20. Januar 1996; hrsg. v. Illhardt FJ, Heiss HW, Dornberg M u.a.; Schattauer, Stuttgart 1997, 25-33

Wienke A (2000): Deutsche Gesellschaft für Medizinrecht: Der Wille des Menschen zwischen Leben und Sterben. Ausgewählte medizinrechtliche Aspekte des Arzt- Patienten Verhältnisses am Ende des Lebens. Hess Ärztebl 11, 479-480

Wilson I, Kay B, Steven I (1997): General practitioners and euthanasia. Strong opinions and unexpected views. Aust Fam Phys $\underline{26}$, 399-401 


\section{Danksagung}

Ich danke Herrn Prof. Dr. Kochen, MPH, FRCGP, für die freundliche Überlassung des Themas der Dissertation.

Herrn Dr. Maitra danke ich für die engagierte und kontinuierliche Betreuung meiner Arbeit sowie Frau Dr. Becker für ihre Unterstützung und konstruktive Kritik.

Mein besonderer Dank gilt ferner den Ärztinnen und Ärzten, die an dieser Studie mitgewirkt haben. 


\section{Lebenslauf}

Am 28.05.1979 wurde ich in Rüsselsheim als zweites Kind meiner Eltern Wilfried und Gertrud Harfst, geb. Behles geboren. Ich habe zwei Brüder, Daniel und Simon.

1985 bis 1989 besuchte ich die Grundschule in Mainz- Laubenheim. Anschließend besuchte ich das Rabanus Maurus Gymnasium in Mainz, wo ich am 23.06.1998 die allgemeine Hochschulreife erwarb.

Seit dem Wintersemester 1998 studiere ich Humanmedizin an der Universität Göttingen. Nach bestandener ärztlichen Vorpüfung am 14.03.2001 und bestandenem ersten Abschnitt der Ärztlichen Prüfung am 11.04.2002 befinde ich mich zur Zeit im 10. Fachsemester und beabsichtige, den zweiten Abschnitt der Ärztlichen Prüfung im Frühjahr 2004, den dritten Abschnitt der Ärztlichen Prüfung entsprechend ein Jahr später zu absolvieren. 
